TAO, Vol. 11, No. 1, 53-114, March 2000

\title{
The GPS Radio Occultation Technique
}

\author{
E. Robert Kursinski ${ }^{1, \star}$, George A. Hajj ${ }^{1}$, Stephen S. Leroy ${ }^{1}$ and Benjamin Herman ${ }^{2}$
}

(Manuscript received 8 January 2000, in final form 20 February 2000)

\begin{abstract}
Radio occultation observations represent a planetary-scale optics experiment in which the atmosphere acts as a lens and alters the propagation velocity and paths of microwave signals passing through it. In this paper we review the process of inverting the radio occultation observations acquired using the Global Positioning System (GPS) in order to derive atmospheric quantities of interest including temperature, geopotential and water vapor. Beginning with geometric optics, we derive the Abel integral used to transform the observations into profiles of refractivity. In the process, we characterize why the Abel transform works so well as a first approximation for deriving atmospheric profiles. We discuss the resolution of the observations and the improvements that can be achieved via the backpropagation concept where the receiver's position is effectively moved closer to the limb of the Earth in post-processing. We discuss several factors that complicate the observations in the Earth's troposphere including critical refraction and atmospheric multipath. Critical refraction refers to the situation where the bending becomes so great that the occulted signal disappears whereas atmospheric multipath refers to the situation where multiple signal paths connect the transmitter and receiver. We describe the derivation of temperature, pressure and water vapor from the observations including the optimal combining of the occultation observations with weather and climate analyses. We describe some key issues in deriving profiles from real data including the correction of clock errors and ionospheric effects, and the estimation of the resolution and atmospheric Doppler in a self-consistent manner. We conclude by summarizing the expected and achieved accuracy and resolution.
\end{abstract}

(Key words: Atmospheric remote sensing, Radio Occultation, Data assimilation, Temperature, Pressure, Geopotential height, Water vapor, GPS, Climate )

\footnotetext{
${ }^{1}$ Jet Propulsion Laboratory, California Institute of Technology, Pasadena, CA, USA

${ }^{2}$ Department of Atmospheric Sciences, Univ. of Arizona, Tuscon, AZ, USA

* Corresponding author address: Dr. E. Robert Kursinski, Jet Propulsion Laboratory, California Institute of Technology, Pasadena, CA 91109-8099, USA; E-mail: rob.kursinski@jpl.nasa.gov
} 


\section{INTRODUCTION}

Occultation has been useful as a probe of planetary atmospheres for many years. Initially, in stellar occultations, stars have been observed from Earth as they passed behind a planet, the intensity fading as starlight was spread by the increasing thickness of the atmosphere with depth. The fading tendency could be used to approximate the scale height of the atmosphere, but little more. With the advent of interplanetary probes came radio occultation, wherein a radio signal generated on board a spacecraft is tracked at Earth and precise information on the amplitude and phase of the radio signal is retained. Radio occultation data was first inverted to approximate the density of atmospheres by assuming the occulted atmosphere simply retarded the radio signal. With a seminal paper in 1972, Lindal showed that if the bending of the radio beam is measured, that information could be analytically inverted to give the index of refraction as a function of height in the occulted atmosphere with unexpectedly high vertical resolution. This technique was applied to the atmospheres of the outer planets using data from the two Voyager spacecraft and to the atmosphere of Venus using Magellan data giving us perhaps the best existing data on the temperature structure of those atmospheres.

GPS occultation observations offer several important and unique features complementary to other methods of observing Earth's atmosphere. Occultation observations are inherently self-calibrating. GPS occultation observations are made at $\sim 20 \mathrm{~cm}$ wavelengths which can routinely probe to the surface in a limb viewing geometry. The observations provide relatively high vertical resolution of $\sim 1 \mathrm{~km}$ or better. The dynamically important geopotential height of the pressure field is derived directly. Furthermore, the tangent height associated with a given raypath is determined in the retrieval process to an accuracy of order 10 meters even when raypaths are displaced vertically by $50 \mathrm{~km}$ or more relative to paths in the absence of the atmosphere, a capability which is inconceivable with passive limb sounders. Together these features enable a unique limb sounding of the troposphere to the surface under any and all weather conditions providing the first truly global characterization of Earth's atmosphere at $\sim 1 \mathrm{~km}$ or better vertical resolution.

With GPS/MET came the first implementation of the radio occultation technique applied to Earth's atmosphere. It obtained several thousand occultation profiles of the atmosphere, and suddenly there came a demand to develop a system which can automatedly process the measurements to obtain information on atmospheric temperatures and pressures. Various steps are involved in such an automated system, including (1) the acquisition of ancillary data from Earth-based GPS receiver stations, (2) the calculation of transmitter and receiver satellite trajectories with very high precision, (3) the solution of the geometry of the beampath during the occultation, (4) the inversion to retrieve refractive index, and finally (5) the retrieval of customary atmospheric parameters. The first two steps are not covered in this paper.

In this paper we address steps (3) through (5), giving theoretical underpinnings, methodological implementation, and a characterization of the final products. We first give a theoretical basis for the occultation problem by showing how the atmosphere bends a radio signal using standard ray propagation theory. Then we relate this theoretical basis to GPS occultation raw observations by untangling the occultation measurements of phase and the transmitting and receiving satellite trajectories. Thirdly we relate the observations to atmospheric param- 
eters of interest by inverting the data from bending analysis through refractivity profiles all the way to estimates of temperature, pressure, and water vapor profiles. Fourthly, we give theoretical estimates for the errors incurred at each stage of data processing so that these measurements can be fully utilized in numerical weather data assimilation and climate applications. Finally, we have included an overview of some advanced techniques of occultation data analysis that are currently under development.

The paper begins with an overview of the Global Positioning System (Section 2), followed by the basic geometric optics theory of radio occultations (Section 3) including resolution (Section 4). We then discuss issues related to atmospheric attenuation (Section 5) and multipath (Section 6) and how the resolution of the observations may be improved by reducing the effects of diffraction (Section 7). The meaning of refractivity and its conversion into density, temperature, pressure and water vapor are described in Section 8. We identify and discuss some of the important issues in processing real data in Section 9. We conclude with a summary of expected accuracy and the accuracy of initial results derived from the GPS/MET experiment in Sections 10 and 11 respectively.

\section{THE GLOBAL POSITIONING SYSTEM}

The Global Positioning System (GPS) consists of 24 satellites, distributed in six orbital planes about the globe. Each satellite orbit is circular, with an inclination of $\sim 55^{\circ}$, a period of 12 hours and an altitude of $20,200 \mathrm{~km}$. Although designed as a navigation aid by the U.S. Air Force, the number of high-precision civilian applications of GPS has grown steadily with time and now includes the study of the atmosphere. Each GPS satellite continuously transmits right-hand circularly polarized signals at two $L$ band frequencies, $f_{1}=1575.42 \mathrm{MHz}$ (L1) and $\mathrm{f}_{2}=1227.60 \mathrm{MHz}$ (L2), corresponding to wavelengths of $19.0 \mathrm{~cm}$ and $24.4 \mathrm{~cm}$, respectively. These signals are modulated at a frequency of $10.23 \mathrm{MHz}$ by a pseudo-random precision ranging code ( $\mathrm{P}$ code) (Spilker, 1978). The LI carrier signal is also modulated at a frequency of $1.023 \mathrm{MHz}$ by a coarse/acquisition (C/A) ranging code. The $\mathrm{L} 1$ and $\mathrm{L} 2$ signals received from each GPS satellite can be written as (Spilker, 1980):

$$
S_{L 1}(t)=\sqrt{2 C_{C / A}} D(t) X(t) \sin \left(2 \pi f_{1} t+\theta_{1}\right)+\sqrt{2 C_{P 1}} D(t) P(t) \cos \left(2 \pi f_{1} t+\theta_{1}\right)
$$

and

$$
S_{L 2}(t)=\sqrt{2 C_{P 2}} D(t) P(t) \cos \left(2 \pi f_{2} t+\theta_{2}\right)
$$

with

$C_{C / A}, C_{P 1}$ the received power of the in-phase and quadrature components of the L1 signal, respectively;

$C_{P 2}$ the received power of $\mathrm{L} 2$;

$D(t)$ an amplitude modulation for $\mathrm{L} 1$ and $\mathrm{L} 2$ containing navigation data;

$X(t)$ a pseudo-random sequence of \pm 1 --known as clear acquisition or C/A code-modulating the in-phase component of $\mathrm{L} 1$ at a rate of $1.023 \mathrm{Mhz}$;

$\mathrm{P}(\mathrm{t})$ a pseudo-random sequence of \pm 1 --known as $\mathrm{P}$-code-modulating the quadrature component of $\mathrm{L} 1$ and $\mathrm{L} 2$ at a rate of 10.23 Mhz. 
A properly equipped receiver will detect amplitude, pseudo-range ${ }^{3}$ and phase measurements for each of the C/A, L1 P-code (P1) and L2 P-code (P2) signals. The C/A and P1 measurements essentially contain identical information, however $\mathrm{C} / \mathrm{A}$ is preferred over $\mathrm{P} 1$ because its power is stronger by $3 \mathrm{~dB}$ and is not encrypted. Therefore, the basic observables used during an occultation experiment are the C/A phase and the P2 phase measurements between the low-Earth orbit (LEO) satellite and the occulting GPS satellite.

In contrast to upward-looking observations by ground-based GPS receivers, the spaceborne radio occultation observations are made in a limb-viewing geometry where the atmosphere lies between the transmitting source and the Low Earth Orbiting (LEO) receiver as shown schematically in Fig. 1. The variables shown in Fig. 1 are described in Section 3.5. From the standpoint of the receiver, an occultation occurs whenever a GPS satellite rises or sets such that the ray path traverses the Earth's atmospheric limb. With 24 GPS satellites, a single GPS receiver in a near-polar orbit at $800 \mathrm{~km}$ will observe over 500 occultations per day, distributed fairly uniformly about the globe (Fig. 2). All of these occultations provide useful atmospheric data. The horizontal distribution of coverage depends chiefly on the inclination and period of the LEO satellite orbit. Coverage is improved by placing additional receivers in orbit. The proposed set of 8 COSMIC GPS receivers spread in 8 low Earth orbits will provide approximately 4,000 daily occultations yielding an average of one profile every $360 \mathrm{~km}$ square per day.

The orbital motion of the two spacecraft rather than the orientation of the transmitting and receiving antennas provide the limb scanning motion during an occultation. Furthermore, the direction from which the signal arrived is determined from the knowledge of observational geometry and measured Doppler shift rather than knowledge of the antenna orientations. Together, these two facts enable acquisition of the occulted GPS signals with broad beam antennas, dramatically reducing the instrumentation and spacecraft requirements and design complexity and cost.

\section{ATMOSPHERIC BENDING AND REFRACTIVE INDEX PROFILE RETRIEVAL: THEORY}

\subsection{Refractivity}

We begin our discussion of retrieval theory with a brief overview of the index of refraction in the atmosphere. The index of refraction, $n$, in some medium is defined as the speed of light in a vacuum divided by the speed of light in the medium. In the atmosphere, the index of refraction is very close to unity such that it is usually discussed in terms of refractivity defined as $N=(n-1) \times 10^{6}$. At microwave wavelengths, the dependence of refractivity on atmospheric variables is expressed approximately as:

${ }^{3}$ Pseudorange is an absolute measurement of group delay between the time a signal is transmitted and received. It is the sum of the actual range between the transmitter and the receiver, atmospheric and ionospheric delays and transmitter and receiver clocks offsets. 


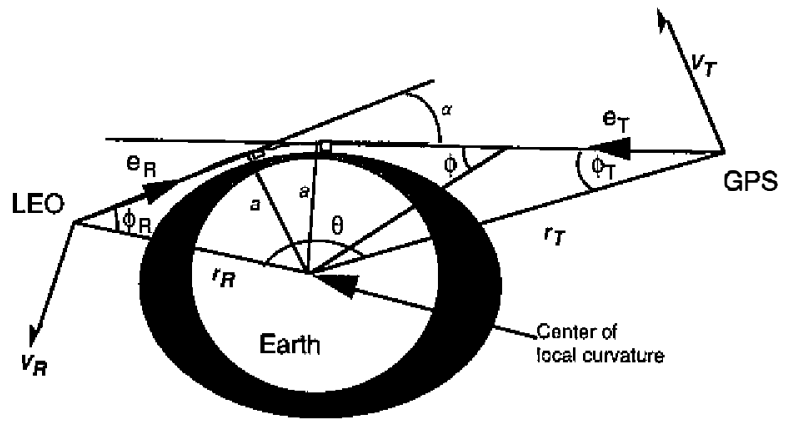

Fig. 1. Schematic representation of the geometry of a GPS atmospheric occultation. The variables are defined in Section 3.5.

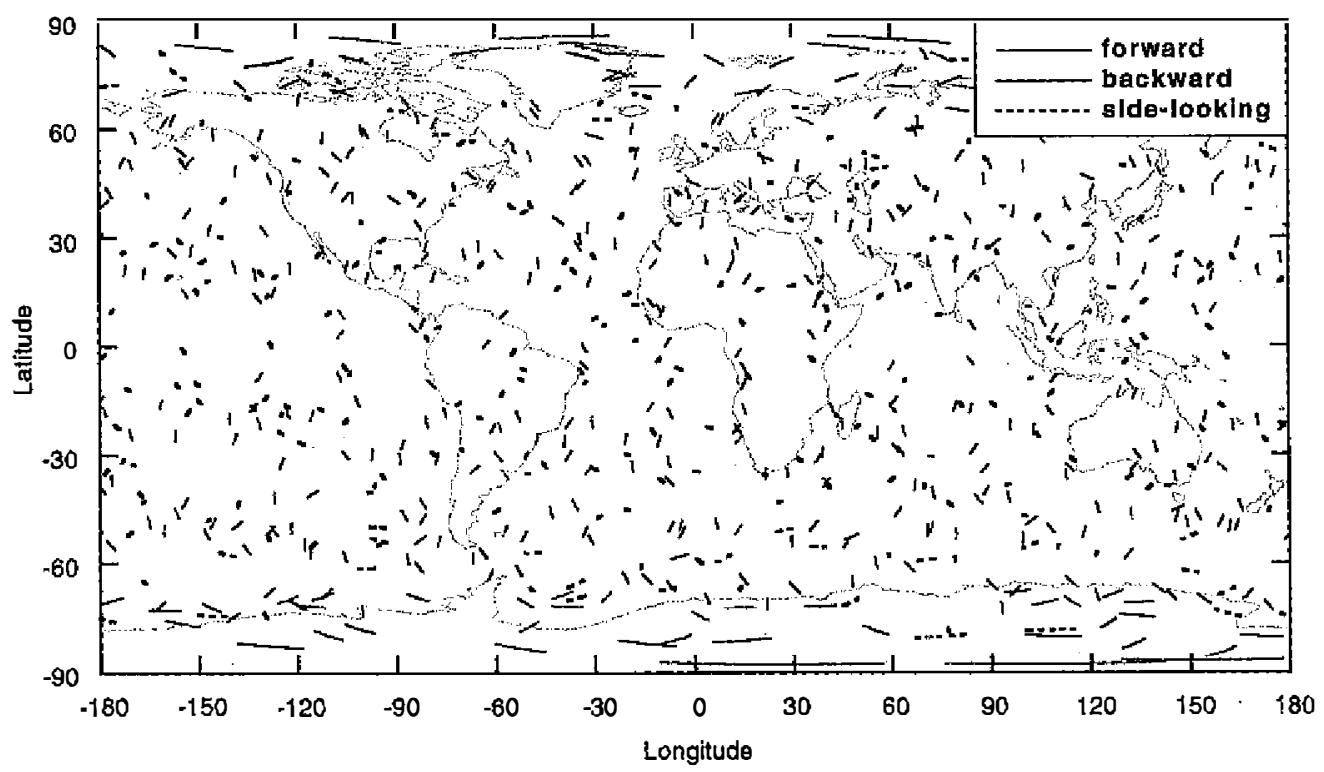

Fig. 2. Distribution of occultations observed in one day by a polar orbiter at 800 $\mathrm{km}$ altitude viewing 24 GPS satellites. Forward indicates rising occultations occuring within $\pm 45^{\circ}$ of the spacecraft velocity vector. Backward indicates setting occultations occurring within $\pm 45^{\circ}$ of the satellite antivelocity direction. Side-looking refers to all other occultations.

$$
N=77.6 \frac{P}{T}+3.73 \times 10^{5} \frac{P_{w}}{T^{2}}-4.03 \times 10^{7} \frac{n_{e}}{f^{2}},
$$

where $T$ is temperature in Kelvin, $P$ and $P_{w}$ are total pressure and partial pressure of water vapor in mbar, $n_{e}$ is free electron density in the ionosphere in electrons per cubic meter and $f$ is the signal frequency in $\mathrm{Hz}$. The GPS satellites continuously radiate signals at two frequencies, $f_{1}$ and $f_{2}$ discussed in the previous section, in order that users can separate the frequency dependent propagation delays due to ionospheric refractivity from other effects like the temperature and moisture of the neutral atmosphere. The sources of terms in (3) are discussed in Section 8. 


\subsection{Ray Optics}

The inversion of atmospheric occultation data is based largely on the concept of ray optics where light is described as propagating along lines called raypaths. Light waves propagate in a direction orthogonal to the geometrical wavefronts defined as the surfaces on which the signal phase is constant. Lines representing these trajectories are called raypaths. From this definition the differential equation of the raypaths can be derived (Born and Wolf, 1980) as

$$
\frac{d}{d s}\left(n \frac{d \boldsymbol{r}}{d s}\right)=\operatorname{grad} n,
$$

where $r$ is position along the raypath (bold indicates a vector), grad is the gradient and $d s$ is an incremental length along the raypath such that

$$
d r=s d s,
$$

where $s$ is the unit vector in the direction of the raypath. (4) is known as the Eikonal equation which is derivable from a least action (optical path) principle and works in the geometric optics approximation under the constraint that all features in the refracting medium are larger than the Fresnel size (see Section 4.3).

\subsection{Bouguer's Rule}

Consider the change in the quantity, $\boldsymbol{r} \times n \boldsymbol{s}$, along the raypath given as

$$
\frac{d}{d s}(r \times n s)=\frac{d r}{d s} \times n s+r \times \frac{d}{d s}(n s),
$$

From (5), the first term on the right is zero and from (4), (6) becomes

$$
\frac{d}{d s}(\boldsymbol{r} \times n s)=\boldsymbol{r} \times \operatorname{grad} n,
$$

(7) shows that only the non-radial portion of the gradient of index of refraction contributes to changes in $\boldsymbol{r} \times n s$. Therefore, the magnitude of $\boldsymbol{r} \times n s$ which is

$$
r n \sin \phi \equiv a,
$$

where $\phi$ is the angle between raypath and the radial direction, is constant if $n=n(r)$. The constancy of $a$ in (8) in a spherically symmetric atmosphere is known as Bouguer's rule and represents Snell's law in a spherically symmetric medium.

To evaluate the quality of Bouguer's law as an approximation in the Earth's atmosphere consider the following. The largest values of $n$ and hence the largest horizontal changes in $n$ occur near the surface where raypaths can traverse very dry to very wet conditions as might occur when the signal path crosses a coastline. In extreme conditions, the wet index of refraction might be $\sim 1.0004$ whereas in the dry region $n$ might be as low as $\sim 1.0003$ causing a horizontal change in $n$ along the raypath of $0.01 \%$. The resulting $0.01 \%$ change in $a$ of $\sim 0.6$ $\mathrm{km}$ is so small that Bouguer's rule is generally a good approximation for the Earth's atmosphere which simplifies interpretation of the occultation observations. 


\subsection{Bending Angle}

The change in the raypath direction accumulated along the raypath is defined as the bending angle. From (4) and (5), the rate of change in direction along the raypath is given as

$$
\frac{d s}{d s}=\frac{\operatorname{grad} n}{n}-s \frac{1}{n} \frac{d n}{d s}=\frac{\operatorname{grad}_{\perp s} n}{n},
$$

Therefore the bending is due to and in the direction of the portion of $\operatorname{grad} n$ which is orthogonal to the raypath direction, i.e., the projection of $\operatorname{grad} n$ in the plane perpendicular to $s$ $\left(\equiv \operatorname{grad}_{\perp S} n\right)$. We can define a local coordinate system where $x$ is orthogonal to $r$ and lies in the plane defined by $\boldsymbol{s}$ and $\boldsymbol{r}$ and $\boldsymbol{y}$ is in the direction orthogonal to the $\boldsymbol{r}$ - $\boldsymbol{x}$ plane. The bending angle change, $d \alpha$, is $|\mathbf{d s}| /|\mathbf{s}|$ such that the bending angle contribution along the raypath is

$$
d \alpha=\frac{d s}{n}\left[\left(\frac{\partial n}{\partial r} \sin \phi+\frac{\partial n}{\partial x} \cos \phi\right)^{2}+\left(\frac{\partial n}{\partial y}\right)^{2}\right]^{1 / 2}
$$

The largest gradients of refractivity are generally encountered in the deepest part of the atmosphere near the tangent point along the raypath where $\sin \phi$ is $\sim 1$. The $\cos \phi$ term will be very small near the tangent point such that the greatest horizontal gradient contribution will come from the gradient in the $y$ direction. Because the aspect ratio of dynamical features in the atmosphere is governed by the planetary rotation rate and Brunt frequency (i.e., static stability), magnitudes of horizontal gradients are generally much smaller than those of vertical gradients. An extreme case of horizontal bending can occur when a raypath travels along the edge of a several hundred kilometer, linear water vapor structure that has a large horizontal gradient along its edge. According to the ECMWF global analyses, such features exist in the boundary layer with horizontal gradients of $n$ roughly equal to $5 \times 10^{-7} \mathrm{~km}^{-1}$. The resulting horizontal bending angle contribution due to the horizontal gradient is roughly $0.005^{\circ}$, which is less than $1 \%$ of the near surface bending angle due to the vertical gradients. The horizontal displacement of such a raypath would be $\sim 0.4 \mathrm{~km}$. Therefore, as with Bouguer's rule, to a good first approximation the atmosphere is spherically symmetric.

The spherical symmetry approximation allows the spherically symmetric version of (10) to be written as

$$
d \alpha=d r\left(\frac{d \ln (n)}{d r} \frac{a}{\sqrt{n^{2} r^{2}-a^{2}}}\right),
$$

and the total refractive bending angle becomes

$$
\alpha(a)=2 \int_{r_{t}}^{\infty} \mathrm{d} \alpha=2 a \int_{r_{i}}^{\infty} \frac{1}{\sqrt{n^{2} r^{2}-a^{2}}} \frac{\mathrm{d} \ln (\mathrm{n})}{\mathrm{dr}} \mathrm{dr},
$$

where $r$ is distance from the center of curvature and the integral is over the portion of the atmosphere above $r_{t}$, the radius at the tangent point of the raypath.

The forward calculation of $\alpha(a)$ given $n(r),(12)$, can be inverted by using an Abelian 
transformation to express $n(r)$ in terms of $\alpha$ and $a$ (Fjeldbo et al., 1971).

$$
\mathrm{n}(r)=\operatorname{Exp}\left[\frac{1}{\pi} \int_{\mathrm{a}_{1}} \frac{\alpha}{\sqrt{a^{2}-a_{1}^{2}}} \mathrm{~d} a\right],
$$

where $a_{1}=n r$ is the impact parameter for the ray whose tangent radius is $r$. Given $\alpha(a),(13)$ can be evaluated numerically. Note that $r$ is derived as

$$
r=\frac{a_{1}}{n(r)} .
$$

As discussed, the ray paths for a given occultation do not generally scan the atmosphere vertically and are not quite coplanar such that measurements of $\alpha(a)$ will be affected by tangential refractivity gradients and occultation geometry, and (13) will introduce systematic errors into retrieved profiles of $n(r)$. While Kursinski et al. (1997) show that the Abel transform will generally be quite accurate as an initial retrieval scheme for GPS occultations of the Earth's atmosphere, improved accuracy can be accomplished by incorporating knowledge of local horizontal structure into the profile retrieval process. Such a scheme has been developed for the special case of elliptical symmetry to invert radio occultation observations of the oblate atmospheric structure of the outer planets (Lindal, 1992). More general approaches are evolving to take advantage of multiple occultations and a priori information (Gorbunov and Sokolovskiy, 1993; Eyre, 1994; Hajj et al., 1994; Zou et al., 1999; Ahmad and Tyler, 1999).

\subsection{Derivation of $\alpha(\mathrm{A})$ from Doppler Shift Measurements}

The effect of atmospheric bending on a GPS signal received at a LEO receiver can be measured as an additional Doppler shift relative to that expected for a straight line signal path. Subject to the assumption of local spherical symmetry, the Doppler shift can be combined with satellite position and velocity vector knowledge to derive $\alpha(a)$. Using the geometry and notation of Fig. 1, the Doppler shift $f_{d}$ in the transmitter frequency $f_{T}$, measured at the receiver produced by the projection of the spacecraft velocities onto the ray paths, is given by

$$
\begin{aligned}
f_{d} & =\frac{f_{T}}{c}\left(\mathbf{V}_{\mathbf{T}} \bullet \hat{\mathbf{e}}_{\mathbf{T}}+\mathbf{V}_{\mathrm{R}} \bullet \hat{\mathbf{e}}_{\mathrm{R}}\right) \\
& =-\frac{f_{T}}{c}\left(\mathrm{~V}_{\mathrm{T}}^{\mathrm{r}} \cos \phi_{T}+\mathrm{V}_{\mathrm{T}}^{\theta} \sin \phi_{\mathrm{T}}+\mathrm{V}_{\mathrm{R}}^{\mathrm{r}} \cos \phi_{\mathrm{R}}-\mathrm{V}_{\mathrm{R}}^{\theta} \sin \phi_{\mathrm{R}}\right),
\end{aligned}
$$

where $\mathbf{V}_{\mathbf{R}}$ and $\mathbf{V}_{T}$ are receiver and transmitter velocity vectors, $\hat{\mathbf{e}}_{\mathbf{R}}$ and $\hat{\mathbf{e}}_{\mathbf{T}}$ are unit vectors representing the direction of the ray path at the receiver and wansmitter, and $c$ is the velocity of light. The RHS of (15) has resolved the velocity vectors into radial (superscript $r$ ) and tangential (superscript $\theta$ ) components, and $\phi_{T}$ and $\phi_{R}$ are the angles between the ray path and the spacecraft position vector at the transmitter and receiver (Fig. 1). In writing (15), the relativistic contributions to the received frequency have been eliminated on the basis of knowledge of orbital geometry and Earth's gravity field (see Section 9).

As $r \rightarrow$ ninfinity, $n \rightarrow$ unity, so that the constant term in (8) must equal $a$, the impact 
parameter, defined in Fig. 1. Furthermore, at the ray tangent radius $r_{i}, \sin \phi=$ unity so that $a=$ $n r_{i}$. From Bouguer's rule and the geometry of Fig. 1, we have:

$$
\begin{gathered}
r_{T} \sin \phi_{\mathrm{T}}=r_{R} \sin \phi_{\mathrm{R}}=a, \\
\alpha=\phi_{r}+\phi_{R}+\theta-\pi,
\end{gathered}
$$

where $r_{R}$ and $r_{T}$ are the distances of the receiver and transmitter from the center of curvature, and $\theta$ is the angle between the transmitter and the receiver position vectors. Given the precise transmitter and receiver position and velocity vectors, transmitter signal frequency, and the occultation Doppler shift, $\alpha(a)$ can be derived iteratively from (15), (16), and (17) by eliminating $\phi_{T}$ and $\phi_{R}$. Note that because Bouguer's rule is invoked, this derivation is only exact under the assumption of local spherical symmetry. Errors in $\alpha$ and $a$ due to this assumption have a small effect on retrieved refractive index profiles compared with the use of the Abel transform inversion.

\subsection{Center of Curvature}

Interpretation of the Doppler shift observed during an occultation requires knowledge of the observation geometry. Bending is defined largely by the geometry of the refractivity contours. Because of gravity and hydrostatic balance, refractivity gradients are oriented to first order along the direction of gravity and contours of constant refractivity therefore follow approximately the shape of the geoid. Because the Earth is spinning, its shape is oblate which causes the local curvature center of refractivity contours around the globe to vary with respect to the center of the Earth. The effect of oblateness on radio occultation observations was realized soon after the first such observations of Jupiter's atmosphere were interpreted ignoring oblateness (Kliore et al., 1974, 1975) and yielded temperatures in the deep troposphere several hundred Kelvin hotter than other observations and theory (Eshleman, 1975). Subsequently the average center of curvature as defined by the planet's shape in the vicinity of the tangent point of the occulted ray paths brought the results in line with other observations and theory (Kliore et al., 1976).

Because Earth's oblateness is relatively small, with an equatorial radius roughly $20 \mathrm{~km}$ larger than its polar radius, the same approach is used by adjusting the coordinate center to be the center of the circle tangent to the geoid at the closest approach point of a given ray path rather than the center of the Earth. Since the shape of the geoid varies by less than $100 \mathrm{~m}$ with respect to an ellipsoid, an ellipsoid can be used to represent the geoid shape. To find the approximate center of curvature, we first find the approximate occultation plane as defined by the transmitter, receiver and normal to the ellipsoid in the vicinity of the ray path tangent point. The circle is then found which fits the contour of the ellipsoid in the occultation plane at the ray path tangent point. The center of this circle then defines the center of curvature and coordinate center used to interpret the occultation Doppler data and derive the profile of $\alpha$ and $a$. The adjustment in coordinate center away from the center of the Earth reduces errors in $a$ caused by the non-spherical geoid from $\sim 40 \mathrm{~km}$ to $\sim 0.1 \mathrm{~km}$. 


\subsection{Derivation of $\alpha(A)$ from Amplitude Measurements}

For completeness, we note that the change in signal intensity observed during an occultation can also be used to derive atmospheric refractivity structure. The attenuation mechanisms affecting propagation of occulted signals across the atmospheric limb are absorption by atmospheric constituents, extinction by particle scattering and defocusing. As such, planetary occultations of stars have long been used to characterize the upper regions of planetary atmospheres. As discussed by Kursinski et al. (1997), atmospheric absorption at L-band wavelengths is dominated by the far wings of pressure-broadened molecular oxygen and water vapor lines, with the contribution of oxygen being at least an order of magnitude greater than that of water vapor. For a limb path, net L-band optical depth varies roughly as $P^{2} / T$ where $P$ and $T$ are the pressure and temperature at the tangent height. Calculations using absorption spectra from Chahine et al. (1983) show that absorption by molecular oxygen reduces GPS signal intensities by a factor of 2 , for a limb path with a tangent height at the surface. Extinction caused by realistic suspensions of water droplets or ice particles in the atmosphere is negligible at L band wavelengths (Kursinski, 1997; Liebe, 1989).

In a geometric optics framework, defocusing is due to the change in direction between adjacent raypaths caused by the atmosphere. Adjacent raypaths passing through a region where bending increases at lower altitudes spread apart vertically causing the signal energy flux to be spread over a larger area causing the received signal intensity to decrease. The vertical defocusing effect is expressed as

$$
\left[1-\frac{d \alpha}{d a} \frac{L_{r} L_{t}}{L_{r}+L_{t}}\right]^{-1} \equiv M
$$

where $L_{l}$ and $L_{r}$ are the distances from the limb to the transmitter and receiver respectively. (We will use the definition of $M$ in later sections.) There is also slight focusing in the direction orthogonal to the occultation plane due to the curvature of the Earth that can be expressed as

$$
\left[1-\frac{\alpha}{R_{E}} \frac{L_{r} L_{i}}{L_{r}+L_{t}}\right]^{-1}
$$

where $R_{E}$ is the radius of Earth. Combining the absorption and defocusing terms yields

$$
\frac{I}{I_{0}}=\left[1-\frac{d \alpha}{d a} \frac{L_{r} L_{t}}{L_{r}+L_{t}}\right]^{-1}\left[1-\frac{\alpha}{R_{E}} \frac{L_{r} L_{t}}{L_{r}+L_{t}}\right]^{-1} \exp \left[-b \frac{p^{2}}{T}\right],
$$

where $I$ is the observed intensity, $I_{0}$ is the intensity which would have been observed in the absence of the occulting atmosphere, $P$ and $T$ are the pressure and temperature at the raypath tangent height in mb and kelvin respectively and $b=2 \times 10^{-4} \mathrm{~K} \mathrm{mb}^{-2}$. The defocusing effect due to $d \alpha / d a$ is generally much greater than the slight $\alpha / R_{E}$ focusing effect because the bending angle scale height is much smaller than the radius of the Earth.

From (18) and (19) the bending angle versus asymptotic miss distance can be derived from a profile of occulted signal amplitudes as follows. Initially one assumes a pressure - 
temperature profile. The following steps are to

1. isolate and derive $d \alpha / d a$ from $I / I$, via (19)

2. combine $d \alpha / d a$ with (16) and (17) to derive an $\alpha(a)$ profile

3. derive a refractvity profile via (13)

4. derive a new pressure - temperature profile (see Section 8 )

One then repeats steps 1 through 4 using the new pressure - temperature profile derived in step 4 until convergence is achieved.

Doppler measurements are preferable to amplitude measurements for deriving atmospheric profiles because they are more sensitive in general. Signal intensity cannot be measured as accurately as frequency. Doppler measurements provide a direct measure of $\alpha(a)$ whereas amplitude provides the derivative that must be integrated to obtain $\alpha(a)$. In the case of a stellar occultation where the signal source is a broad band, incoherent source without a welldefined phase, amplitude measurements must be used to infer refractivity information. Such intensity-only measurements have a further weakness because they have no ability to discriminate between atmospheric multipath, where the received signal is the superposition of several raypaths which exist simultaneously between the transmitter and receiver (see Section 6).

\section{RESOLUTION OF OCCULTATION OBSERVATIONS}

Here we discuss the limits to the spatial resolution of atmospheric profiles retrieved from GPS radio occultation measurements largely following the discussion of Kursinski et al. (1997). The vertical resolution associated with a single, instantaneous bending measurement is determined by the contribution of individual atmospheric layers to net bending along the ray path (12). Better vertical resolution can be retrieved from a profile of closely separated measurements, subject to limits imposed by diffraction.

\section{1 Vertical Resolution - Individual Measurements of Bending Angle}

The vertical variation of the contribution of atmospheric layers to an individual bending angle measurement (one in a sequence of measurements taken during an occultation) based on the atmospheric profile shown in Fig. 3 is illustrated in Fig. 4 as a function of ray path tangent altitude (from Kursinski et al., 1997). The curves on the right show the relative contribution of each altitude interval to net atmospheric bending for several tangent altitudes. These contribution functions are strongly peaked at the tangent altitude due to the limb sounding geometry and exponential variation of refractivity with altitude ((11) and (3)). Vertical resolution, defined here as the vertical interval above the tangent height contributing $50 \%$ to net bending, is plotted as a function of tangent altitude in the left-hand side of Fig. 4. For the atmospheric profile used in Fig. 4, the $50 \%$ bending interval varies typically from 1.2 to $2.0 \mathrm{~km}$. The smallest $50 \%$ interval of $\sim 0.6 \mathrm{~km}$ occurs when the ray path tangent height coincides with the tradewind inversion near $3.8 \mathrm{~km}$ in Fig. 3. At this altitude, the vertical refractivity gradient is very large because of the extreme change in humidity across the top of the inversion that concentrates the bending and improves the vertical resolution in a region where it is needed 
most.

\section{2 Horizontal Resolution - Individual Measurements of Bending Angle}

Horizontal resolution associated with a single bending measurement is defined by writing the bending contribution in terms of distance along the raypath using the spherically symmet-

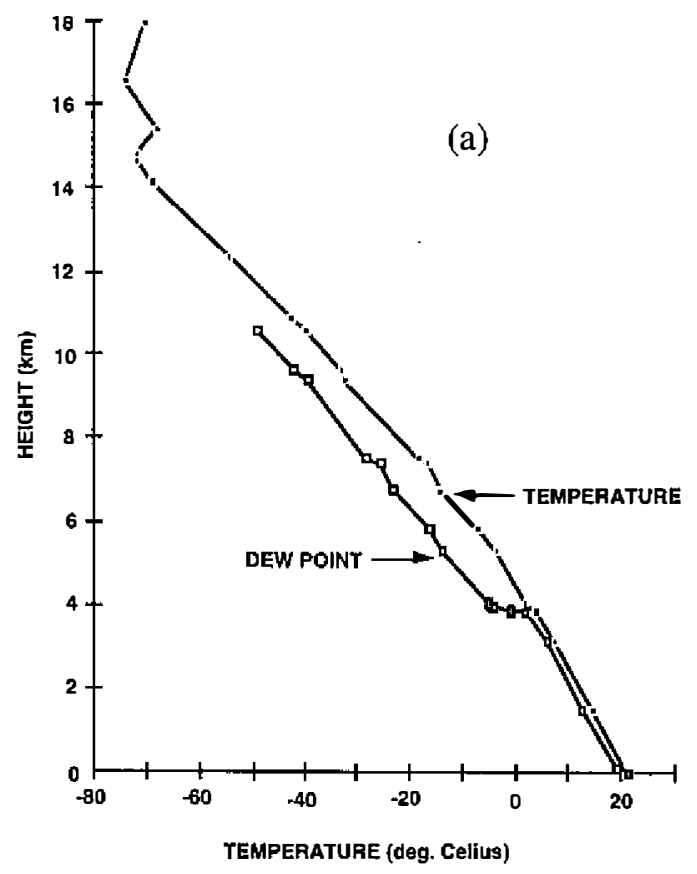

Fig. 3. Radiosonde profile ascent 12:00 UT, 11 July 1991, Hilo, Hawaii. (a). Temperature and dew point versus height. (b). Profiles of the dry, moist and total refractivity derived from the radiosonde profile via (46).

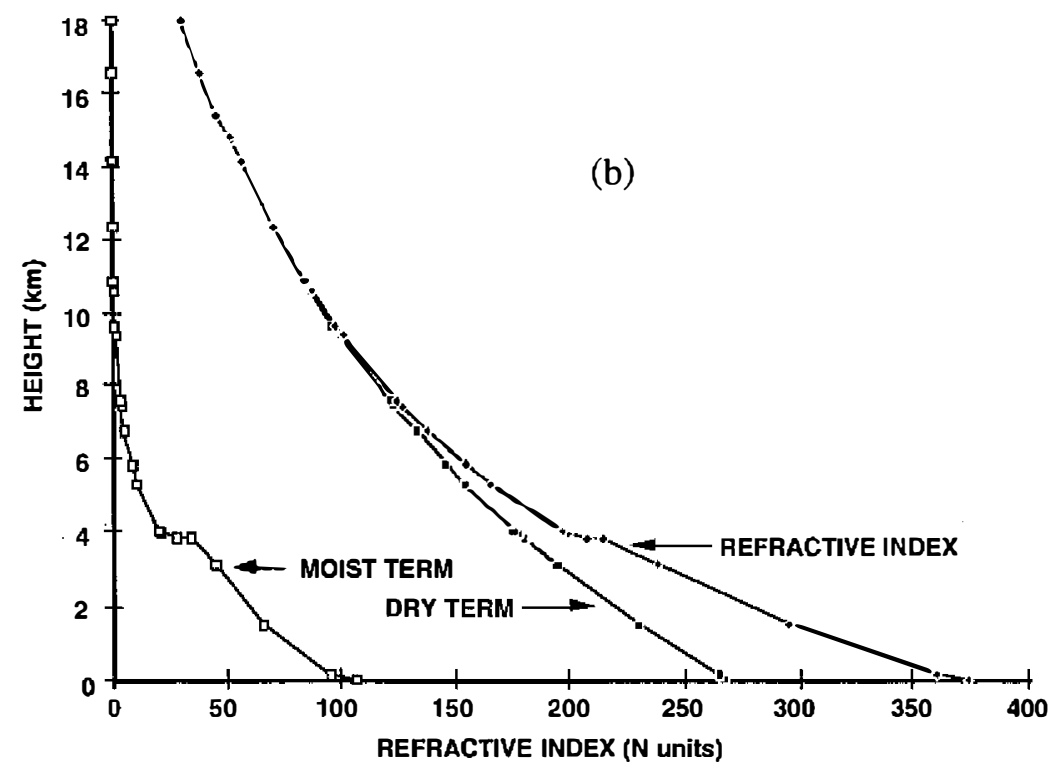


ric form of (11)

$$
d \alpha=\frac{d s}{n} \frac{d n}{d r} \sin \phi .
$$

Assuming refractivity varies exponentially with height, the change in bending along the path is given approximately as

$$
\frac{d \alpha}{d s} \cong-\frac{N_{t}}{H n} \exp \left(-\frac{s^{2}}{2 r_{t} H}\right)\left(1-\frac{s^{2}}{2 r_{t}^{2}}\right),
$$

where $s$ is distance along the ray path measured from the ray path tangent point, $r$, and $N_{t}$ are the radius and refractivity respectively at the tangent point and $H$ is the refractivity scale height. (20) shows the bending contribution along the ray path is approximately Gaussian. Given typical refractivity scale heights of 6 to $8 \mathrm{~km}$, half the bending is contributed within $\pm 200 \mathrm{~km}$ of the raypath tangent point.

\subsection{Vertical Resolution - Diffraction Limit}

The vertical resolution of refractivity profiles retrieved from profiles of radio occultation bending angle measurements can be significantly better than that of the individual bending angle measurements given that the bending angle measurements in a profile are closely spaced

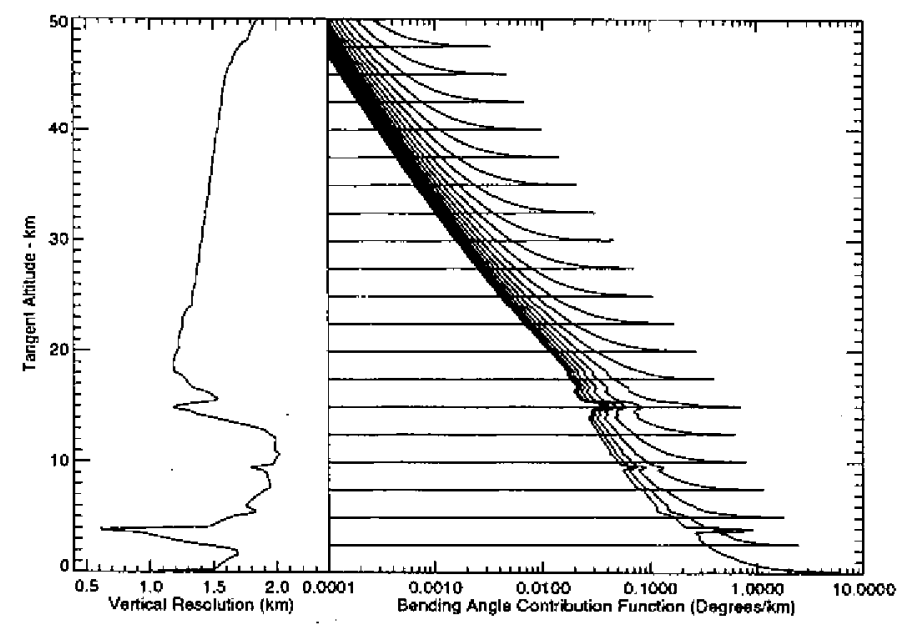

Fig. 4. Bending contribution functions (solid lines) and vertical resolution profile (dotted line) for individual radio occultation bending angle measurements plotted as a function of altitude. Resolution is defined here as the vertical interval between the ray path tangent height and the height at which half of the total bending has been accumulated. These curves are calculated for the refractivity profile in Fig. 3, assuming spherical symmetry. 
and measured precisely, and can be inverted with a scheme that correctly represents the contribution of different levels to bending angle. All of these conditions are met for GPS occultations measured by a LEO satellite provided the atmosphere is locally spherical in structure such that the Abel transform inversion of (13) applies. Under these conditions, the ultimate limits to vertical and horizontal resolution of retrieved profiles are set by diffraction.

The geometric optics representation of signal propagation and the Abel inversion theory discussed in Section 3 assume that signals follow dimensionless ray paths between the transmitter and receiver. A ray path is the path of minimum phase delay relative to the delay associated with neighboring, physically realizable paths. However, the geometric optics and ray path framework is an approximation to Fresnel diffraction in the limit of zero wavelength. Because signals originate at a point source and are detected by a receiver phase-locked to the minimum phase delay, the cross-beam sampling at the limb takes the form of concentric Fresnel zones of positive and negative response centered on the ray path. Since the contributions of adjacent Fresnel zones cancel, the effective sampling can be represented approximately by the first Fresnel zone (Born and Wolf, 1980). Hinson and Magalhaes (1991) showed that in the thin phase screen approximation the ray optics approximation is valid for retrieving vertical atmospheric structures whose wavelength is the diameter of the first Fresnel zone or larger.

The first Fresnel zone is centered on the path of minimum phase delay and is defined as the region within which the path lengths are within a half wavelength of the path of minimum phase delay. In the absence of significant atmospheric bending, geometry dictates that the diameter of the first Fresnel zone, $F_{0}$, at the ray path tangent level is given by:

$$
\left.F_{0}=2\left[\lambda L_{T} L_{R} /\left(L_{T}+L_{R}\right)\right]^{1 / 2} \mathrm{H} 2\left[\lambda L_{R}\right]^{1 / 2} \text { (if } L_{T} \gg L_{R}\right),
$$

where $\lambda$ is the GPS signal wavelength, and $L_{T}$ and $L_{R}$ are the distance from the transmitter and receiver to the limb respectively. $L_{r}$ is approximately $25,800 \mathrm{~km}$ for the GPS transmitters and, for a receiver in a $700 \mathrm{~km}$ altitude orbit, $L_{R}$ is about $3,100 \mathrm{~km}$. At the GPS L1 wavelength of $19 \mathrm{~cm},(21)$ yields a value for $F_{0}$ of $1.4 \mathrm{~km}$.

The Fresnel zone decreases as refractivity increases such that diffraction-limited resolution improves deeper in the atmosphere in general. The size of the first Fresnel zone in the presence of bending is given approximately (Haugstad, 1978) as

$$
F=F_{0} \sqrt{M} \text {, }
$$

where $M$ is defined in (18). Figure 5 (from Kursinski et al., 1993, 1997) shows a representative profile of $M$ and vertical resolution calculated from a radiosonde profile from Hilo, Hawaii.

\subsection{Diffraction Limited Horizontal Resolution}

For the limb sounding geometry appropriate to GPS radio occultation, horizontal resolution may be defined by the distance traversed by the radio path as it enters and exits a layer having a vertical resolution of $\Delta Z$; in this case the horizontal and vertical resolution are related by the approximate expression: 


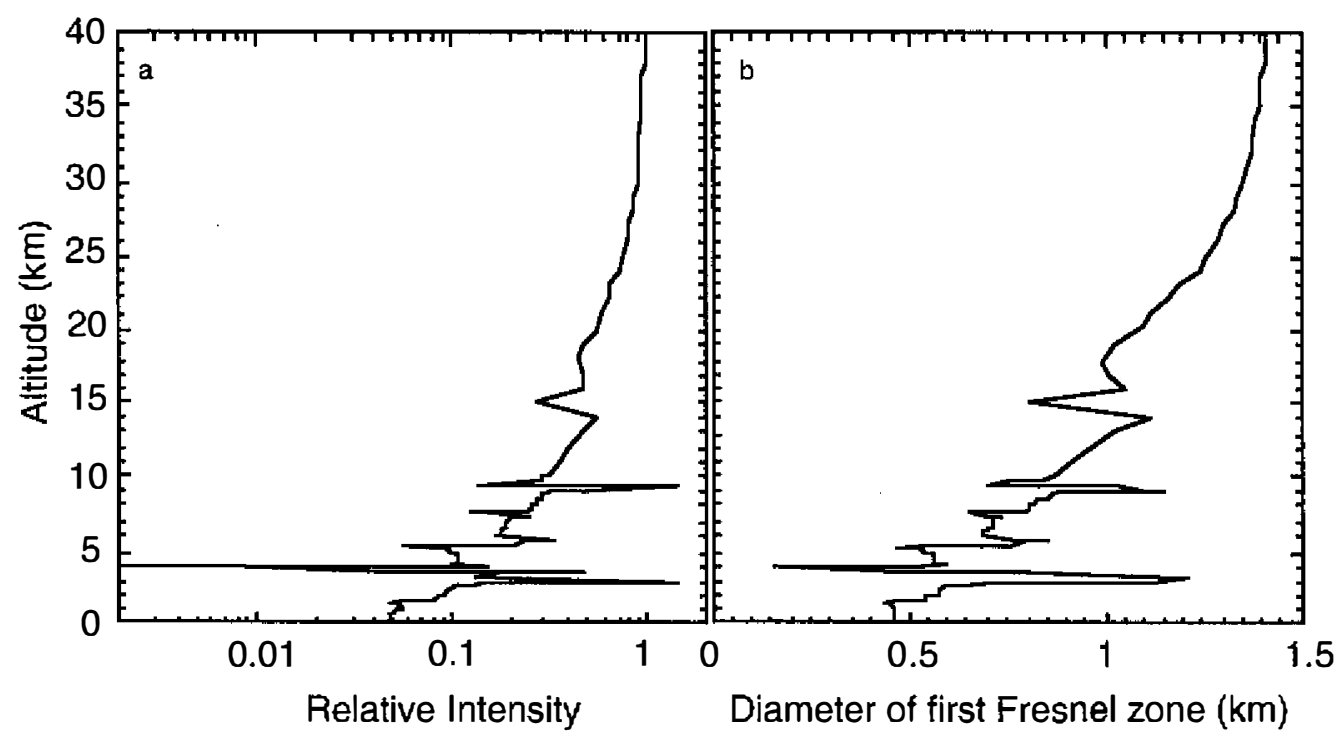

Fig. 5. Effect of atmospheric refractivity on signal intensity and diffraction limited resolution. (a). Variation of defocusing factor, $M$, with altitude (18). $M$ is calculated for the refractivity profile in Fig. $3 \mathrm{~b}$ assuming spherical symmetry. (b). The diameter of the first Fresnel zone defined by (20) and (21) plotted as a function of ray path tangent height for the refractivity profile in Fig. $3 \mathrm{~b}$.

$$
\Delta L \sim 2(2 R \Delta Z)^{1 / 2},
$$

where $\Delta L$ is horizontal resolution, $\Delta Z$ is vertical resolution, and $R$ is the radius of the atmosphere at the ray path tangent height. From (23) the horizontal resolution corresponding to a vertical resolution of $1.4 \mathrm{~km}$ is about $270 \mathrm{~km}$.

\subsection{Comparison between Fresnel and Aperture Diffraction}

The Fresnel diffraction limit differs dramatically from aperture diffraction that defines the diffraction resolution limit of limb-viewing passive radiance observations. The angular and vertical resolution of aperture diffraction are $\lambda / D$ and $L_{R} \lambda / D$ respectively where $\lambda$ is the signal wavelength and $D$ is the diameter of the receiving aperture. Setting aperture and Fresnel diffraction limits equal requires that $D$ equal $F_{0} / 4$, half the radius of the first Fresnel zone. The required aperture would be one half the radius of the first Fresnel zone, roughly $350 \mathrm{~m}$ using GPS wavelengths. The arc-minute half power beamwidth of such an aperture would require extremely accurate pointing and a very sophisticated spacecraft and attitude control system. While shorter wavelengths can be and are used to achieve resolution approaching that of GPS with smaller apertures, the large atmospheric opacities at shorter wavelengths limit penetration of such signals into the limb. Therefore the only practical approach to routinely limb sounding Earth's troposphere requires long wavelengths like GPS. 


\section{ATMOSPHERIC ATTENUATION AND CRITICAL REFRACTION}

In the lower troposphere, increasing pressure and humidity reduce the strength of the occulted signals while increasing their variability, a combination that could limit the altitude range of the occultation coverage. While these problems have, in fact, limited initial GPSMET coverage in the lower troposphere, they are surmountable with improvements in instrumentation and inversion techniques within the resolution constraints defined in Section 4. In order to acquire an occulted signal, the signal-to-noise intensity ratio (SNR) at the receiver must exceed a critical value of about 10. If SNR falls below this value, phase lock and the signal are lost. GPS transmitter power, receiver noise performance, observational geometry, and signal attenuation due to the atmosphere determine SNR. In the lower troposphere, signal acquisition can be further complicated by atmospheric multipath where several weak occulted signals exist simultaneously. The effects of atmospheric multipath and atmospheric attenuation and the SNR performance expected from a GPS receiver in low Earth orbit are described below.

\subsection{Atmospheric Attenuation due to Defocusing}

Atmospheric attenuation has been discussed briefly in Section 3. The dominant attenuation mechanism is defocusing which occurs when rapidly changing vertical refractivity gradients cause adjacent, nearly parallel rays entering the atmosphere to bend differentially and diverge, reducing (or sometimes enhancing) signal intensity at the receiver. The intensity of an occulted signal after defocusing is represented by (18). The variation of the defocusing factor $M$ with limb ray path tangent altitude, calculated for a vertical refractivity profile corresponding to a radiosonde ascent from Hilo, Hawaii, in July 1991, assuming spherical symmetry, is illustrated in Fig. 5a. Three general features of the curve should be noted. Firstly, a gradual, overall reduction in $M$ with decreasing altitude is caused by the exponential increase of bending angle with decreasing altitude. Secondly, rapid vertical variations in $M$ are associated with sharp vertical changes in the refractivity gradient caused primarily by vertical water vapor mixing ratio fluctuations. These variations provide a sensitive measure of the local refractivity scale height and humidity fluctuations. Finally, signals with ray path tangent heights that fall within certain vertical intervals are attenuated completely. In Fig. 5a, attenuation is complete at the top of the marine boundary layer near $3.8 \mathrm{~km}$ associated with the transition between the boundary layer and the overlying free troposphere.

\subsection{Critical Refraction Layers}

Complete attenuation occurs at ray path tangent altitudes where the vertical refractivity gradient becomes so large that the radius of curvature of the ray is smaller than the radius of curvature of the atmosphere, causing the ray to curve down into the surface. These conditions occur when the refractivity gradient $d N / d r<-10^{6} / R_{c}$, where $R_{c}$ is the radius of curvature of the atmosphere; for a mean value of $R_{c}, d N i d r \sim-0.16 \mathrm{~N}$-units $\mathrm{m}^{-1}$. The vertical atmospheric gradients required to satisfy this inequality can be examined by differentiating the dry and moist refractivity terms of (3) to give: 


$$
\frac{d N}{d r}=-\frac{b_{1} P}{H_{P} T}-\left(\frac{b_{1} P}{T^{2}}+\frac{2 b_{2} P_{W}}{T^{3}}\right) \frac{d T}{d r}+\frac{b_{2}}{T^{2}} \frac{d P_{W}}{d r},
$$

where $H_{P}$ is the pressure scale height. The three terms on the RHS of (24) represent the contributions of the vertical pressure, temperature, and water vapor mixing ratio gradients to $d N / d r$. For $T=280 \mathrm{~K}, P=850 \mathrm{mbar}$ and $P_{w}=9$ mbar (90\% relative humidity), conditions representative of the top of the marine boundary layer, the first term in (24) is $-0.03 \mathrm{~m}^{-1}$, demonstrating that realistic pressure gradients alone are incapable of generating critical refractivity gradients. The other terms, considered separately, require temperature gradients $\geq$ $+140 \mathrm{~K} / \mathrm{km}$ or water vapor partial pressure gradients $\leq-34 \mathrm{mbar} / \mathrm{km}$ to produce critical gradients. Of the vertical gradients in pressure, temperature, and water vapor contributing to $d N / d r$ via (24), only the water vapor gradient is capable of producing critical gradients. During the 1969 Atlantic Trade wind Experiment (ATEX), the transition depth at the boundary layer top was $\sim 200$ meters thick on average with mean changes in temperature and water vapor partial pressure across the region of $5 \mathrm{~K}$ and 6 mbar (Augstein et al., 1974). The resulting contributions to $d N / d r$ by the average temperature and moisture gradients are about $-0.03 \mathrm{~N}$-units $\mathrm{m}^{-1}$ and $-0.12 \mathrm{~N}$-units $\mathrm{m}^{-1}$, respectively. While the temperature contribution is relatively small, the mean water vapor gradient is $75 \%$ of the critical value, indicating a significant fraction of occultations will experience critical refraction in regions where a sharp inversion caps the marine boundary layer.

\subsection{Regions Where Critical Gradients Occur}

The maximum altitude of critical gradients can be constrained using a hypothetical layer with completely dry air above and $100 \%$ saturated air below and a vertical thickness of $\sim 100$ $\mathrm{m}$. Notice that the layer must extend $\sim 200 \mathrm{~km}$ or more horizontally to produce the critical bending. The saturation vapor pressure yielding the critical refraction gradient of $-34 \mathrm{mb} / \mathrm{km}$ is $3.4 \mathrm{mb}$, which corresponds to a temperature of $-5^{\circ} \mathrm{C}$. The annual mean climatological $-5^{\circ} \mathrm{C}$ temperature contour which envelops the region of possible critical refraction has a maximum altitude near $5 \mathrm{~km}$ at low latitudes and intercepts the surface near $60^{\circ}$ latitude (Peixoto and Oort, 1992).

One can argue that still lower saturation vapor pressures will produce critical gradients by simply narrowing the vertical extent of the layer. Decreasing the layer thickness to $10 \mathrm{~m}$ would raise the altitude at which critical refraction could occur by about $5 \mathrm{~km}$. However, since most of the received signal is contributed by the region within the first Fresnel zone, the vertical extent of the critical refraction layer must span a significant fraction of the diameter of the first Fresnel zone to produce extinction. Otherwise, the layer will only remove a portion of the signal flux causing scintillations plus some reduction in intensity but not extinction. The relatively large Fresnel zone resulting from a vantage point in low Earth orbit reduces the occurrence of extinction by smoothing over small scale radio holes which would plague observations made closer to the holes and/or at shorter wavelengths. Note that the extreme gradients at the top of the tradewind inversion decrease the Fresnel zone diameter to $\sim 150 \mathrm{~m}$ (Fig. $5 \mathrm{~b})$. Therefore, $100 \mathrm{~m}$ is a representative minimum interval thickness capable of producing 
extinction in spaceborne GPS observations and the $-5^{\circ} \mathrm{C}$ temperature contour defines the upper altitude limit of critical refraction for these observations.

This conclusion is consistent with the initial GPS/MET results. Despite a low antenna gain and receiver minimally modified from a ground-based geodetic receiver, the GPS/MET receiver demonstrated an ability to track routinely down to 1 to $3 \mathrm{~km}$ altitude. Initial GPS/ MET signal tracking at higher latitudes systematically extends to lower altitudes, and loss of lock at systematically higher altitudes at low latitudes in the initial GPS/MET occultations may coincide with the critical refraction estimate.but more probably reflects the low Signal to Noise Ratio (SNR) of the prototype receiver. The COSMIC GPS receivers with higher antenna gain and better signal tracking will improve substantially upon the GPS/MET limitations.

\subsection{Vertical Extent of Intervals of Critical Refraction}

As discussed by Kursinski et al. (1997), the vertical extent of the critical refraction interval can be derived from Bouguer's rule. In order for a ray to leave the atmosphere, $\phi$ in (8) must be less than or equal to $\pi / 2$ away from the tangent point, such that $n r>n_{r} r$, where the subscript " $t$ " refers to the ray path tangent where $\phi$ is $\pi / 2$. It follows that $r_{1} n_{1}=r_{0} n_{0}$ where the indices 1 and 0 refer to the tangent points of the exweme ray paths, respectively, at the top and bottom of the interval of complete attenuation. The vertical extent of the interval is given by

$$
\Delta r=r_{1}-r_{0}=r_{1}\left(1-n_{1} / n_{0}\right)=r_{1} \Delta n / n_{0} \sim r_{1} \Delta n,
$$

where $\Delta n=n_{0}-n_{1}$. The large change in index of refraction $\Delta n$ occurs primarily across the boundary layer transition region that has a vertical extent $\Delta z$. Therefore

$$
\Delta r \sim r_{1}(d n / d z)_{\mathrm{tr}} \Delta z=r_{1} \eta / R_{c} \Delta z \sim \eta \Delta z,
$$

where $\eta$ is the ratio between the large index of refraction gradient across the transition region $(d n / d z)_{t r}$ and the critical index of refraction gradient $1 / R_{C}$. The vertical interval of critical refraction therefore depends on both the magnitude and the vertical extent of the large refractivity gradient. As an example, an extremely large vertical moisture gradient observed by a radiosonde over Hilo, Hawaii (October 1, 19930000 UT) exceeded the critical gradient by a factor of 3 over a vertical interval $\Delta z \sim 80 \mathrm{~m}$ such that an occultation of this region would have observed a vertical interval of critical refraction $-240 \mathrm{~m}$ in extent.

\subsection{Derivation of Refractivity within and below Layers of Critical Refraction}

The existence of a critical refractivity interval raises the question of recovery of refractivity at altitudes within and below the interval. As the tangent height of a ray descends toward an interval where critical refraction occurs, bending increases and at the point of critical refraction, the bending angle becomes infinite. Analogously, the bending angle of a ray path whose tangent height lies at the bottom edge of the critical refraction interval is also infinite and bending immediately below the interval is finite. Since $r_{1} n_{1}=r_{0} n_{0}$, and $a_{1}=a_{0}$, the interval 
of critical refraction collapses to a singularity in the $\alpha$ versus $a$ profile. Accurate recovery of refractivity structure below the interval of critical refraction via the Abel transform requires first that the existence of the singularity be identified in the data using the characteristic disappearance of the signal and second that the numerical implementation of the Abel transform defined in (13) be capable of integrating accurately across the singularity.

The Abel transform does not recover refractivity structure within the interval. Recovery of refractivity within the interval must be derived via interpolation if the Abel transform approach is used. There are several constraints that can be applied to both the singularity and interpolation across the critical refraction interval. Constraints include $n$ and $r$ derived at the top of the interval via the transform of data taken above the interval plus the requirement that $\partial N / \partial r$ at the top of the interval equal the critical gradient, $-10^{6} \mathrm{Rc}$. At the bottom of the interval, constraints include the Abel transform results integrated across the singularity and $a_{0}$ $=r_{0} n_{0}=a_{1}$. The vertical gradient of refractivity is constrained such that its integral over the interval must equal $n_{1}-n_{0}$ to which one can add requirements of continuity at the boundaries and smoothness over the interval and that its structure explains the observed vertical extent of critical refraction. Given the small vertical extent of critical refraction intervals, interpolation across the interval should be accurate, limited primarily by the accuracy of the Abel integration across the singularity. The high-resolution profile of $\alpha(a)$ derived following backpropagation (Section 7) should improve the accuracy at and below the lower edge of the critical refraction interval and therefore improve the accuracy of the Abel transform across the interval.

Errors in derived refractivity due to misrepresentation of the singularity will be largest at the bottom of the critical refraction interval and will decrease rapidly at lower altitudes because the contribution of the critical refraction interval to total bending decreases dramatically as the tangent height descends (Fig. 4). The magnitude of the refractivity error is also limited by the fact that the retrieved heights must be monotonic. A large refractivity error will result in a radius which is out of sequence via (14) and therefore clearly in error.

The prototype GPS-MET receiver typically tracks to $5 \mathrm{~km}$ altitude or lower, indicating that critical refractivity gradients extending over sufficiently large vertical and horizontal scales to extinguish occulted GPS signals must be at or below $5 \mathrm{~km}$ altitude. Given the low gain of the GPS/MET antenna, it is likely that critical refraction regions lie still lower in altitude probably associated with the extreme gradients of capping inversions marking the transition between the boundary layer and the free troposphere at the trade wind inversion. Critical refraction is therefore probably limited to within $4 \mathrm{~km}$ (and more typically $2 \mathrm{~km}$ ) of the surface at low latitudes and lower altitudes at higher latitudes. Any degradation in accuracy associated with these extreme gradients beyond the accuracy expectations summarized in Section 10 will be limited to the altitude interval within a few kilometers of the surface.

\subsection{Signal-to-Noise Performance}

The GPS network transmits relatively powerful signals designed for simple, hand-held receivers. In the absence of atmospheric attenuation the prototype GPS-MET receiver with a small hemispherical field-of-view antenna in low Earth orbit has obtained SNRs of $\sim 1-2 \times$ 
$10^{3}$ over the $20 \mathrm{~ms}$ integration period used at present for the radio occultation observations. Future instrumentation will improve upon this SNR by perhaps an order of magnitude.

Allowing for the molecular oxygen attenuation factor of 2 near the surface and the SNR of 10 required for receiver phase lock, attenuation by a factor of at least $100(20 \mathrm{~dB})$ in intensity due to atmospheric defocusing can be tolerated before loss of signal occurs. This margin is sufficient for signals to be tracked throughout the atmosphere with the exception of regions of very sharp refractivity gradients (Fig. 5a). GPS signal paths will in fact probe the entire atmosphere apart from occasional small critical refraction intervals just discussed. Implications of SNR on the accuracy of derived refractivity, pressure, and temperature have been discussed by Kursinski et al. (1997).

Encryption of GPS signals for military purposes is another issue. Two types of encryption exist: selective availability (SA), which alters the apparent GPS satellite positions, and anti-spoofing (AS), which eliminates jamming of the GPS receivers by false signals. Methods for eliminating SA as part of the clock calibration process are discussed in Section 9. The AS encryption affects the L1 and L2 P-code signals (Section 2) and therefore calibration of the ionosphere. While a classified receiver with knowledge of the encryption code can recover the full precision of the signal, it is desirable to use a nonclassified cross-correlation approach which produces little degradation when SNRs are high such as that developed for the BlackJack receiver at JPL which will fly on the COSMIC constellation. The SNR of a cross-correlated signal decreases as the square of the atmospheric attenuation such that the factor of 40 attenuation due to molecular oxygen absorption and defocusing near the surface (Fig. 5a) will decrease the SNR of the cross-correlated signal by 1600 (32 dB). The BlackJack design will produce 1 second cross-correlation SNRs of the order of $47 \mathrm{~dB}$ or higher that are sufficient to overcome $\sim 18 \mathrm{~dB}$ of atmospheric attenuation and still achieve the required SNR of 10 to maintain phase lock. Therefore the ionosphere calibration can be maintained through most of the troposphere with unclassified instrumentation. Since errors due to incomplete calibration of the ionosphere are generally negligible in the lower troposphere (Kursinski et al., 1997), interpolation or extrapolation of the ionosphere calibration across the short vertical intervals in the troposphere where attenuation is too large to maintain cross-correlation racking should have negligible impact on retrieval accuracy.

\section{ATMOSPHERIC MULTIPATH}

Atmospheric multipath (AMP) refers to the situation where multiple ray paths through the atmosphere exist simultaneously and connect the same transmitter and receiver positions. Recovering all of the signals poses a serious challenge to the occultation receiver which is normally phased locked to a single signal.

\subsection{Conditions for Atmospheric Multipath}

AMP requires that the magnitude of the bending angle increase locally with altitude requiring that the magnitude of the refractivity gradient increase with altitude and $\mathrm{d}^{2} N / \mathrm{d} r^{2}<0$ in this local vertical interval. In terms of bending angle, the basic requirement for atmospheric 
multipath where ray paths cross at or before reaching the receiver can be written as

$$
\frac{d \alpha}{d a} \geq \frac{L_{t}+L_{r}}{L_{t} L_{r}} \simeq \frac{1}{L_{r}} .
$$

The approximation in (25) is true when the receiver is much closer to the limb than the transmitter as is the case for a LEO GPS receiver. In discrete form the AMP requirement is $\Delta \alpha \geq \Delta \mathrm{a} / L_{r} \sim \Delta \mathrm{r} / L_{r}$ where $\Delta \alpha$ is the difference between bending angles, $\alpha\left(\mathrm{r}_{1}\right)$ and $\alpha\left(\mathrm{r}_{2}\right)$ and $\Delta r=r_{1}-r_{2}$. A more restrictive definition of AMP requires that the multiple ray path tangent heights be separated by at least the diameter of the first Fresnel zone, $F$, in order that separate and distinct geometrical rays exist. In such a case, the $\alpha$ (a) profile derived from these rays will be invertable via the Abel transform. With $\mathrm{D}=3000 \mathrm{~km}$ and $\mathrm{F} \sim 1 \mathrm{~km}, \Delta \alpha \sim$ $3 \times 10^{-4}$ radians $=0.018$ degrees which is a small fraction of total bending angle in the troposphere implying multipath may be encountered frequently in the troposphere.

\subsection{Modeling Atmospheric Multipath due to Sharp Layers}

The most common cause of AMP at GPS wavelengths is probably sharp layers in moisture but it may also be caused by sharp temperature structure in the troposphere. A narrow layer of a high concentration of water vapor creates a sharply defined dipole in the vertical refractivity gradient that is superimposed on the approximately exponential refractivity gradient of the background atmosphere. A situation frequently encountered in the subtropics is the transition from a dry region to a moist region below, such as the transition between the free troposphere and the convective boundary layer below. In this geometry, a single spike in refractivity gradient at the transition is superimposed on the approximately exponential gradient of the background atmosphere. The spike in refractivity gradient produces a sharp peak in bending angle at the altitude of the spike which decreases rapidly at lower tangent altitudes because of the focused weighting of the $\left(r^{2} n^{2}-a^{2}\right)^{-1 / 2}$ term in (12) near the tangent altitude. The resulting AMP is limited to an interval at and just below the layer of high refractivity gradient.

Using simple scaling arguments one can determine approximately the effect of sharp transition layers on bending angle and the conditions of AMP. From (25), the requirement for AMP can be written as

$$
\alpha\left(z_{t}\right)-\alpha\left(z_{t}-\Delta z\right) \sim \frac{\Delta z}{L},
$$

where $z$, refers to the ray path tangent height in the transition layer with the high refractivity gradient and $z_{t}-\Delta z$ is some lower altitude where $\Delta z \geq F$. The bending at height, $z$, is the bending due to the background exponential structure of the atmosphere plus the contribution due to the high gradient in the transition layer:

$$
\alpha\left(z_{t}\right)=\alpha_{0}\left(z_{t}\right)+\delta \alpha_{t}\left(z_{t}\right) \equiv \alpha_{0}\left(z_{t}-\Delta z\right)+\delta \alpha_{t}\left(z_{t}-\Delta z\right)+\Delta z / L
$$

The 0 subscript refers to the bending in the absence of the transition layer and $\delta \alpha_{t}\left(z_{t}\right)$ and $\delta \alpha_{t}\left(z_{t}-\Delta z\right)$ are the bending contribution of the transition gradient for the ray paths with tangent heights, $z_{t}$ and $z_{t}-\Delta z$, respectively. The background bending is approximately expo- 
nential with altitude such that

$$
\alpha_{0}\left(z_{t}-\Delta z\right) \cong \alpha_{0}\left(z_{i}\right) e^{\Delta z / H_{\alpha}} .
$$

Combining (26) and (27) yields the extra bending from the transition layer required to produce AMP:

$$
\delta \alpha_{t}\left(z_{t}\right)-\delta \alpha_{t}\left(z_{t}-\Delta z\right) \cong \alpha_{0}\left(z_{t}-\Delta z\right)-\alpha_{0}\left(z_{t}\right)+\Delta z / L \cong \alpha_{0}\left(z_{t}\right)\left[e^{\Delta z / H_{\alpha}}-1\right]+\Delta z / L
$$

Because of the square root term in the denominator in $(11), \delta \alpha_{t}\left(z_{t}\right)$ and $\delta \alpha_{t}\left(z_{t}-\Delta z\right)$ are related approximately as

$$
\delta \alpha_{t}\left(z_{t}-\Delta z\right) \cong \delta \alpha_{t}\left(z_{t}\right)\left(\sqrt{\Delta z / \Delta z_{t}+1}-\sqrt{\Delta z / \Delta z_{t}}\right),
$$

where $\Delta z$ is the thickness of the transition layer. Combining (28) and (29) yields

$$
\delta \alpha_{t}\left(z_{t}\right)\left(\sqrt{\Delta z / \Delta z_{t}+1}+\sqrt{\Delta z / \Delta z_{t}}\right) \cong \alpha_{0}\left(z_{t}\right)\left[e^{\Delta z / H_{x}}-1\right]+\Delta z / L .
$$

Half of the bending occurs within a vertical interval, $\Delta z_{1 / 2}$, of the ray tangent height (Fig. 4). Furthermore, for a given refractivity gradient, the contribution of a given layer to the bending scales approximately as the square root of the layer thickness (derivable from (11)). Therefore, the total bending at $z_{\text {, }}$ can be written as

$$
\frac{\delta \alpha_{t}\left(z_{t}\right)}{\alpha_{0}\left(z_{t}\right)}=\frac{(\Delta d N / d r)_{t}}{(d N / d r)_{0}} \frac{1}{2}\left[\frac{\Delta z_{t}}{\Delta z_{1 / 2}}\right]^{1 / 2}
$$

where ( $\Delta d N / d r)$, is the extra refractivity gradient contributed by the transition layer and $(d N /$ $d r)_{0}$ is the refractivity gradient at $z_{t}$ contributed by the exponential refractivity behavior. Combining (30) and (31) yields

$$
e^{\Delta z / H \alpha-1} \simeq \frac{\Delta z}{H_{\alpha}} \simeq \frac{(\Delta d N / d r)_{t}}{2(d N / d r)_{0}}\left[\frac{\Delta z_{t}}{\Delta z_{1 / 2}}\right]^{1 / 2}\left(1-\left[\frac{\Delta z}{\Delta z_{t}}+1\right]^{1 / 2}+\left[\frac{\Delta z}{\Delta z_{t}}\right]^{1 / 2}\right)-\frac{\Delta z}{L \alpha_{0}\left(z_{t}\right)},
$$

and the requirement on the incremental increase in refractivity gradient at the transition layer is

$$
\left(\Delta \frac{d N}{d r}\right)_{t} \simeq 2\left(\frac{d N}{d r}\right)_{0} \Delta z\left[\frac{1}{H_{\alpha}}+\frac{1}{L \alpha_{0}\left(z_{t}\right)}\right]\left[\frac{\Delta z_{1 / 2}}{\Delta z_{t}}\right]^{1 / 2}\left(1-\left[\frac{\Delta z}{\Delta z_{t}}+1\right]^{1 / 2}+\left[\frac{\Delta z}{\Delta z_{t}}\right]^{1 / 2}\right)^{-1}
$$

In the troposphere, bending is generally large enough that the $L \alpha_{0}$ term can be ignored to first order. With representative tropospheric values of $\Delta z=F \sim 0.8 \mathrm{~km}, \Delta z_{1 / 2}=1.6 \mathrm{~km}$ (Fig. 4), $H_{\alpha}=6 \mathrm{~km}$ and $\Delta z_{t}=0.1 \mathrm{~km},(32)$ requires the additional refractivity gradient of the layer needed to produce AMP with distinctly separated ray paths must be at least 1.4 times the background atmosphere gradient. 
The fact that the $H_{\alpha}^{-1}$ term dominates the $\left(L \alpha_{0}\right)^{-1}$ term in (32) indicates that the primary obstacle in causing rays to cross at the distance of a LEO receiver is overcoming the exponentially increasing bending angles with decreasing height due to the background atmosphere. When backpropagation is performed to reduce the effects of diffraction, this is no longer true and the $\left(L \alpha_{0}\right)^{-1}$ term becomes dominant (see Section 7$)$.

\subsection{Atmospheric Multipath due to a Thermal Inversion}

The large refractivity gradient needed to create AMP can be provided by a large positive temperature gradient at a thermal inversion. The density scale height, $H_{\rho},\left(=H_{N}\right.$, the refractivity scale height, in dry air) is given by

$$
H_{\rho}=\frac{H_{p}}{\left(1-H_{p} \frac{d T}{T d z}\right)},
$$

where $H_{P}$ is the pressure scale height. Since the refractivity gradient is $-N / H_{N}$, increasing the gradient magnitude requires decreasing $H_{N}$ which in turn requires that the inversion temperature gradient, $(d T / d z)_{t}$, be related to the background temperature gradient, $(d T / d z)_{0}$, as

$$
\left(\frac{d T}{d z}\right)_{t}=\left(\frac{T}{H_{p}}+\left(\frac{d T}{d z}\right)_{0}\right) \frac{H_{N 0}}{H_{N t}}-\frac{T}{H_{p}} .
$$

The strong tradewind inversion across the subtropics provides a case where AMP will occur. For ATEX, the average inversion thickness was $\sim 200 \mathrm{~m}$ and $Z_{\mathrm{F}}$ is $\sim 0.6 \mathrm{~km}$ in the lower troposphere where the inversion exists (see Fig. 5b). Under these conditions, (31) indicates atmospheric multipath will occur when an additional refractivity gradient greater than 0.9 times that of the background atmosphere exists. The temperature gradient at the inversion required to achieve AMP according to (33) is $+19 \mathrm{~K} / \mathrm{km}$. Since the average temperature gradient across the tradewind inversion observed during ATEX was $+25 \mathrm{~K} / \mathrm{km}$, geometric multipath should occur systematically at the tradewind inversion. The humidity contribution will further enhance the refractivity gradient at the inversion. The tradewind results suggest that thermal inversions overlying relatively cold surfaces will provide conditions where AMP can be expected to occur. Other than these types of conditions, it will be difficult in general to find temperature gradients large enough to create AMP. Notice that the conditions necessary for atmospheric multipath are easier to meet than the critical refraction conditions (see Section 5) which are not met by average ATEX temperature inversion.

\subsection{Atmospheric Multipath due to a Humidity Gradient}

Creating AMP with a water vapor layer requires that the concentration of the vapor be large enough and vary rapidly enough with altitude to meet the requirement of (32). The extreme situation of $100 \%$ relative humidity below the layer and $0 \%$ above establishes the minimum temperature at which geometric AMP can be caused by water vapor for a given layer thickness: 


$$
P_{w}^{*}=2 \frac{T^{2} N}{b_{2} H_{N}} \Delta z\left[\frac{1}{H_{N}}+\frac{1}{L \alpha_{0}\left(z_{t}\right)}\right]\left[\Delta z_{t} \Delta z_{1 / 2}\right]^{1 / 2}\left(1-\left[\frac{\Delta z}{\Delta z_{t}}+1\right]^{1 / 2}+\left[\frac{\Delta z}{\Delta z_{t}}\right]^{1 / 2}\right)^{-1},
$$

where $P_{w}{ }^{*}$ is the saturation vapor pressure. Figures $6 \mathrm{a}, 6 \mathrm{~b}$ and $6 \mathrm{c}$ show the pressure (or equivalently altitude) dependence of (34) for the equator, $30^{\circ}$ and $60^{\circ}$ latitude respectively based on the annual climatology of Peixoto and Oort (1992). The results reflect the dependence of (34) on height for a $100 \mathrm{~m}$ thick transition layer and the ray path tangent heights are separated vertically by F, the diameter of the first Fresnel zone. The solid line in each figure is the saturation vapor pressure according to the annual climatology of Peixoto and Oort (1992). The altitude at which the solid line crosses the $\mathrm{L}=3000 \mathrm{~km}$ line is the maximum altitude where multipath may occur according to (34). The other lines are discussed in Section 7. The maximum height at the equator is $\sim 10 \mathrm{~km}$ and decreases to $\sim 6 \mathrm{~km}$ at $60^{\circ}$. The maximum height of AMP at the equator is $-5 \mathrm{~km}$ higher than the maximum height of critical refraction. The general conclusion to be drawn is that multipath may be encountered frequently in the lower

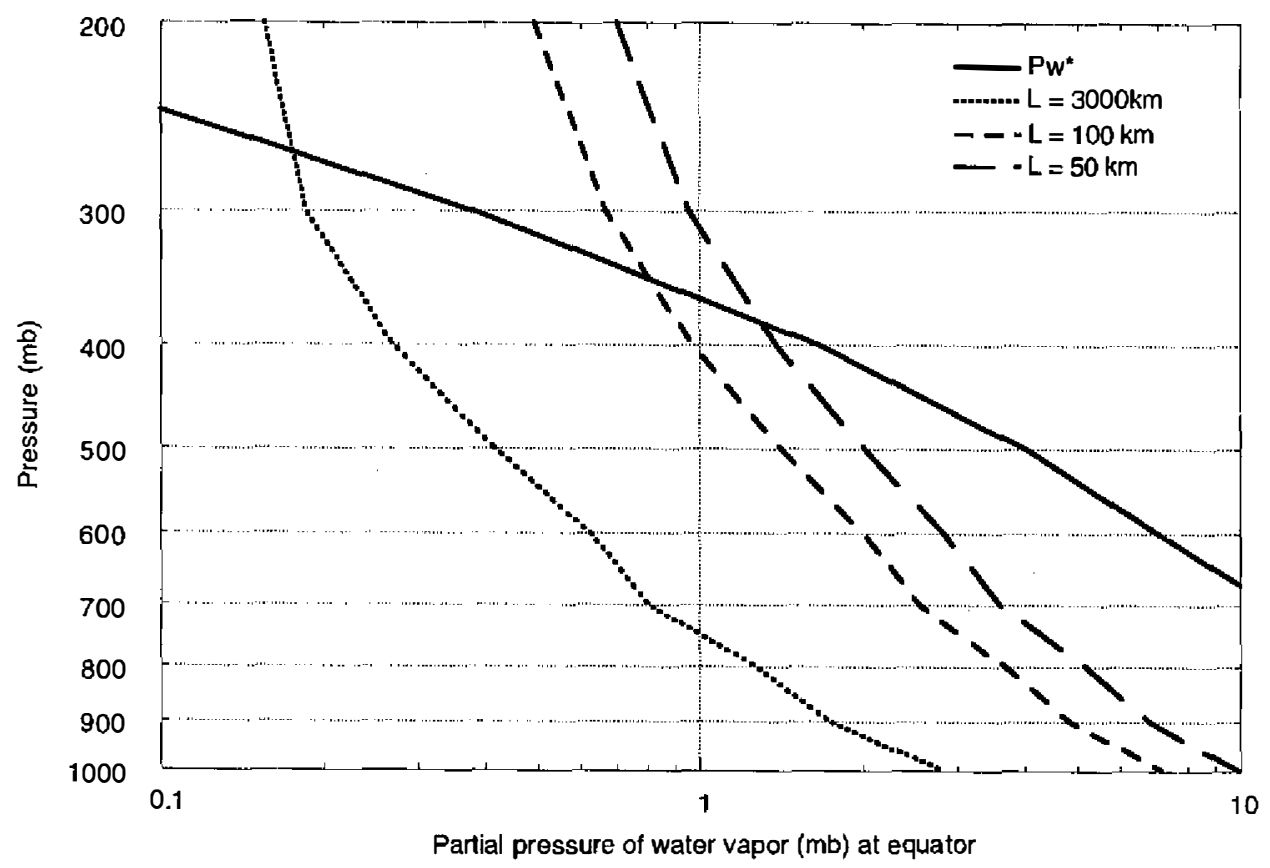

Fig. 6. Maximum height of atmospheric multipath assuming a transition layer $100 \mathrm{~m}$ thick with $0 \%$ relative humidity above the layer and $100 \%$ relative humidity below the layer based on (34). Solid line: climatological saturation vapor pressure of water vapor. Dotted line: receiver in LEO located $3000 \mathrm{~km}$ from the limb. Dashed lines: two backpropagation results at $100 \mathrm{~km}$ and $50 \mathrm{~km}$ from the limb. (a). Equatorial conditions. (b). $30^{\circ}$ latitude conditions. (c). $60^{\circ}$ latitude conditions. Conditions are based on the Peixoto and Oort (1992) annual mean climatology. 


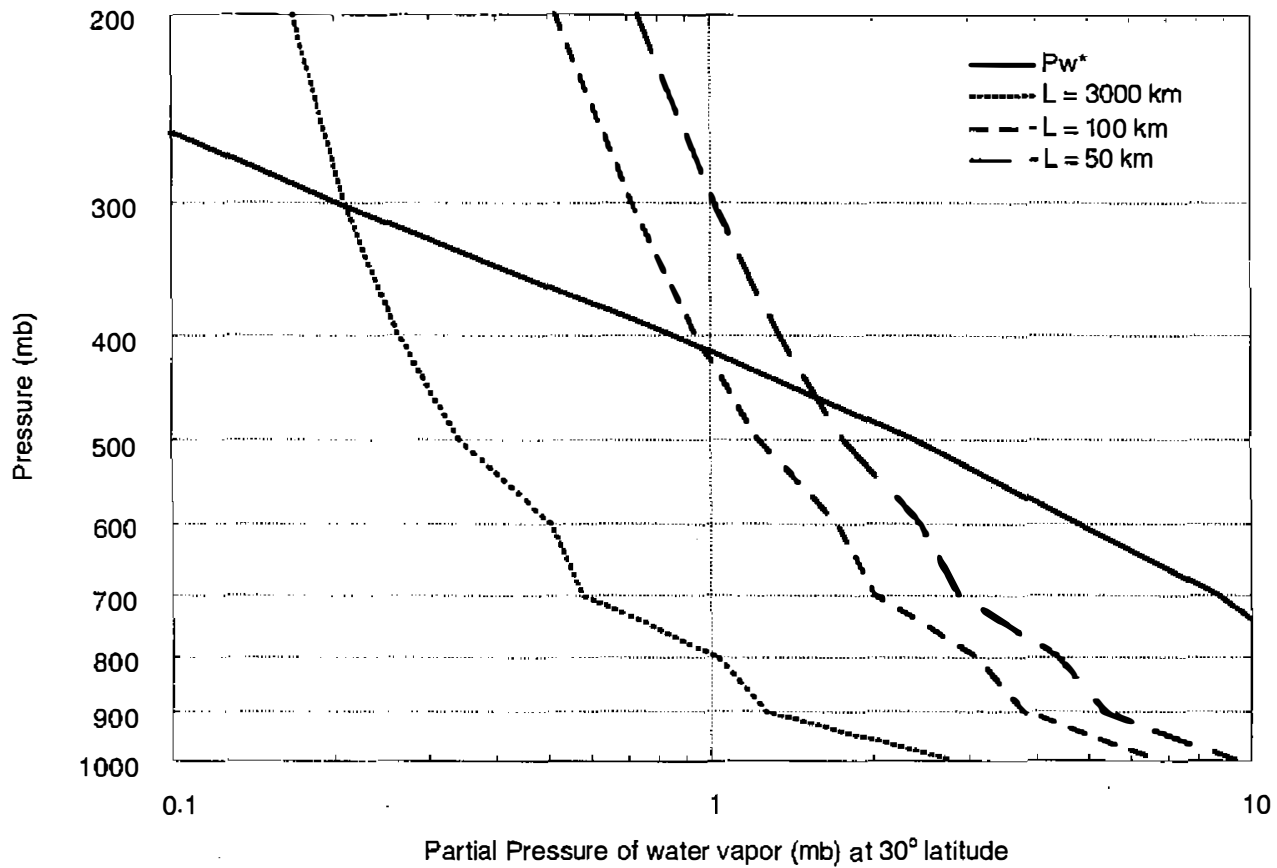

(b)

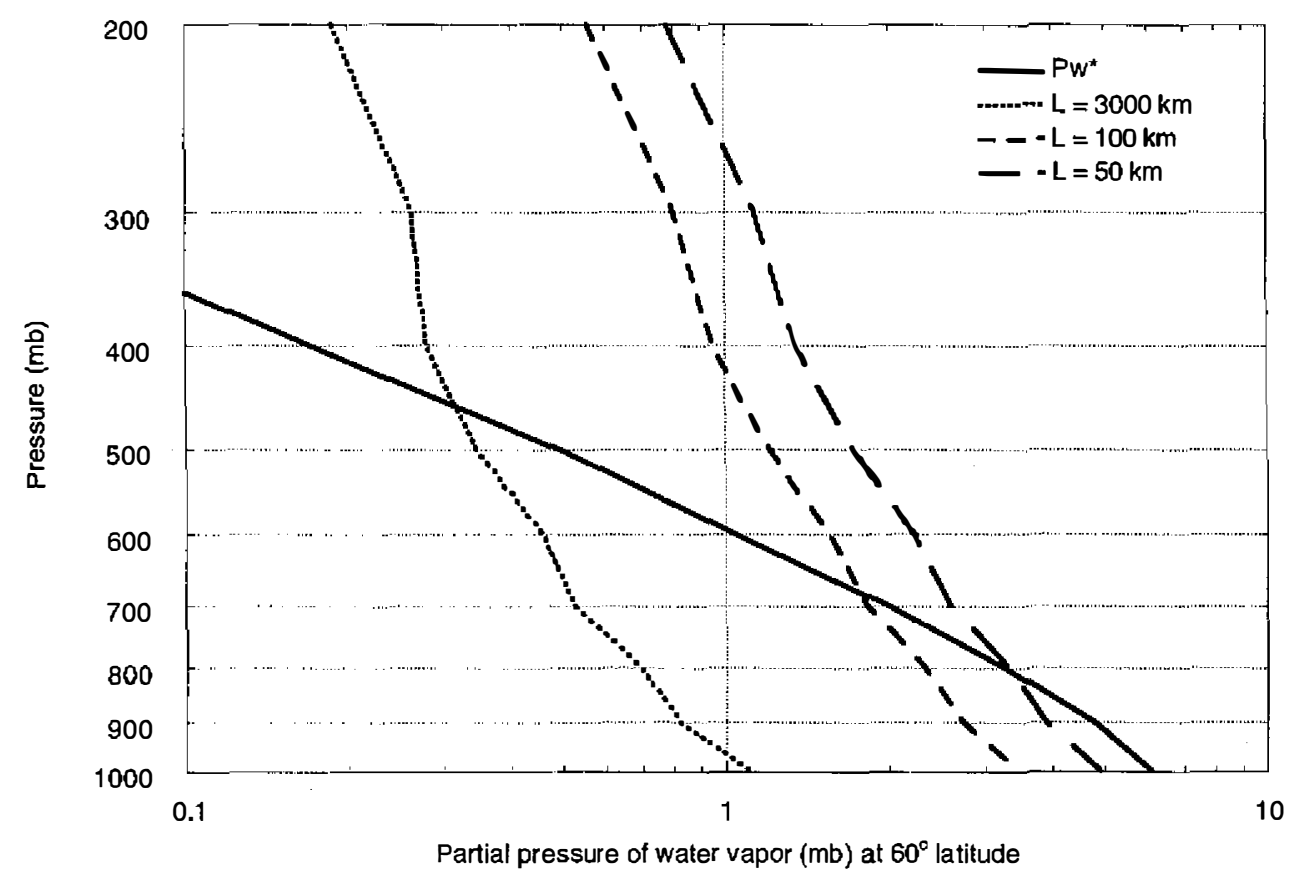

(c)

(Fig. 6. continued) 
troposphere.

\subsection{AMP Signal Acquisition and Retrievals}

It is important to realize that given spherical symmetry, even when ray paths cross, each path has a unique asymptotic miss distance, $a$, such that the tangent height of each ray path is unique via (14). Since $a$ defines the angle at which the ray hits the receiver via (8), each path must have a unique Doppler shift such that each signal and its corresponding $\alpha$ and $a$ can be separated according to its Doppler frequency. Therefore if the multipath signals are well defined in a geometrical optics sense, a Fourier transform of the received signal spectrum will yield distinct spectral peaks, each one associated with a particular raypath. Such an approach was used to separate and detect the frequencies of multipath signals associated with methane cloud decks in the Voyager occultations of Uranus and Neptune (Lindal et al., 1987; Lindal, 1992).

It follows that every ray path must be recovered to properly derive the refractivity near the tangent height of each raypath. The receiver must simultaneously track and recover all the images of the transmitted GPS signal present during multipath. To capture the multiple signals the acquisition bandwidth of the receiver must be sufficiently wide to satisfy the Nyquist criterion regarding the separation of the Doppler frequencies of the simultaneous multipath signals.

The Fourier transform approach for determining the Doppler frequencies is limited by diffraction effects that blur the Doppler frequency spectrum and can make identification of the individual signals difficult. Backpropagation as discussed in Section 7 both reduces the occurrence of multipath and reduces diffractive effects which sharpens the signal spectrum and improves signal detection via a Fourier transform approach.

Another complication when AMP occurs is the ray path tangent height no longer decreases or increases monotonically with time and the resulting time sequence of $\alpha(a)$ will not be in monotonically descending order of $a$. In fact, the last signal observable in a setting occultation may be a signal whose ray path tangent height is well above the surface such as at the tradewind inversion. Therefore, the $\alpha(a)$ sequence must be sorted into a monotonically ascending or descending order of a as required to calculate the Abel transform (13).

Nonspherical structure complicates interpretation of the observed Doppler spectrum because the assertion that $\boldsymbol{\alpha}, \alpha$ and the corresponding Doppler frequency are unique will not be true in the presence of significant horizontal structure. Dealing with nonspherical structure is an ongoing area of research.

\section{REDUCING THE EFFECTS OF MULTIPATH AND DIFFRACTION: THE BACKPROPAGATION CONCEPT}

\subsection{Motivation for Reducing the Effects of Diff raction}

The vertical resolution of the GPS occultations is quite good for space-bome remote sensing. Still better resolution is desirable for recovering fine vertical scale structure where it 
exists. A good example is the planetary boundary layer (PBL) which the GPS wavelengths can routinely penetrate unlike shorter wavelengths. The depth of the PBL ranges typically from 100 meters to 2 kilometers. GPS resolution in the lower troposphere tends to be roughly 500 meters (Fig. 5b). Resolution is better on the topside of the inversions that often cap the PBL but worse on the inversion's bottom side due to the focusing there. Therefore the raw GPS occultation resolution is well suited to characterize the PBL top but has limited ability to characterize the PBL interior. Improving vertical resolution would therefore be quite useful for characterizing the interior structure of the boundary layer from space.

Another very important issue is multipath which was discussed in Section 6. Dealing with multipath presents a serious challenge to the occultation receiver because the receiver must track multiple images of the same signal simultaneously. Assuming the full signal spectrum is recovered by the receiver, and atmospheric structures are larger than the Fresnel size such that the geometric optics approximation is valid, the multiple signals can be separated and tracked using a Fourier transform-type approach and a full refractivity profile derived. When diffraction becomes important, i.e., structures smaller than the Fresnel size are encountered, electromagnetic propagation becomes more complicated than raypath optics, and a more complex analysis approach is required.

\subsection{Overview of the Backpropagation Concept}

Exceeding the vertical resolution imposed by Fresnel diffraction and the observation geometry described in Section 4 and undoing the ray crossings associated with atmospheric multipath described in Section 6 are possible because the signals are essentially monochromatic with well-defined phase and amplitude. The radio occultation preserves phase information. As in holography, a nearly complete reconstruction of the scalar electromagnetic field is possible given phase information on a plane. To the extent that the atmosphere can be represented as a one-dimensional, thin diffracting screen, the one-dimensional slice of the receiver's orbit through the resulting diffraction pattern is sufficient to derive the properties of the diffracting screen. The idea is that given the full recovery of the occulted signal's complex phase and amplitude along the receiver's path, the signal can be "back-propagated" through the vacuum between the receiver and the atmosphere to a location much closer to the atmosphere. Back-propagating the signal yields two important improvements. First, vertical resolution is improved because the reduced distance between the limb and the apparent receiver reduces the Fresnel zone size (see (21) and (22)). Secondly, by reducing the distance between the atmosphere and receiver, we move the receiver in front of the region where the rays cross such that only one ray path is associated with each location along the back propagation line which simplifies interpretation of the complex phase and amplitude.

The back-propagation concept was originally developed by Marouf et al. (1986) at Stanford University to dramatically reduce the effects of diffraction in Voyager occultation observations of the Saturnian and Uranian ring systems and improve radial resolution relative to diffraction-limited scales. Reduction of diffraction effects due to an atmosphere is more complicated because the atmosphere is a three-dimensional structure. Two essentially equivalent approaches for reducing atmospheric diffraction effects via 2D back-propagation have been 
developed (Gorbunov et al., 1996 and Karayel and Hinson, 1997). The beauty of such an approach is that it assumes very little about the atmospheric structure which caused the observed diffraction pattern and simply propagates the signal backwards through the intervening vacuum between the receiver's orbit and a set of positions much closer to the atmosphere.

\subsection{Atmospheric Backpropagation in 2D}

The light propagation through the atmosphere and the resulting diffraction effects depend on all 3 spatial dimensions in general. However, since the typical scale of horizontal variations in the atmosphere are large in comparison to the Fresnel scale $(\sim 1 \mathrm{~km})$, the atmospheric occultation diffraction problem can be reduced to a 2 dimensional form in the occultation plane (Gorbunov et al., 1996). The large argument expansion of the Green's function of the $2 \mathrm{D}$ wave equation is

$$
G(r)=\frac{1}{4 i}\left(\frac{2}{\pi k r}\right)^{1 / 2} \exp (i k r-i \pi / 4),
$$

where $k$ is the wave vector $=2 \pi / \lambda$ and $r$ is the distance from the origin. Based on (35), Gorbunov et al. showed that the relationship between the source complex amplitude, $u(y)$, along a straight line, $S y$, and the resulting complex amplitude $u(x)$ at some point, $x$, which lies off the line is

$$
u(x)=\left(\frac{k}{2 \pi}\right)^{1 / 2} \int_{s_{y}} u(y) \cos \left(\psi_{x y}\right) \frac{\exp (i k|x-y|-i \pi / 4)}{|x-y|^{1 / 2}} d S_{y},
$$

where $\psi_{x y}$ is the angle between the normal to the line, $S_{y}$, and the vector, $x-y$. If the complex amplitude is measured along another line, $S_{x}$, then the complex amplitude along the line, $S_{y}$, can be derived as follows

$$
u(y)=\left(\frac{k}{2 \pi}\right)^{1 / 2} \int_{s_{x}} u(x) \cos \left(\psi_{x y}\right) \frac{\exp (-i k|x-y|+i \pi / 4)}{|x-y|^{1 / 2}} d S_{x} .
$$

(36) and (37) represent a transform pair relating the complex signal amplitudes along the two lines in the occultation plane (see Fig. 7). Note that the change of sign of the terms in the exponential in the second equation represents propagation in the direction opposite to that in the first equation. Given a constant index of refraction in the medium containing the two lines and the area between them, (37) allows the signal measured along the receiver's trajectory to be numerically propagated backwards to a line much closer to the Earth's limb.

As discussed by Gorbunov et al., the most significant contribution of the integral in (37) comes from the region near the point of stationary or minimum phase of the integrand where phase is defined as

$$
\Phi=\arg (u(x))-k \mid x-y,
$$

Therefore, for each point, $y$, on the backpropagation line, the point, $x$, on the LEO orbit for which $\Phi$ is minimum is the point about which the integration limits are centered. Gorbunov et al. found that integration limits which span $\pm 10 \pi$ of phase centered on the $x$ of minimum 


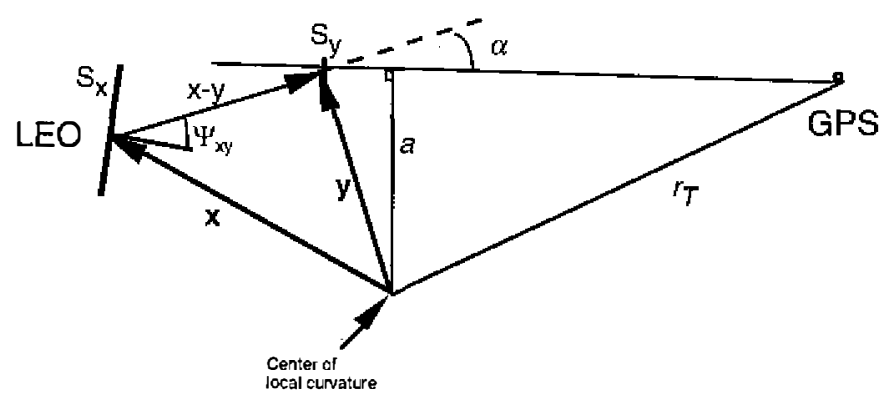

Fig. 7. Occultation geometry in the occultation plane showing the line of the LEO orbit, $S_{x}$, and the backpropagation line, $S_{y}$.

phase work as well as wider integration limits in general.

Since the diffraction transform pair, (36) and (37), is in 2D whereas the observations are in $3 \mathrm{D}$, a 3D to $2 \mathrm{D}$ coordinate transformation is required. The $2 \mathrm{D}$ occultation plane is defined approximately as that which contains the transmitter, receiver and the vector normal to the geoid at the tangent point of the ray path connecting the transmitter and receiver (see Section 3.5). This plane is an approximation because the ray path is not exactly planar due to refractivity gradients orthogonal to that plane (10), which tend to be maximum when large amounts of water vapor are present. Because the transmitter and receiver lie in different orbital planes the occultation plane changes continuously during an occultation.

\subsection{Transmitter Motion during the Occultation}

The transmitter's motion during the occultation must also be considered because relative motion between the transmitter and atmosphere contributes to the complex amplitude measured by the receiver which is not accounted for by the diffraction transform pair, (36) and (37). When establishing the transformation, the position of the transmitter is typically fixed. For a diffraction integral to work rigorously, the atmosphere-transmitter orientation should not change. If we consider the atmosphere as spherically symmetric, this means that the distance between the transmitter and the center of curvature should not change. Any deviation is referred to as "transmitter motion" in diffraction calculations. If the distance, $r_{r}$, between the transmitter and center of curvature were fixed during the occultation, then, with the appropriate rotation, the transmitter location in the occultation plane could be fixed with respect to the center of curvature. However, the difference between the center of curvature and center of the GPS orbits causes $r_{T}$ to change by many GPS wavelengths over an occultation. A coordinate transformation is therefore needed which holds the transmitter position fixed in the occultation plane over the observation period.

Here we describe a slight improvement on the translation suggested by Gorbunov et al. (1996) based on geometric optics. The concept is to adjust the transmitter position such that $\mathrm{r}_{\mathrm{T}}$ remains fixed during an occultation by sliding the transmitter position along the ray path asymptote at the transmitter. Defining $r_{T 0}$ as the value at which to fix $r_{T}$ during the occultation, the translation, $\Delta s_{T}$, of the transmitter's position along the asymptote at time, $t$, is given as 


$$
\Delta s_{T}(t)=\frac{r_{T}(t)-r_{T 0}}{\cos \phi_{T}(t)}
$$

assuming $\Delta s_{T} \ll<r_{T}$ where $\phi_{T}(t)$ is defined by $a(t)=r_{T}(\mathrm{t}) \sin \phi_{T}(\mathrm{t})$. To hold the transmitter to receiver propagation delay along the ray path constant, the receiver's position must be translated by the same amount, $\Delta s_{T}(t)$, along the ray path asymptote at the receiving end (see Fig. 8). As a result, the receiver's position in the occultation plane is shifted from $\left(r_{R}, \theta_{R}\right)$ to $\left(r_{R^{\prime}}, \theta_{R^{\prime}}\right)$ defined as

$$
\begin{gathered}
r_{R^{\prime}}(t)=r_{R}(t)+\Delta s_{T}(t) \cos \phi_{R}(t), \\
\theta_{R^{\prime}}(t)=\theta_{R}(t)+\frac{\Delta s_{T}(t) \sin \phi_{R}(t)}{r_{R}(t)},
\end{gathered}
$$

where $\Delta s_{T}$ is defined in (38) and is assumed to be much less than $r_{R}$ and $\phi_{R}(t)$ is defined by $a(t)=r_{R}(t) \sin \phi_{R}(t)$. By translating the transmitter and receiver positions along the ray path, the bending angle, asymptotic miss distance and propagation delay between the transmitter and receiver remain constant. While similar, the Gorbunov et al. approach does not preserve propagation delay. Once the translation defined in (39) is made, an additional coordinate rotation is done in order to hold the transmitter position fixed in the occultation plane during the occultation. The translation along the raypath uses the bending angle and asymptotic miss distance that can be estimated prior to the back-propagation transform. If necessary, the bending angle and asymptotic miss distance can be re-estimated following backpropagation and the backpropagation repeated until convergence is achieved.

While suitable for an individual ray optics solution, the position translation does not account for all the paths that contribute to the Fresnel integral. In fact, a unique geometrical optics translation does not exist when there is multipath, that is, when multiple ray paths simultaneously connect a particular transmitter and receiver location. We can evaluate the adequacy of a single translation's ability to compensate for the motion of the transmitter for the

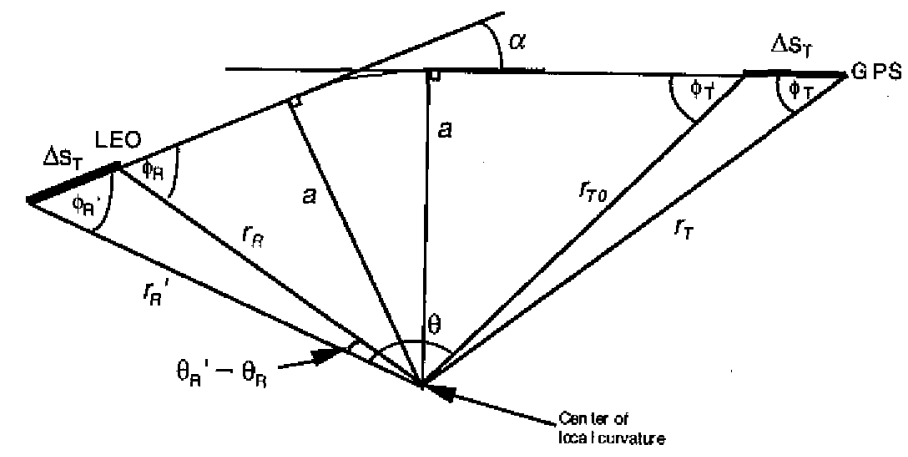

Fig. 8. Occultation geometry showing the translation of the transmitter and receiver positions along their respective ray asymptotes needed to preserve the distance between the transmitter and center of curvature during the backpropagation process. 
multiple signals with the following example. Consider the situation where two simultaneous raypaths exist in the lower troposphere with asymptotic miss distances of $a_{1}$ and $a_{2}$ separated vertically at their tangent points by $\sim 4 \mathrm{~km}$ such that $a_{1}-a_{2} \sim 4 \mathrm{~km}$. If we want the integral in (37) to include contributions of the first $\pm 50 \pi$ or Fresnel zones centered on the phase minimum, the corresponding vertical raypath motion must span roughly $7 F$ or $\sim 10 \mathrm{~km}$ at middle atmosphere heights based on (21). $10 \mathrm{~km}$ of vertical motion takes roughly 4 seconds given a tangent height descent rate of $2.5 \mathrm{~km} / \mathrm{sec}$ in the middle atmosphere. In the lower troposphere with the $\mathrm{M}$ factor in (18) equal to 50 , the time to descend $7 F$ is roughly 7 times the middle atmosphere time (see Section 9) or $\sim 30$ seconds. Given a circular GPS orbit and a center of curvature which differs from the center of the GPS orbit by $\sim 40 \mathrm{~km}$ due to oblateness, the GPS satellite distance from the center of curvature can change by $\sim 200 \mathrm{~m}$ over 30 seconds. The corresponding difference in the receiver translation along raypaths, $a_{1}$ and $a_{2}$, defined by (39) to compensate for the transmitter's $200 \mathrm{~m}$ radial shift will differ radially by roughly 0.2 meters which is quite small. Therefore a single translation works quite well because the GPS transmitter orbit is nearly circular, the GPS to limb distance is large and the multiple raypaths are sufficiently similar under multipath conditions.

\subsection{Derivation of $\alpha(\mathrm{A})$ from Backpropagated Signals}

We must now derive bending angles and asymptotic miss distances from the back-propagated complex amplitude as input to the Abel transform or a more general retrieval method. As pointed out by Karayel and Hinson (1997), the bending angle can be derived from the tilt of the phase of the complex amplitude along the back-propagation line, $S_{x}$, because light propagates in the direction normal to the surface of constant phase. The relations derived here differ slightly from those of Karayel and Hinson because of the finite distance between the GPS transmitter and center of curvature. As defined in Fig. 9, the angle, $\delta$, between the direction of propagation at point y on $S_{y}$ and the normal to $S_{y}$ is given as

$$
\delta=\sin ^{-1}\left(-\frac{\lambda}{2 \pi} \frac{d \Phi}{d z}\right)
$$

where $\lambda$ is the signal wavelength, $\Phi$ is the phase of the back-propagated signal in radians and $z$ is distance along $S_{y}$. The angle, $\phi_{B}$, between the propagation direction and the vector, $y$, defined as the vector between the center of curvature and the point $y$ on $S_{y}$ is given by

$$
\phi_{B}=\varepsilon+\delta,
$$

where $\varepsilon$ is the angle between the normal to $S_{y}$ and $y$. We therefore know the asymptotic miss distance by Bouguer's rule which is given as

$$
a=r_{B} \sin \phi_{B}=r_{T} \sin \phi_{T},
$$

where $r_{B}$ is $|y|$ and $\phi_{T}$ is the angle analogous to $\phi_{B}$ at the transmitter. To determine $\phi_{T}$ we must determine the $r_{T}$ that corresponds to $\mathbf{y}$ and $a$ in (42). To do so we extend the signal path forward from the point y along the asymptote defined by $a$ and $\phi_{B^{\prime}}$. The point where the path intersects the LEO receiver's orbit defines the receiver's position and the time $t(a)$ when the signal originally arrived at the receiver. Knowing the time of reception also determines the 


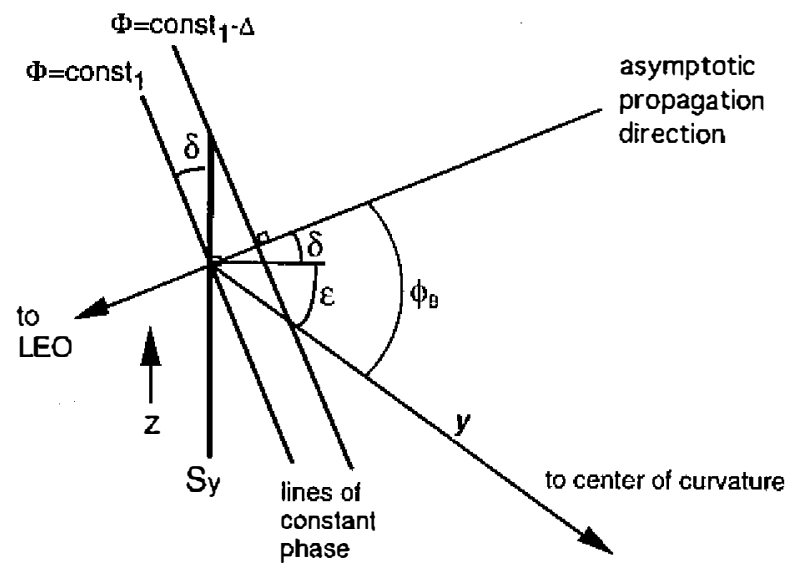

Fig. 9. Geometry at the backpropagation line, $S_{y}$, defining the angles needed to derive the asymptotic miss distance and the bending angle from the back propagated complex amplitude.

transmitter's position, $\mathbf{r}_{\mathrm{T}}$, at the transmission time, $f(a)$ minus the light travel time. $\phi_{T}$ is then found from $a$ and $r_{T}=\left|\mathbf{r}_{\mathbf{T}}\right|$ via (42). Given $\phi_{B}$ and $\phi_{T}$, the bending angle is found via

$$
\alpha=\theta+\phi_{T}+\phi_{B}-\pi
$$

where $\theta$ is the angle between $\mathbf{r}_{T}$ and $\mathbf{y}$.

\subsection{Estimates of Improved Resolution}

2D simulations have been performed to evaluate the backpropagation technique. In these simulations, the propagation of the signal through a model atmosphere is approximated, the back propagation is then applied and the recovered structure in the atmosphere is compared with the original structure to assess how well the backpropagation worked.

Three methods, all of which are 2D, have been employed to approximate forward propagation through the atmosphere. The first and simplest method is to collapse the phase delay associated with the atmosphere onto a single thin phase screen where the phase delay through the screen approximately represents that through the atmosphere. A more general approach used by Karayel and Hinson (1997) is to represent the atmosphere as a series of phase screens where each screen contains the phase delay associated with a portion of the atmosphere. Signal propagation between screens is accomplished using Fourier optics and a constant intervening refractivity. Mortensen et al. (1999) used the forward propagation concept described by Kursinski (1997) which combines ray optics within the Fresnel integral. The phase and amplitude of the transmitted signal along a line within the 2D atmosphere is determined based on the path lengths and defocusing respectively of ray paths connecting the transmitter to the plane. The propagation of the signal from the line within the atmosphere to the LEO receiver is accomplished by replacing the straight line $k r$ and $r^{-1 / 2}$ terms in the Fresnel integral (35) with the path lengths and defocusing of ray paths (see (18)) connecting the line to the receiver. Multiple atmospheric lines can be used.

The simulated 2D forward and backward propagation results of Karayel and Hinson (1997) and Mortensen et al. (1999) indicate that back-propagation reduces diffraction effects and 
resolves structures of order $250 \mathrm{~m}$ in vertical extent with an optimum location of the backpropagation line lying roughly 50-100 kilometers from the straight line tangent point. As pointed out by Karayel and Hinson, the optimal location lies well within the atmosphere beyond the region where the vacuum back-propagation should apply. Therefore the derived field does not represent the complex signal amplitude one would actually measure along the back propagation line. However, since the desired result is a set of high resolution estimates of bending angle versus asymptotic miss distance, the vacuum back-propagation technique provides the desired quantity, namely the complex amplitude along the ray path asymptote projected back well into the atmosphere.

The theoretical improvement in resolution defined by (22) depends on the reduction in $L_{R}$ in (21) and the $M$ factor in (22) defined in (18). Figure 10 shows the vertical resolution of the raw observations and results backpropagated to $L_{R}=50 \mathrm{~km}$. Note that the backpropagation resolution varies little with altitude because $M$ is nearly 1 along the backpropagation line. This is in contrast to the resolution of the original observations which varied by a factor of 7 due to the variations in $M$. The back propagation resolution is $\sim 200 \mathrm{~m}$ similar to the results of Karayel and Hinson (1997) and Mortensen et al. (1999). The increased resolution is particularly significant for sounding the planetary boundary layer (PBL) given the small vertical extent of the PBL.

It is important to note that the resolution results have all been generated in 2D with little if any horizontal structure. Horizontal variations in the height of a real 3D structure may limit the vertical resolution to be larger than $200 \mathrm{~m}$. As pointed out by Mortensen et al. (1999), the small horizontal variations associated with synoptic scale variations in the dry upper troposphere should not limit the vertical resolution. However, since water vapor of ten dominates

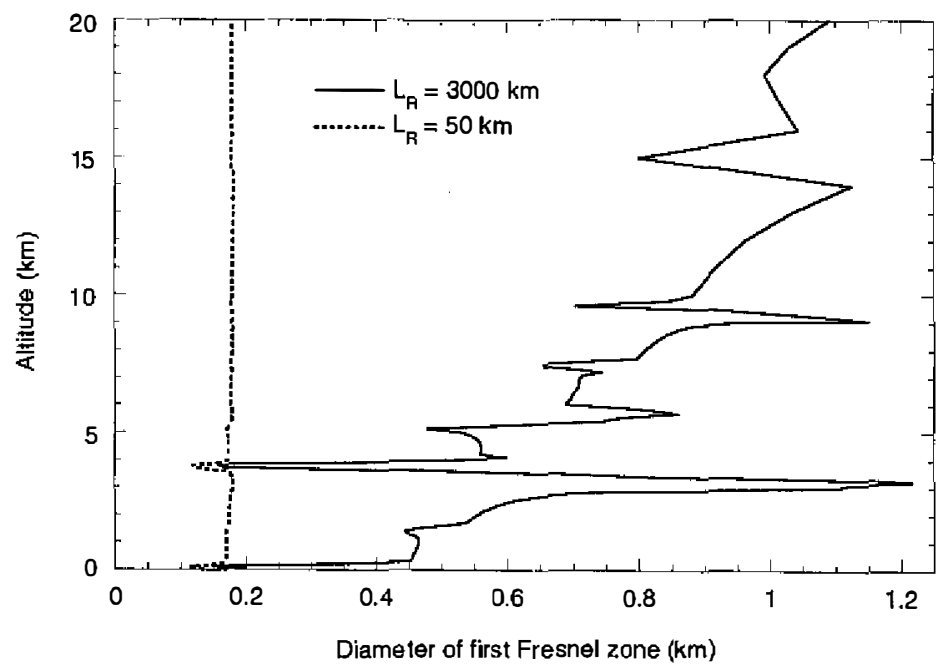

Fig. 10. Theoretical improvement in diffraction limited resolution with backpropagation. Solid line is resolution from figure $5 \mathrm{~b}$. Dashed line is theoretical resolution based on (21) and (22) after backpropagation to 50 $\mathrm{km}$ from the limb. 
the variability in the lower troposphere and given the small scales over which water vapor varies, it is likely that horizontal variations will limit the vertical resolution achievable within the PBL.

\subsection{Reduction of Multipath}

By moving the point of reception closer to the limb and dramatically reducing $L_{R}$, backpropagation can move the point of reception to a point where the rays have not yet crossed. Ray path crossings that occur still closer to the limb than the backpropagation line at $L_{R}$ will still exist in the backpropagation data. This is represented in (25) where the reduction in $L_{R}$ from that of the LEO $\left(=L_{R 0}\right)$ to that of the backpropagation line $\left(=L_{R B}\right)$ increases the minimum bending angle gradient, $\mathrm{d} \alpha / \mathrm{d} \boldsymbol{a}_{\min }$, which is required to produce multipath from $1 / L_{R 0}$ to $1 / L_{R B}$.

Using (34) we can gain some insight into the change in maximum altitude at which multipath can occur. (34) is based upon a step change in refractivity across a thin transition layer superimposed on an otherwise exponentially increasing atmospheric refractivity. The air above the layer is dry and the air below is saturated and the refractivity gradient across the layer is sufficient to produce multipath. Figure 6 shows the altitude at which multipath can occur according to (34) for both raw LEO observations and following backpropagation for $0^{\circ}, 30^{\circ}$ and $60^{\circ}$ latitude. Figure 6 contains the saturation vapor pressure of the annual mean atmosphere and the saturation vapor pressure required to produce multipath for a layer $100 \mathrm{~m}$ thick and $L_{R}$ equal to 3000,100 and $50 \mathrm{~km}$. The pressure level at which each $L_{R}$ multipath line cross the annual mean saturation vapor pressure represents approximately the highest atmospheric level where multipath may occur. The results show that in the tropics, multipath may occur up to $10 \mathrm{~km}$ in the LEO observations and the altitude is reduced to $\sim 7.5 \mathrm{~km}$ with backpropagation to $L_{R}=50 \mathrm{~km}$. At $30^{\circ}$ latitude the altitude change is roughly $3 \mathrm{~km}$ from 9 to $6 \mathrm{~km}$ altitude and roughly $4 \mathrm{~km}$ from 6 to $2 \mathrm{~km}$ altitude at $60^{\circ}$ latitude.

The altitude reduction associated with back propagation is not as large as one might expect (based on the change from $L_{R 0}$ to $L_{R B}$ ) for two reasons. First, as described in (32) and (34), the existence of multipath in the raw LEO observations is not limited by the $1 / L \alpha_{0}$ term but rather the $1 / H_{\alpha}$ term because the required increase in bending with increasing altitude to produce the ray path crossings must overcome the exponential behavior of the background atmosphere. Therefore, while the backpropagation reduction in $L_{R}$ from $3000 \mathrm{~km}$ to $50-100$ $\mathrm{km}$ in (32) does increase the magnitude of $(\Delta d N / d r)_{1}$ in (32), it does so only in proportion to $H_{\alpha} / \alpha_{0} L_{R B}$ rather than $L_{R O} / L_{R B}$.

The second reason is backpropagation improves vertical resolution. As described in Section 6 that vertical resolution is relevant because the geometric optics constraint in the multipath criterion requires that the vertical separation, $\Delta z$, between the tangent points of the crossing raypaths be at least the diameter of the first Fresnel zone, $F$. As vertical resolution improves, multiple phase minima in the Fresnel integral become separable as distinct raypaths and one can separate multiple raypaths that are more finely spaced in the vertical dimension. Therefore the minimum $\Delta z(=F)$ in (32) and (34) decreases as $L_{R}$ is reduced via backpropagation (see (21) and (22)) which decreases the magnitude of $(\Delta \mathrm{d} N / \mathrm{d} r)_{\mathrm{t}}$ and raises the maximum altitude where multipath may occur. 
The first of these two effects is larger such that the net effect of backpropagation is the minimum $(\Delta \mathrm{d} N / \mathrm{d} r)_{\mathrm{t}}$ does increase and maximum altitude decreases between the raw observations and the backpropagation in Fig. 6 but perhaps by surprisingly little.

\subsection{Open Issues in Backpropagation}

In the preceding discussion, several approximations and assumptions have been made. These include the receiver's orbit which is not quite a straight line and geometric optics-based compensation for the motion of the transmitter. Both appear reasonable particularly given the relatively short integration limits in (37) required to perform the backpropagation. As mentioned horizontal structure can limit the achievable resolution. Because the receiver's orbit is a line, it was necessary to treat the atmospheric structure as essentially cylindrical with a welldefined occultation plane. As with the Abel transform, any non-planar behavior in the signal propagation will not be properly represented in the 2D signal propagation approximations in (35) and (36) and will be mis-mapped into vertical structure in the assumed occultation plane. Any post-backpropagation multipath in regions where $\mathrm{d} \alpha / \mathrm{d} a>1 / L_{R B}$ will have to be dealt with using a Fourier transform or equivalent type approach to separate the different ray paths (see Section 6) by their unique bending angles and phase rates along the backpropagation line. Another issue which has not yet been assessed is non-monochromaticity because the GPS signal spectrum is actually more than $1 \mathrm{MHz}$ wide because of the ranging modulation.

\section{DERIVATION OF ATMOSPHERIC PROPERTIES FROM REFRACTIVE INDEX PROFILES}

\subsection{The Dependence of Refractive Index on Atmospheric Properties}

The interaction of electromagnetic radiation with the molecules and electrons in an atmosphere affects the speed of light propagation. At microwave wavelengths, the real component of atmospheric refractive index, $n$, (defined as the speed of light in a vacuum divided by that in the medium) is due to four main sources. These are the number density of dry atmosphere molecules and atoms, $n_{d}$, water vapor molecules, $n_{w}$, free electrons in the ionosphere, $n_{e}$, and particulates (primarily liquid water), $n_{p}$. As mentioned in Section 3 , since the index of refraction is very near unity, it is commonly expressed in terms of refractivity, $N$, the non-unity portion of $n$, and written as

$$
N=(n-1) \times 10^{6}=\mathrm{c}_{1} n_{d}+\mathrm{c}_{2} n_{w^{\prime}}+\mathrm{c}_{3} n_{e}+\mathrm{c}_{4} n_{p} .
$$

The dry molecules have no permanent dipole moment and contribute to refractivity viatheir polarizability, i.e., the ability of an incident electric field to induce an electric dipole the molecules. The $n_{d}$ term represents the combined contributions from the non-polar constitữents which make up the bulk atmosphere namely molecular nitrogen and oxygen, argon and carbon dioxide. Although each molecule has a different refractivity scale factor, these terms can be grouped together into a single term in (44) because they are well mixed at altitudes below the 
homopause which is found near $100 \mathrm{~km}$ altitude.

The water vapor molecule contributes to refractivity both through its polarizability and its large permanent dipole moment. The water vapor contribution to refractivity in (44) is

$$
\mathrm{c}_{2} n_{w}=\left(\mathrm{c}_{\mathrm{w} 1}+\mathrm{c}_{w 2} / T\right) n_{w},
$$

where $T$ is temperature in Kelvin. The inverse temperature dependence reflects the random orientation of the permanent dipole moment associated with the molecule's rotational energy (Atkins, 1983, p. 356).

Since density is proportional to partial pressure divided by temperature (via the ideal gas law), the dry molecule and water vapor contributions to refractivity can be written as (Thayer, 1974)

$$
N=77.60\left(P_{a} / T\right) Z_{a}^{-1}+64.8\left(P_{w} / T\right) Z_{w}^{-1}+3.776 \times 10^{5}\left(P_{w} / T^{2}\right) Z_{w}^{-1},
$$

where $P_{a}$ and $P_{w}$ are the partial pressures of the dry and wet molecules respectively in mbar. $Z_{w}$ and $Z_{a}$ are compressibility factors given by Owens (1967) representing the non-ideal properties of the gas. Smith and Weintraub (1953) expressed (45) as a two-term approximation:

$$
N=77.6 \frac{P}{T}+3.73 \times 10^{5} \frac{P_{w}}{T^{2}},
$$

which uses the more readily measurable quantity, the total pressure, $P$, which is $P_{a}+P_{w}$. In (46), the first term accounts for the polarizability of the dry molecules and slightly overestimates the polarizability of water vapor molecules. Therefore, the constant in the permanent dipole term term in (46) has been slightly reduced relative to that in (45). As discussed in Kursinski et al. (1997), the accuracy of (46) is sufficient for most purposes.

The relatively high dielectric constant of particles of condensed water suspended in the atmosphere slows the light propagation via scattering. Because the particles are much smaller than the GPS wavelengths and therefore safely in the Rayleigh scattering regime, the refractivity is proportional to the mass of condensed water in the atmosphere, $W$, and independent of particle size distribution. A first order discussion is given by Kursinski (1997). For liquid water droplets, the refractivity is $1.4 \mathrm{~W}$ where $W$ is in grams per cubic meter. For ice crystals the scale factor is roughly 0.6 rather than 1.4 (Kursinski, 1997). A more detailed form of the refractivity expression for liquid water is given by Liebe (1989).

Refractivity in the ionosphere is due to free electrons and is dispersive. To first order the ionospheric refractivity is given as $40.3 \times 10^{6} n / f^{2}$ where $n_{e}$ is the electron number density per cubic meter, $f$ is transmitter frequency in Hertz. The full Appleton-Hartree expression for ionospheric refractivity is given in Papas (1965). A more complete power series expansion can be found for example in Bassiri and Hajj (1993). The ionosphere contribution to refractvity becomes important at altitudes above 60 to $90 \mathrm{~km}$.

The combined four contributions to refractivity are given to first order by

$$
N=(n-1) \times 10^{6}=77.6 \frac{P}{T}+3.73 \times 10^{5} \frac{P_{w}}{T^{2}}+4.03 \times 10^{7} \frac{n_{e}}{f^{2}}+1.4 \mathrm{~W} .
$$


Throughout this paper, the four refractivity terms in (47) are referred to as the dry, moist, ionospheric, and scattering terms. For realistic suspensions of liquid water or ice, the scattering term is small in comparison with the other terms and is therefore neglected in general (Kursinski, 1997). The dry term dominates the refractivity below $\sim 60-90 \mathrm{~km}$. As a result of its large permanent dipole moment, water vapor produces 15 to 20 times the refractivity per molecule as a dry molecule and it contribution to refractivity becomes significant in the lower roposphere. Furthermore, since the scale height of water vapor can be much smaller than that of the dry atmosphere, water vapor can dominate the vertical gradient of refractivity and therefore the observed bending angles and signal levels in the lower troposphere.

A representative example of atmospheric bending as a function of ray path tangent height is shown in Fig. 11. The first curve is bending due to the vertical refractivity structure of the U.S. Standard Atmosphere. Bending decreases rapidly with tangent altitude, from $1^{\circ}$ to $2^{\circ}$ near the surface to $-2 \times 10^{-5} \mathrm{deg}$. at $80 \mathrm{~km}$ where the bending is roughly equal to the measurement precision. The small size of measurement error relative to bending angle magnitudes in the lower atmosphere demonstrates the extreme precision with which atmospheric delay can be determined.

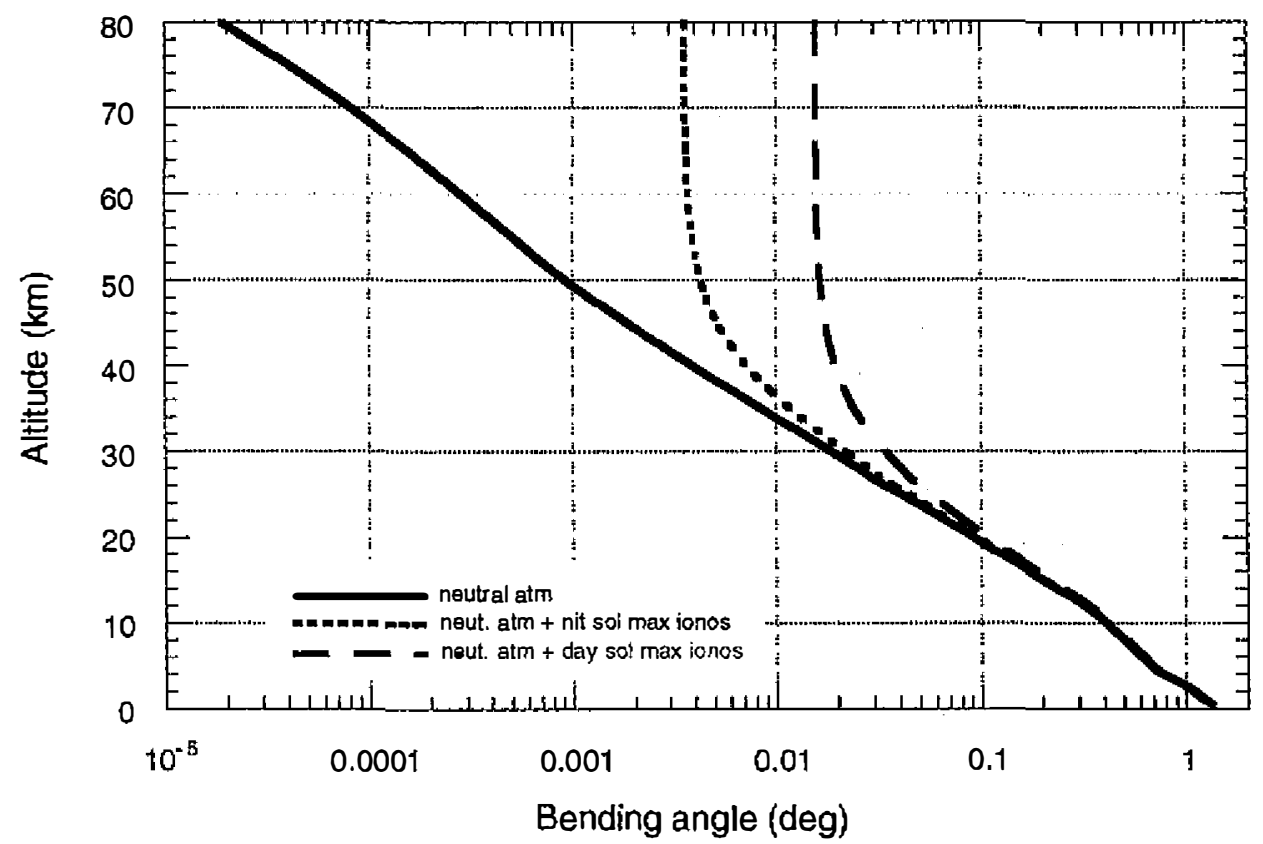

Fig. 11. Atmospheric and ionospheric bending for limb ray paths plotted as a function of ray path tangent height. Solid line: bending calculated for U.S. Standard Atmosphere. Long dashed line: bending calculated for U.S. standard atmosphere plus typical mid-latitude ionosphere during daytime, solar maximum conditions. Short dashed line: bending calculated for U.S. standard atmosphere plus typical mid-latitude ionosphere during nighttime, solar maximum conditions. 
The second and third curves in Fig. 11 include the contribution of the ionosphere based on Chapman layer representations of the $E$ - and $F$-region electron density profiles. The second and third curves include the ionospheric bending under nighttime and daytime conditions, respectively, at the maximum of the solar cycle. Ionospheric bending depends weakly on altitude for tangent altitudes below $80 \mathrm{~km}$ because ionospheric refractivity gradients are greatest above $90 \mathrm{~km}$, significantly above the ray path tangent point. Under daytime conditions during the maximum of the solar cycle, ionospheric bending exceeds atmospheric bending above $30 \mathrm{~km}$ and therefore must be well calibrated to derive accurate profiles in the middle atmosphere.

It is clear from (47) that there is ambiguity between the contributions of the dry, moist, and ionospheric terms to refractivity. Separation and removal of the dispersive ionospheric contribution in (47) using the dual GPS transmitter frequencies L1 and L2 is described in detail in Section 9.

\subsection{Derivation of Pressure and Temperature}

After correcting for the ionosphere, only the dry and moist atmospheric contributions to refractivity remain. In regions where the water vapor mixing ratio is less than $\sim 10^{-4}$, the moist term can be neglected and the refractivity is proportional to the dry number density and (47) reduces to $N=77.6 P / T$. Combining the dry refractivity with the equation of state (the ideal gas law) yields the relationship between density and refractivity:

$$
\rho(r)=\frac{N(r) m}{b_{1} R}=\frac{P(r) m}{T(r) R},
$$

where $R$ is the gas constant, $m$ is the mean molecular mass of dry air, $\rho(r)$ is the air density in $\mathrm{kg} \mathrm{m}^{-3}$, and $b_{1}=77.6 \mathrm{~N}$-unit $\mathrm{K} \mathrm{mbar}^{-1}$. Air below the homopause is well mixed and $m$ is a known constant for dry air so that pressure can be obtained from density by integrating the equation of hydrostatic equilibrium:

$$
\partial P / \partial r=-g \rho,
$$

where $g$ is the acceleration due to gravity. (49) means the pressure can be determined to an unknown constant via hydrostatic balance given the density derived from the refractivity derived from the Abel integral. The simplest solution to determining the unknown constant of integration is to take the temperature from an analysis or climatology at the height at which the hydrostatic integral is to begin. This temperature combined with the density derived from the Abel transform and (48) yields the pressure via the ideal gas law at the top of the hydrostatic integral. Fractional pressure errors due to the temperature guess decrease rapidly as the integration moves deeper into the atmosphere (Kursinski et al., 1997), and accurate temperature profiles can be derived from profiles of $\rho$ and $P$ throughout the stratosphere down to the midtroposphere and below depending on latitude.

The presence of significant tropospheric water vapor complicates the interpretation of refractivity. The dry contribution to refractivity can be isolated given estimates of humidity yielding accurate profiles of density, pressure, and temperature. This works well in the colder 
tropospheric regions, where water vapor concentrations are small. From (47) and the gas law,

$$
\rho=\frac{m}{b_{1} R}\left(N-\frac{b_{2} P_{w}}{T^{2}}\right),
$$

where $b_{2}=3.73 \times 10^{5} \mathrm{~K}^{2} \mathrm{mbar}^{-1}$, and $m$ is now the mean molecular mass of moist air. Pressure and temperature profiles are derived from the density profile using (49), and the new temperature and mean molecular mass profiles are then used in (50) to update the estimate of $\rho$. This process is repeated iteratively a few times until the derived temperature profile converges. Water vapor concentration estimates can be obtained from independent observations, weather analyses or climatology. Sensitivities to water vapor uncertainties are discussed in Kursinski et al. (1997) briefly in Section 10.

\subsection{Derivation of Water Vapor}

Deeper in the troposphere, water vapor concentrations increase contributing up to $30 \%$ to net refractivity and can locally dominate the vertical refractivity gradients and bending near the surface under warm conditions. (46) can be rearranged to solve for water vapor, so that

$$
P_{w}=\frac{N T^{2}-b_{1} P T}{b_{2}} .
$$

The recovery of water vapor from measurements of $N$, using (51), requires independent knowledge of temperature from climatology, independent observations, or weather analyses. The vertical scales of water vapor variability, generally much smaller than that of the dry atmosphere, may also be used in constraining the wet and dry contributions to refractivity. Given $T(r), P_{w}(r)$ and $P(r)$ can derived iteratively from (49), (50), and (51).

\subsection{Optimal Estimation of Water Vapor, Temperature and Pressure by Combining of GPS Profiles with Apriori Analyses}

In regions of the troposphere where wet contributions to refractivity are significant (i.e., q $\geq 0.1 \mathrm{~g} / \mathrm{kg}$ ), the wet and dry contributions to observed refractivity cannot be separated without additional information or constraints. A simple approach for deriving water vapor, temperature and pressure is to combine the occultation profile and the additional information in a least squares framework that uses the information in each in an optimal fashion based upon their error covariances. Global weather analyses like those generated by the European Center for Medium-range Weather Forecasts (ECMWF) or the National Center for Environmental Prediction (NCEP) are likely the best source of information for combining with the GPS profiles because of their global coverage and availability every 6 hours which makes interpolation to the time and location of any occultation easy and straightforward. Furthermore estimates of their error covariances exist.

Following the discussion of Healy and Eyre (2000), the least squares approach begins by defining a penalty function which combines a vector of the measurements from an occultation profile with a state vector consisting of independent estimates of the variables of interest, in 
this case a profile of pressure, temperature and moisture. The least squares solution is the state vector consisting of the values which minimize the penalty function. Assuming all errors are Gaussian, the maximum likelihood penalty function is

$$
\xi(x)=\left(y_{o b s}-y(x)\right)^{T} S_{y}^{-1}\left(y_{o b s}-y(x)\right)+\left(x-x_{a}\right)^{T} S_{a}^{-1}\left(x-x_{a}\right),
$$

where $y_{o b s}$ is the vector of occultation measurements (a profile of either refractivity or bending angles), $y(x)$ is the simulated vector of measurements based on the solution state vector $x, x_{a}$ is the apriori state vector from an analysis, $S_{a}$ is the analysis error covariance and $S_{y}$ is the measurement error covariance plus the covariance of the forward model which relates the state vector to the observations. Notice that by normalizing the measurements and state variables by their respective error covariances, two very different sets of variables have been combined into a single quantity in (52). The solution vector, $x$, represents a profile of temperature and water vapor plus a surface pressure. Pressure at each level is defined by the hydrostatic equation. In the case where the vector, $y$, is a profile of refractivity, the forward model is simply the refractivity equation, (46). If $y$ is a profile of bending angles, the forward model also includes the propagation of the GPS signals through the atmosphere modeled for example by raytracing. The least squares solution for $x$ is that which minimizes the penalty function, $\xi(x)$, such that

$$
\nabla_{x} \xi(x)=S_{a}^{-1}\left(x-x_{a}\right)-K^{T} S_{y}^{-1}\left(y_{o b s}-y(x)\right)=0,
$$

where $K$ is $\nabla_{x} y$, the gradient of $y$ with respect to $x$. Since $y(x)$ is nonlinear we must linearize $y(x)$ and iterate to find the solution which is given as

$$
x_{n+1}=x_{n}-\left[H_{x} \xi(x)\right]^{-1} \nabla_{x} \xi\left(x_{n}\right),
$$

where $n$ is the iteration index and $H$ is the Hessian operator which is obtained by differentiating $\nabla_{x} \xi\left(x_{n}\right)$ again and retaining only first derivative terms:

$$
H_{x} \xi(x) \cong S_{a}^{-1}+K^{T} S_{y}^{-1} K \text {. }
$$

The error covariance of the solution, $x$, is

$$
\hat{S}=\left[S_{a}^{-1}+K^{T} S_{y}^{-1} K\right]^{-1} .
$$

Figures $12 \mathrm{a}, 12 \mathrm{~b}$ and $12 \mathrm{c}$ provide some visual aid in understanding the accuracy of a least squares solution which combines occultation refractivity with analyzed temperatures and water vapor. Each figure is at a specified pressure level in the atmosphere and contains two error ellipses. The larger ellipse is representative of errors in the analyses using $1.5 \mathrm{~K}$ and $25 \%$ as the errors in analysis temperature and water vapor estimates. The smaller or narrower ellipse in each figure indicates the error ellipse of the least squares solution which combines the analysis temperatures and water vapor with the occultation refractivity information. The long axis of the narrow ellipse is oriented along the line of constant refractivity equal to the occultation refractivity. The length of the long axis is approximately equal to the width of the original error ellipse since the refractivity places no constraint along that direction. The short axis is orthogonal to the line of constant refractivity and its length reflects the \pm 1 sigma uncertainty in the measured refractivity that is clearly substantially smaller than the uncer- 
tainty in analyzed variables.

The three figures show cases representative of the upper troposphere/lower stratosphere $(100 \mathrm{mb})$, middle to upper roposphere $(300 \mathrm{mb})$ and lower troposphere $(800 \mathrm{mb})$ demonstrating how refractivity primarily constrains temperature in the cold and dry upper troposphere (Fig. 12a) whereas it primarily conswains water vapor in the lower troposphere (Fig. 12c) and constrains a combination of the two over the intervening altitudes (Fig. 12b).

\subsection{Using GPS Profiles of Bending Angles or Refractivity}

GPS profiles can be in terms of bending angles or refractivity each with its own set of advantages and disadvantages. Refractivity profiles have the advantage of being derived very quickly via the Abel transform. The disadvantage of a 1-D refractivity profile is that accuracy is reduced when significant non-spherical structure is present in the vicinity of the occultation such as in moist regions of lower troposphere. In the moist lower troposphere, a raytracing

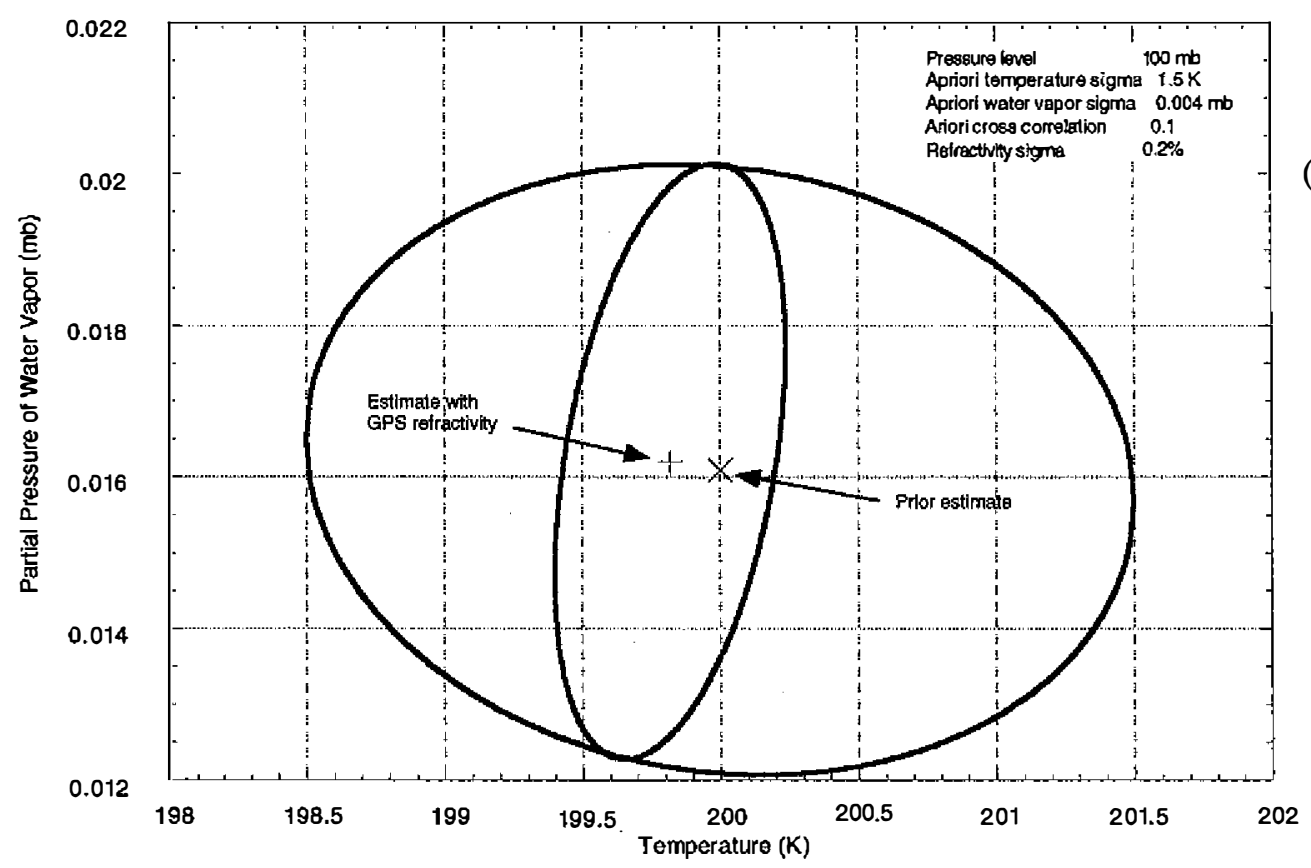

Fig. 12. Error ellipses before and after combining apriori analyses with occultation refractivity data. The larger ellipse represents the $1 \sigma$ error in apriori analysis. The smaller ellipse is the $1 \sigma$ error after combining the apriori analysis with the occultation-derived refractivity profile. (a). $100 \mathrm{mb}$ level with the apriori $\sigma_{\mathrm{T}}=1.5 \mathrm{~K}$, a $25 \%$ water vapor error of $0.004 \mathrm{mb}$ and $\sigma_{\mathrm{N}} \mathrm{N}=0.2 \%$. (b). $300 \mathrm{mb}$ level with the apriori $\sigma_{\mathrm{T}}=1.5 \mathrm{~K}$, a $25 \%$ water vapor error of $0.125 \mathrm{mb}$ and $\sigma_{N} / \mathrm{N}=0.2 \%$. (c). $800 \mathrm{mb}$ level with the apriori $\sigma_{\mathrm{T}}=1.5 \mathrm{~K}$, a $25 \%$ water vapor error of $2.25 \mathrm{mb}$ and $\sigma_{\mathrm{N}} \mathrm{N}=$ $1 \%$. 

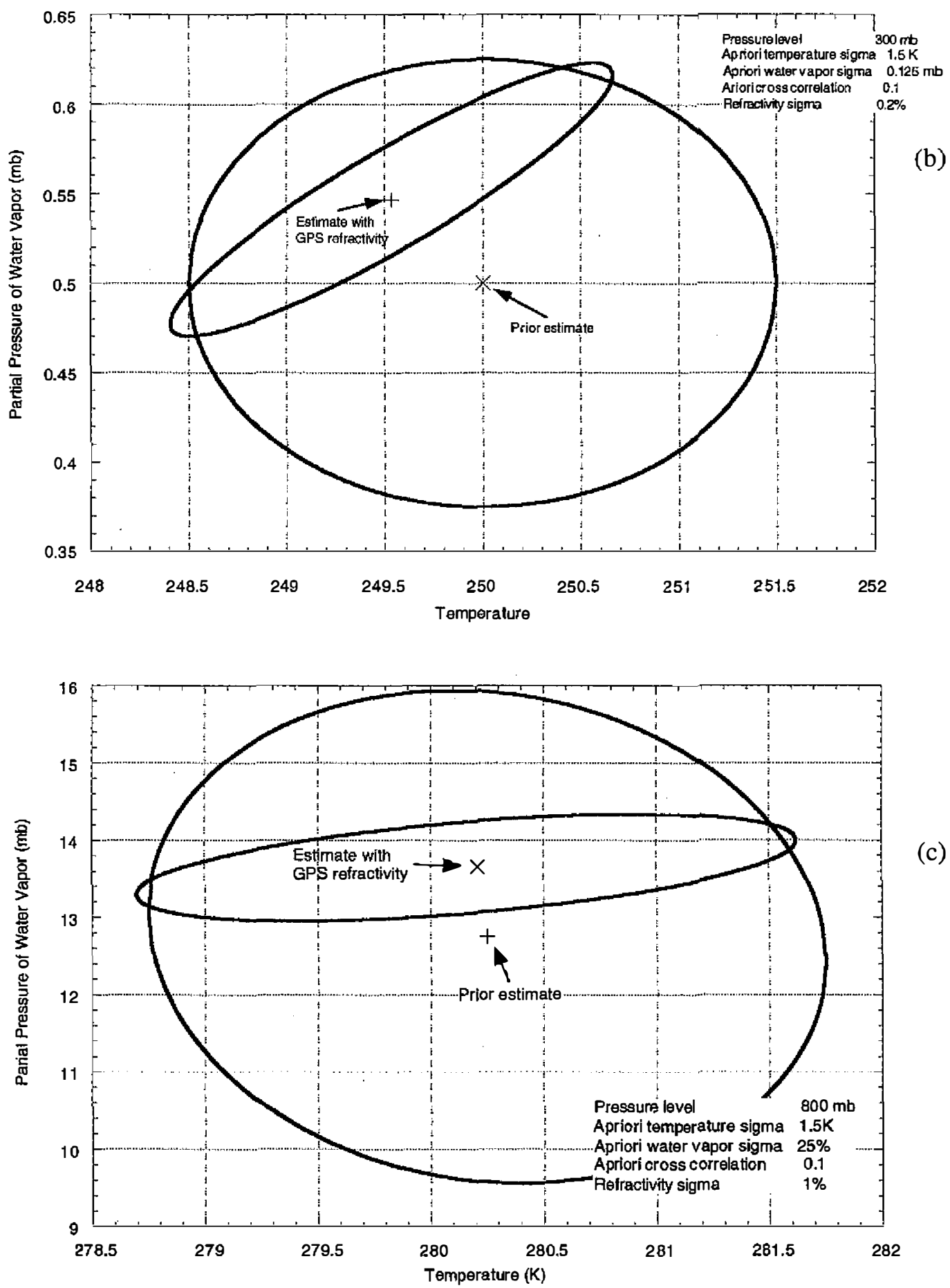

(c)

(Fig. 12. continued) 
scheme that accounts for the 3-D refractivity structure will produce the more accurate results. The disadvantage of a bending angle approach is the computing cost of the forward model particularly given the iterations to deal with nonlinearities. Above roughly $5 \mathrm{~km}$ altitude and most of the moisture in the middle troposphere, the refractivity structure is generally very close to spherical (Kursinski et al., 1995) such that additional complexity of the bending angle approach should yield little benefit over the Abel transform approach.

\subsection{Vertical Sampling and Resolution}

Another point is related to the two sets of heights in (52), those of the occultation profile and those of the apriori analysis. As defined in (52), the vertical levels of the solution state vector, $x$, are those of $x$, the apriori state vector estimate derived in the analysis. Analyses are generally at fixed pressure (or sigma) levels. In contrast, occultation profile heights vary from occultation to occultation as necessary to represent the vertical resolution of each profile which depends on the refractivity structure in the vicinity of each profile (see Section 9). At present, the occultation observations generally have better vertical resolution than the global analyses. Therefore a solution derived only at the analysis levels will have lost some of the occultation information.

Retaining the vertical resolution of the occultations in the solution state vector, $x$, requires that the analysis profile, $x_{a}$, be interpolated to the heights of the occultation profile when used in (52). Its error covariance, $S_{a}$, therefore must also reflect the increase in the number of vertical levels. Simply adding levels to the error covariance and filling the new levels with interpolated values may create zero eigenvectors such that the interpolated $S_{a}^{-1}$ is not well defined. One can circumvent the problem with more indirect and clever methods of approximating the matrix inverse. However, to make full use of the vertical information in the occultation profiles, the error covariance of the prior information should be adjusted to reflect sharp vertical structure apparent in the occultation data. In doing so, the minimization process will be given sufficient freedom to use the occultation's vertical information in the solution, $x$. Modifying $S_{a}$ in such a manner is an area of research. Once a higher resolution solution is achieved, one can smooth $x$ down to the resolution of the analysis levels (and lose some occultation information in the process) if the goal is limited to improving analyses at their predefined levels such as in data assimilation for weather prediction.

Interpolating $x_{a}$ to the levels of the occultation may prove very important when bending angles are assimilated because the vertical resolution of the apriori analysis will be inadequate to represent sharp vertical features of ten apparent in the occultations. In such conditions, if the vertical resolution of the apriori analysis and solution vectors is not increased, the least squares solution of (52) may be quite poor. The reason is that minimization of the penalty function will increase the solution's vertical refractivity gradients in order to create the large bending angles of the observations. To do so given the limited number of vertical intervals of the analysis, requires that the vertical gradients extend over larger vertical intervals than exist in reality. The result will be an ugly compromise between the apriori solution and the observations yielding a high penalty function value, $\xi(x)$, in (52) and a solution vector, $x$, which may be farther from reality than the apriori state vector. The problem is simply that the analyzed 
levels do not provide the degrees of freedom necessary to represent basic properties of the observations. One approach to reduce the erroneous behavior is to penalize sharp vertical structure in the occultation data by increasing its error covariance. Such an approach, while better behaved, is clearly suboptimal in that it intentionally throws away potentially important occultation information.

\section{SOME DETAILS OF PROCESSING REAL OCCULTATION DATA}

Here we describe the occultation phase observable and how the portion of the phase delay due to the atmosphere is isolated from other terms. We also describe the initialization of the Abel transform and the hydrostatic integrals. Our discussion largely summarizes that of Hajj et al. (2000).

\subsection{Removal of Reference Clock Instabilities}

The primary observables used during an occultation experiment are the $\mathrm{C} / \mathrm{A}$ and $\mathrm{P} 2$ phase measurements between the low-Earth orbit (LEO) satellite and the occulting GPS satellite (see Section 2). To an arbitrary constant, these phase measurements can be thought of as measurements of the range between the transmitter and receiver that contain the contribution of the propagation delay caused by the atmosphere. Since we are ultimately interested in deriving the Doppler shift or range rate from these observations to determine the bending angle (Section 3.5), the unknown constant is not important. Our challenge at this point is to isolate the portion of the observed delay due to the light travel time between the transmitter and the receiver.

The first problem is the observed phase propagation delay between the transmitter and receiver (including the effect of the atmosphere) is degraded by instabilities in the reference clocks. While the error due to the instability of the GPS satellite atomic frequency standards is small (Kursinski et al., 1997), the instability of inexpensive crystal frequency references used on the LEO GPS receivers and the Selective Availability (SA) encryption on the transmitter signal are important sources of error. These errors can be eliminated by differencing or, more correctly, by estimating and removing the erroneous behavior. The required general receiver and transmitter geometry is shown in Fig. 13.

Reduction of instabilities in the LEO receiver clock is accomplished by tracking a second GPS satellite (GPS (3) in Fig. 13) simultaneously during each occultation. Differencing the measured GPS (2) and GPS (3) phases eliminates the instability of the receiver clock while adding the instabilities of the GPS (3) clock, which can be 3 orders of magnitude more stable than the receiver clock. Thus, at the cost of doubling the data volume and increasing the complexity of the processing, the need for a high quality receiver clock is eliminated, which significantly reduces the size, power and cost of LEO receiver instrumentation. Furthermore, the data doubling penalty can be eliminated by performing the first step of the clock correction process in the LEO receiver, that is, the differencing of the GPS (2) and GPS (3) phases, within the LEO receiver before telemetering the data to the ground.

While the GPS transmitter atomic clocks are inherently quite stable, the Selective Availability (SA) encryption which is intentionally added to the GPS signals creates apparent wan- 


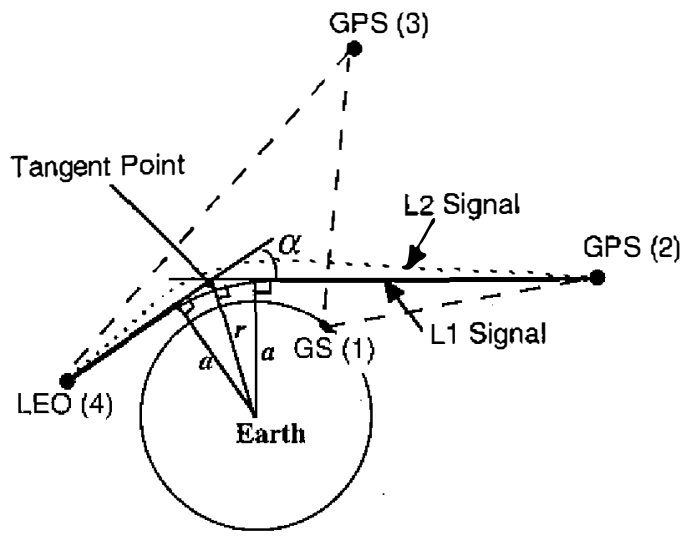

Fig. 13. GPS occultation geometry defining the clock calibration geometry including the non-occulting GPS transmitter and ground receiver used for calibration. Also depicted is the slight separation between the L1 and L 2 signal paths due to the dispersive ionospheric refractivity.

der in the GPS satellite positions and clocks of the order of 100 meters or more over hundreds of seconds. While the SA modulation can be eliminated with a classified facility, an unclassified approach is to estimate and remove the SA modulation and inherent clock instabilities using a second receiver which tracks the GPS (2) and GPS (3) satellites during each occultation (Hajj and Kursinski, 1991, Hardy et al. 1994). By sampling once per second and cubically interpolating the results, phase errors due to the SA modulation can be reduced to less than 0.1 $\mathrm{mm}$ (Wu et al., 1990). The second receiver for correcting SA (GS (1) in Fig. 13) is shown as being on the ground in Fig. 13. However, the anticipated number of receivers in LEO during the COSMIC mission time frame may be sufficient to allow the second receiver to be an orbiting receiver which would eliminate the occultation requirement for the 1 second ground receiver network. If the SA encryption were turned off, as is being discussed, the stability of the GPS transmitters would be sufficient to eliminate the requirement for the second receiver (Kursinski et al., 1997).

Another penalty of the clock correction scheme in addition to the increased complexity and data volume is the additional noise associated with each additional link. The noise penalty as discussed by Kursinski et al. (1997) scales approximately as the square root of the number of links involved assuming the phase quality of the additional links is comparable to that of the occultation link. In comparison to the SA noise and noise of the relatively unstable, inexpensive crystal oscillators, the additional noise penalty is minor.

We now summarize the clock estimation and removal process described in detail by Hajj et al. (2000) that includes the correction for relativistic effects and differential light times between the four links shown in Fig. 13. These steps are performed using the GIPSY/OASIS software developed at JPL for precise positioning and orbit determination applications using GPS. Hajj et al. distinguish between three types of time: (a) clock time, the time recorded by the transmitter's or receiver's clock (referred to as a time tag) which contains a time varying offset; (b) proper time, the time recorded by a perfect clock in a frame moving with the transmitter or receiver; and (c) coordinate time, the time recorded by a perfect clock in a given coordinate system.

A GPS receiver measures the range between the transmitter and the receiver by essentially differencing the transmitter's time tag associated with a given sequence of code from the receiver's time tag at the time that sequence is received. Up to a constant bias, phase measure- 
ments can be thought of in the same manner. The measured difference between the received and transmit time can be modeled as the sum of five terms: 1) the light travel time, 2) the receiver's clock error, 3) the transmitter's clock error, 4) the proper time minus coordinate time at the receiver (due to special and general relativistic effects), and 5) the coordinate time minus proper time at the transmitter.

In an occultation geometry, the term of interest is the light travel time associated with the $L^{24}$ link in Fig. 13 which includes the extra delay caused by the atmosphere. To isolate the occultation light travel time, we must either solve for or compute all the other terms. To begin with, we essentially form the difference between the measured $L^{34}$ and $L^{24}$ delays canceling the clock 4 errors that are common to both links. Because the LEO receiver clock (4) is by far the worst of the four clocks involved, it is important that the LEO receiver (4) measure the $L^{34}$ and $L^{24}$ phase delays over identical time intervals such that the clock (4) errors cancel exactly. The $L^{34}$ minus $L^{24}$ delay difference we formed contains unwanted contributions from errors in the GPS (2) and (3) clocks, the coordinate versus proper time differences for GPS (2) and (3) and the light travel time for $L^{34}$.

We can estimate the light time for $L^{34}$ and the other two non-occulting links in Fig. 13 as well as the differences between coordinate and proper times for all links using the precise orbit solutions of both transmitters and receivers. The orbits are derived using a ground network of fiducials and all other GPS satellites. Given these estimates, we can subtract the contribution of the GPS (3) to LEO (4) light travel time and receiver and transmitter coordinate and proper time differences from the measured $L^{34}$ minus $L^{24}$ delay difference which leaves a residual consisting of the desired light travel time between GPS (2) and LEO (4) plus the unwanted contributions of the GPS (2) and (3) clock drifts.

To solve for the GPS (2) clock drift, we subtract the estimated GPS (2) to GS (1) light travel time and GPS (2) and GS (1) coordinate minus proper times from the measured delay $L^{21}$ which yields the drift of clock (2) relative to clock (1). We solve for the drift of clock (3) relative to clock (1) similarly using the measured delay, $L^{31}$. The epochs at which clocks (2) and (3) are solved for differ slightly from the transmission times of the $L^{34}$ and $L^{24}$ links because of differences between the link distances. Therefore we first interpolate the clock (2) and (3) drifts to the desired transmission times and then subtract them from the residual yielding our best estimate of the desired GPS (2) to LEO (4) light travel time. Note that since the clock (2) and (3) solutions are both relative to clock (1) the final solution is essentially independent of clock (1) as long as the clock (1), (2) and (3) errors are sufficiently smooth between samples to enable accurate interpolation.

\subsection{Deriving Atmospheric Doppler}

The calibration process extracts the sum of the neutral atmospheric and ionospheric delays for both the L1 and L2 occulted links. The atmospheric delay is normally obtained at a high rate $(50 \mathrm{~Hz}$ for the GPS/MET experiment) chosen to meet or exceed the Nyquist rate needed to extract multiple simultaneous signals in the lower troposphere (see Section 6), and for sampling the diffraction pattern induced by the atmosphere for high resolution retrievals (see Section 7). Here we discuss the approach to derive the atmospheric Doppler shift in order 
to derive a profile of bending angles for the Abel inversion technique consistent with the resolution inherent in the geometric optics approximation. In order to maximize the vertical resolution of the derived bending angle profile while not exceeding the resolution limit of geometric optics discussed in Section 4.3, we estimate the Doppler frequency smoothed over an interval corresponding to the time for the tangent height of a ray path to descend or ascend the diameter of the first Fresnel zone $(=F)$. The Fresnel diameter of the occulted signal can be determined based on L1 and L2 amplitude measurements (Haugstad; 1978; Hajj et al., 2000). The Fresnel zone diameter is $F=F_{0} M^{1 / 2}$ as defined in (22) and (21). M, which is defined in (18), can be estimated from the observed signal intensity as given by (19). Given knowledge of the rate of descent of the raypath tangent point in the absence of the atmosphere $\left(V_{0}\right)$ and knowledge of $M$, we can estimate the rate of descent of the raypath tangent point, $V$, during the occultation

$$
V=V_{0} M,
$$

Combining (54) and (22) into (55) yields the time for the raypath tangent height to descend a Fresnel zone, $T_{F}$.

$$
T_{F}=\frac{F}{V}=\frac{F_{0}}{V_{0} \sqrt{M}} .
$$

Using a smoothed version of the received signal intensity, $I$, measured during an occultation and the signal intensity at the beginning of the occultation to represent $I_{0}$, we can compute $M$ from (18) and (19). A weather analysis or climatological model can be used to estimate and remove the pressure and temperature absorption term in (19) in the lower troposphere. The absorption term can be further refined with an iteration through the retrieval process if desired. $T_{F}$ is chosen as the interval for estimating the L1 and L2 atmospheric Doppler shifts from highrate $\mathrm{L} 1$ and $\mathrm{L} 2$ phase to avoid the diffraction effects from sub-Fresnel atmospheric structure appearing in the retrieval. The time step between Doppler estimates is chosen to be 2 to 3 times smaller than $T_{F}$ to reduce errors in interpolation in subsequent numerical integrals like the Abel transform. The Doppler shift is estimated independently for each GPS frequency.

Given thermal noise on the phase measurements which is independent from sample to sample, the error in the phase rate (=Doppler) estimated using $m$ phase measurements, is proportional to $\mathrm{m}^{-3 / 2}$ such that

$$
\sigma_{f} \alpha \tau^{-3 / 2},
$$

where $\sigma_{f}$ is the standard deviation of the Doppler frequency error and $\tau$ is the integration time over which the frequency is estimated. The inverse $3 / 2$ power scaling is essentially because increasing the number of points by a factor of $m$ reduces the error in the average phase estimate in proportion to $m^{-1 / 2}$ and taking the derivative over the time interval equal to $m$ sample intervals introduces an additional factor of $m^{-1}$. The $-3 / 2$ power scaling of the occultation Doppler error creates a strong tradeoff between resolution and accuracy when the thermal noise dominates as can occur at high altitudes. For example increasing the integration time by a factor of 2 reduces the resolution by a factor of 2 and reduces the Doppler error by a factor of 2.8. This means the altitude at which a specific Doppler or equivalently bending angle error is achieved with the original smoothing interval is raised by roughly a scale height when the 
smoothing interval is doubled. Such a tradeoff can be particularly important when characterizing the middle atmosphere.

\subsection{Ionospheric Calibration}

Although the neutral atmosphere is non-dispersive at radio frequencies, the electromagnetic signals travel through the dispersive ionosphere on their way into and out of the lower neutral atmosphere, causing the $\mathrm{L} 1$ and $\mathrm{L} 2$ signals received at a given time to sense slightly different parts of the neutral atmosphere (as depicted by the solid, L1, and dotted, L2, occulted signals of Fig. 13). The separation of the two signals at ionospheric heights near the tangent point varies between less than a hundred meters to $5 \mathrm{~km}$, depending on the tangent height of the occulted signal, the solar conditions, local time and the location of the occultation. (For this effect and other ionospheric effects on GPS occultations see, e.g., Hajj and Romans, 1998.) In the most general situation, an ionospheric correction is needed in order to estimate the neutral atmospheric bending. We follow a procedure first suggested by Vorob'ev and Krasil'nikova (1994). Let $\alpha_{1}\left(\boldsymbol{a}_{1}\right)$ and $\alpha_{2}\left(a_{2}\right)$ be the bending as a function of the asymptotic miss distance for the $\mathrm{L} 1$ and $\mathrm{L} 2$ signals, respectively. The linear combination

$$
\alpha_{\text {neut }}\left(a_{0}\right)=\frac{f_{1}^{2}}{\left(f_{1}^{2}-f_{2}^{2}\right)} \alpha_{1}\left(a_{0}\right)-\frac{f_{2}^{2}}{\left(f_{1}^{2}-f_{2}^{2}\right)} \alpha_{2}\left(a_{0}\right),
$$

where $\alpha_{1}$ and $\alpha_{2}$ have been interpolated to the same value of $a_{\circ}$, removes the first order ionospheric bending (which is proportional to $f^{2}$ ). The purpose of using the same value of $a$ in (57) is to collocate the heights of the L1 and L2 signal path tangent points before forming the ionosphere correction. The two coefficients on the right side of (57) have the numerical values to 2.5457 .. and 1.5457. ., respectively.

Based on (57) and assuming the $\mathrm{Ll}$ and $\mathrm{L} 2$ errors are independent, the noise associated with the neutral atmospheric bending is given by

$$
\sigma_{\alpha_{\text {neut }}}^{2}=(2.54)^{2} \sigma_{\alpha_{\mathrm{s}}}^{2}+(1.54)^{2} \sigma_{\alpha_{2}}^{2} .
$$

$\mathrm{L} 2$ phase measurements are noisier than $\mathrm{L} 1$ phase measurements for several reasons. First, the L2, which has a lower frequency than L1, is more influenced by ionospheric scintillation and delay. Second, the $\mathrm{C} / \mathrm{A}$ code is transmitted with $3 \mathrm{~dB}$ more power that the $\mathrm{P} 1$ code, which in turn is 1 to $3 \mathrm{~dB}$ stronger than the $\mathrm{P} 2$. The relative strength of $\mathrm{C} / \mathrm{A}$ relative to $\mathrm{P} 1$ and $\mathrm{P} 2$ also depends on whether or not the Department of Defense (DoD) anti-spoofing (AS) is activated, and on the type of the receiver and racking strategy. In general, L1 phase derived based on C/ A is more accurate than $\mathbf{L} 2$.

However, even under conditions where both L1 and L2 noise are comparable, (58) implies that the noise introduce by the ionospheric-free linear combination is about a factor of 3 larger than individual signal noise. As pointed out by Hajj et al. (2000), we can reduce this noise amplification by taking advantage of the strong sensitivity of the Doppler (and therefore bending angle) error to the integration time given in (56). To do so, Hajj et al. rewrite the bending in (57) as 


$$
\alpha_{n e u t}\left(a_{0}\right)=\alpha_{1}\left(a_{0}\right)+1.54\left(\bar{\alpha}_{1}\left(a_{0}\right)-\bar{\alpha}_{2}\left(a_{0}\right)\right),
$$

where $\bar{\alpha}_{1}$ and $\bar{\alpha}_{2}$ are the L1 and L2 bending smoothed over longer intervals than the time to cross the diameter of the first Fresnel zone discussed previously. Increasing the smoothing interval by a factor of 3 relative to the Fresnel zone smoothing reduces the amplification to about 1.1 instead of 3 . The penalty of smoothing the ionosphere correction is that the correction no longer removes as much of the fine scale ionosphere effects as the unsmoothed correction. An optimal smoothing interval can be chosen which minimizes the combined contributions of the thermal noise and residual fine-scale, ionosphere effects. The choice can be made on the basis of the spectrum of the high rate L1 - L2 phase variations.

The ionosphere calibration should not be continued above a certain height, when the neutral atmosphere signature on the occulted signal is comparable to residual ionospheric effects or the receiver's thermal noise. Hajj et al. (2000) determine this height by computing a moving $\chi^{2}$ based on departures from an exponential fit to ionospheric-free bending. They find this height tends to occur at a height of order $\sim 50-60 \mathrm{~km}$, depending on the ionospheric conditions. Deep in the atmosphere the L2 signal phase can be very noisy due to defocusing effects. Therefore Hajj et al. suggest that when the $\mathrm{L} 2 S N R_{\mathrm{v}}$ drops below a certain limit, a low order extrapolation of the ionospheric correction from higher altitudes should be used to correct for the ionosphere in the lowermost troposphere.

\subsection{Upper Boundary Conditin of the Abel Transform}

The upper limit of the Abel integral (13) requires that the bending as a function of $a$ extends to infinity. However, as just discussed, the estimated bending is accurate only up to a finite upper height, $\boldsymbol{a}_{u}$. Therefore, Hajj et al. suggest combining the occultation information with information from another source using a simple least-square estimator of $\alpha$ based on measured bending, $\alpha_{\mathrm{m}}$, and estimated bending (from an a priori model), $\alpha_{\mathrm{e}}$, weighted by their corresponding uncertainties, $\sigma_{\mathrm{m}}$ and $\sigma_{\mathrm{e}}$. In order to make the occultation retrieval completely independent of the model below a certain height, $a_{u}, \sigma_{e}$ can be effectively set to infinity below this height.

\section{PREDICTED ACCURACY}

Kursinski et al. (1995, 1997) identified and quantified a number of important sources of error in the occultation measurement and retrieval process which are briefly summarized below.

\subsection{Refractivity Errors}

Figure 14 shows the estimated root-mean-square (RMS) refractivity errors for individual profiles as estimated by (Kursinski et al., 1997). Differences at middle atmospheric altitudes between Figs. 14a and 14b are due primarily to higher signal to noise ratio at the GPS receiver and night time ionosphere conditions assumed in Fig. 14a. The dominant error below $30 \mathrm{~km}$ is 

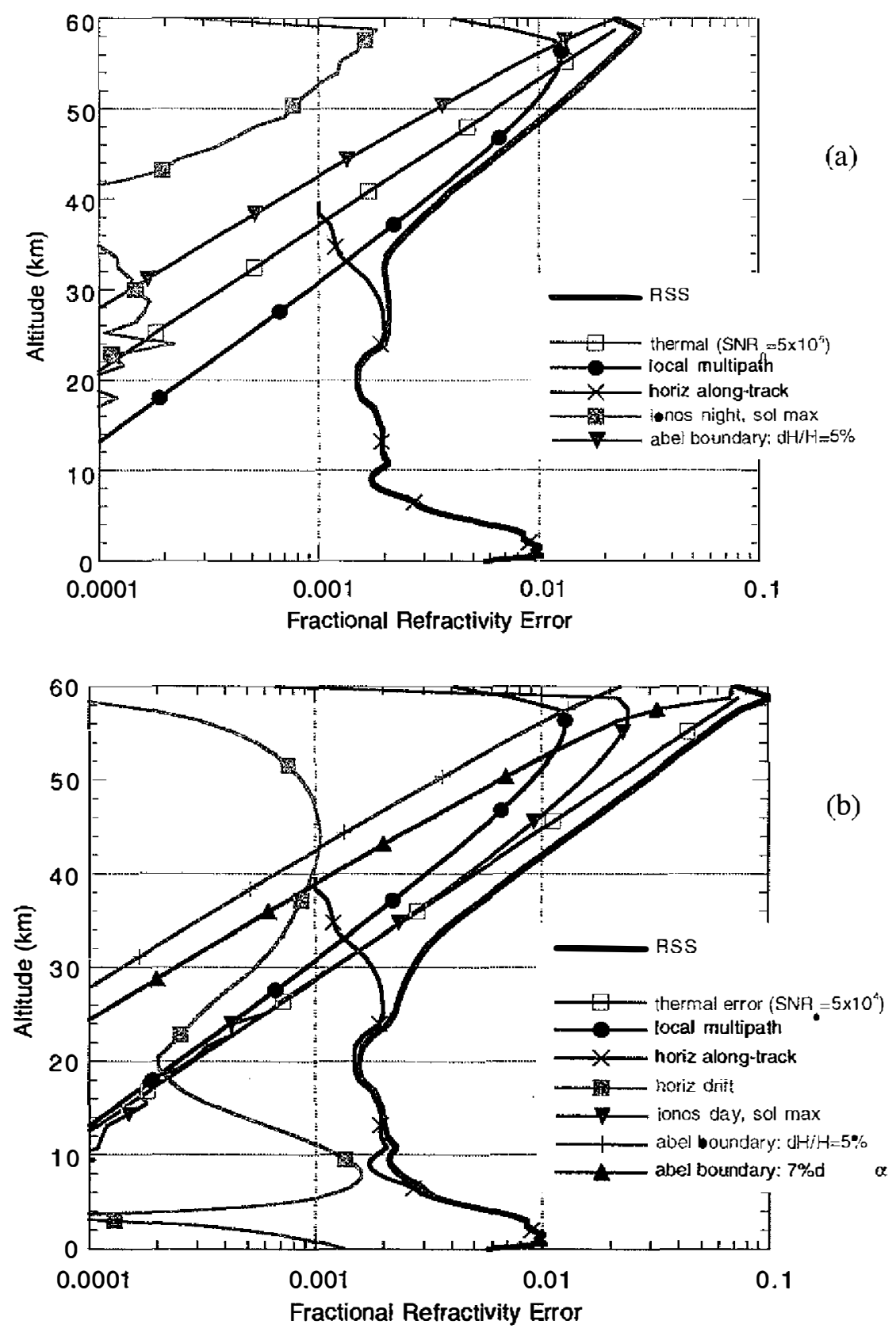

Fig. 14. Summary of predicted refractivity errors versus height from Kursinski

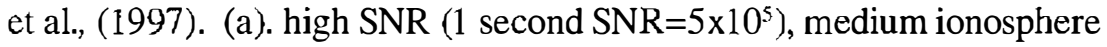
(night time, solar maximum or daytime, solar minimum conditions) and high latitude (small specific humidity). (b). GPS/MET SNR (1 second $\left.\mathrm{SNR}=5 \times 10^{4}\right)$, large ionosphere (daytime, solar maximum ionosphere), and low latitude (high specific humidity). 
non-spherical structure leading to errors of 0.2 to $0.3 \%$ between 6 and $30 \mathrm{~km}$ altitude. Less accurate results in the lower troposphere reflect the influence of horizontal water vapor structure at low altitudes.

\subsection{Temperature Errors}

Figure 15 shows the estimated RMS temperature errors for individual profiles (Kursinski et al., 1997). Differences at middle atmospheric altitudes between Figs. 15a and 15b are due primarily to higher signal to noise ratio at the GPS receiver and night time ionosphere conditions assumed in Fig. 14a. Less accurate results in the lower troposphere in Fig. 15b reflect the uncertainty of water vapor at low latitudes whereas Fig. 15a reflects high latitude conditions. Figures $15 \mathrm{a}$ and $15 \mathrm{~b}$ assume tropospheric water vapor is known to $20 \%$. In the upper troposphere-lower stratosphere, the dominant error is the spherical symmetry assumption. Note that estimated errors in retrieved temperature are sub-Kelvin from the upper troposphere into the lower stratosphere.

\subsection{Geopotential Errors}

Figure 16 shows the estimated RMS error in geopotential heights of pressure surfaces derived from the GPS observations. The primary difference between Figs. 16a and 16b in the middle atmosphere is the nighttime versus daytime ionosphere. In the troposphere the difference represents the impact of water vapor at high versus low latitudes analogous to Figs. 15a and $15 \mathrm{~b}$. Errors are estimated to be 10 to $20 \mathrm{~m} \mathrm{RMS}$ in the lower stratosphere through upper troposphere where the error associated with the spherical symmetry assumption dominates. Small errors extend down to the surface at high latitudes where the air is cold and dry.

\subsection{Water Vapor Errors}

Kursinski et al. (1995) and Kursinski and Hajj (1999) have estimated the accuracy to which tropospheric water vapor profiles can be derived from GPS refractivity profiles. In terms of specific humidity, the RMS error ranges from 0.2 to $0.5 \mathrm{~g} / \mathrm{kg}$ as shown in Fig. 17 which is based in the annual mean conditions from Peixoto and Oort (1992) using the refractivity error versus height in Fig. 14 and assuming RMS temperature and surface pressure errors of $1.5 \mathrm{~K}$ and $3 \mathrm{mb}$ respectively. Accuracy is primarily limited by the temperature accuracy above $4 \mathrm{~km}(\sim 600 \mathrm{mb})$. At lower altitudes, the contribution due to refractivity errors becomes larger because horizontal water vapor variations reduce the accuracy of the spherical symmetry approximation. Fractional accuracy of water vapor is shown in Fig. 18. At low latitudes, humidity derived from individual profiles should be accurate to $20 \%$ up to 6 or $7 \mathrm{~km}$ altitude and to better than $5 \%$ within the convective boundary layer, given independent temperature at accuracies of $\sim 1.5 \mathrm{~K}$ presently available from weather analyses. Fractional accuracies decrease at higher latitudes and during winter conditions such that at $45^{\circ}$ latitude, $20 \%$ accuracy varies from approximately $5 \mathrm{~km}$ in the summer to $2-3 \mathrm{~km}$ in the winter. The accuracy of averaged water vapor for climatological purposes will depend on climatological temperature accuracy and should be 2 to 3 times better than the accuracy of individual water vapor profiles. 

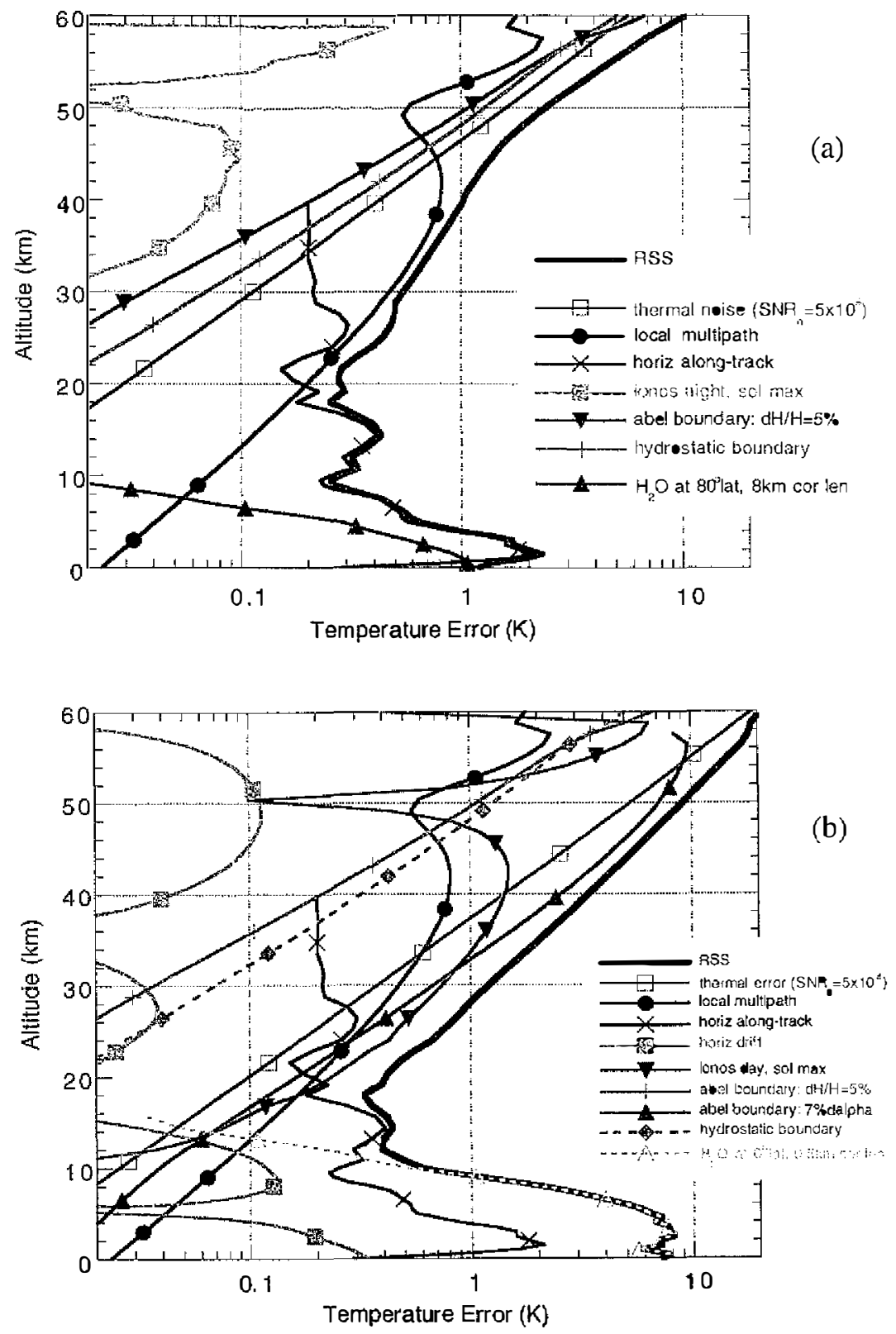

Fig. 15. Summary of predicted temperature errors versus height from Kursinski

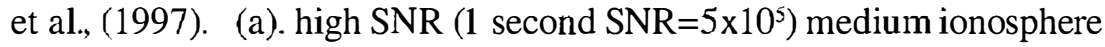
(night time, solar maximum or daytime solar minimum conditions) and high latitude tropospheric water vapor: $80^{\circ}$ latitude with $0.5 \mathrm{~km}$ vertical correlation length. (b). GPS/MET SNR ( 1 second SNR $\left.=5 \times 10^{4}\right)$, large ionosphere (daytime, solar maximum ionosphere), and low latitude (high specific humidity). 

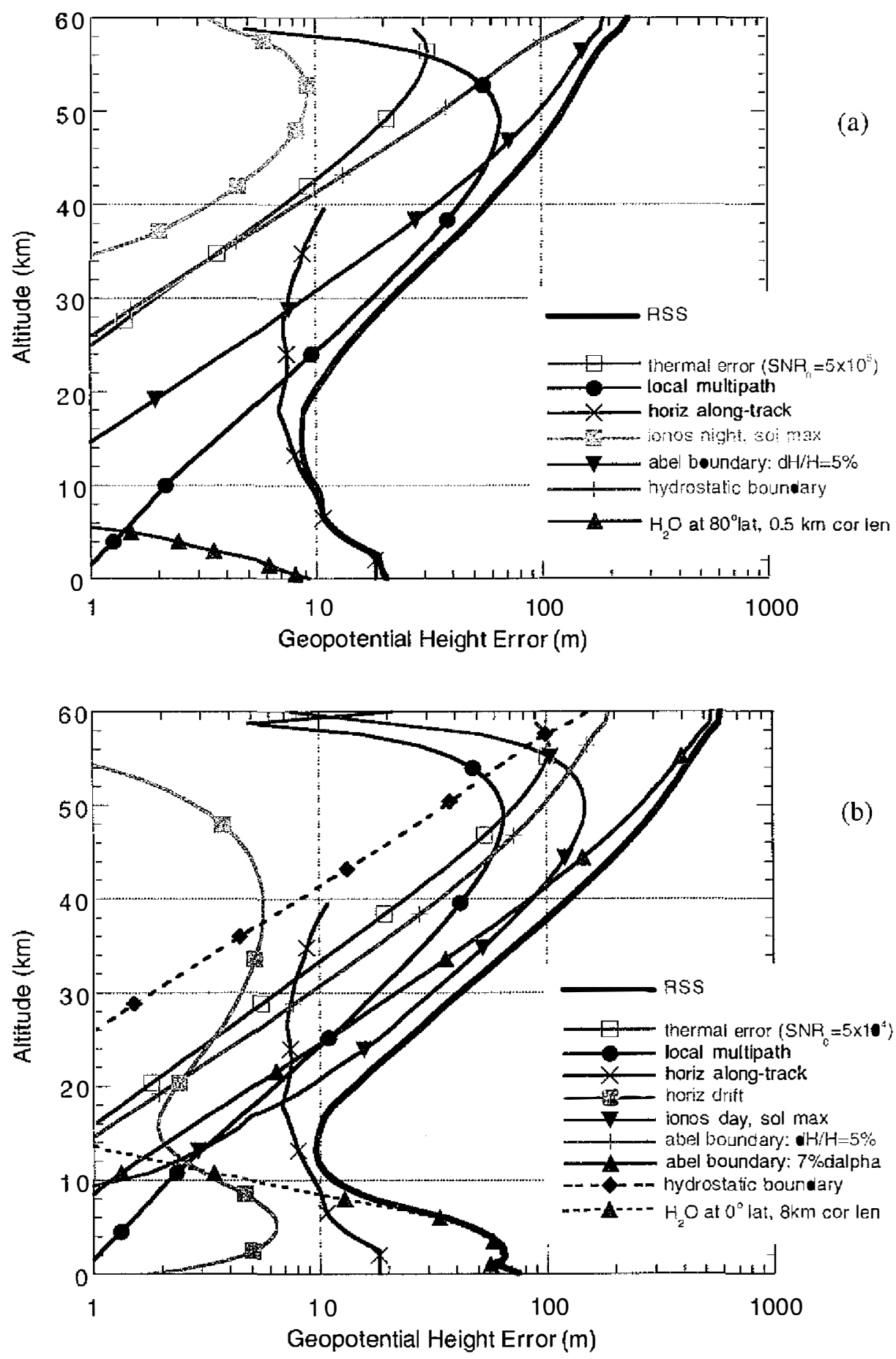

Fig. 16. Summary of geopotential height errors versus height from Kursinski et al., (1997). (a) and (b) conditions same as Figs. 15a and 15b. 


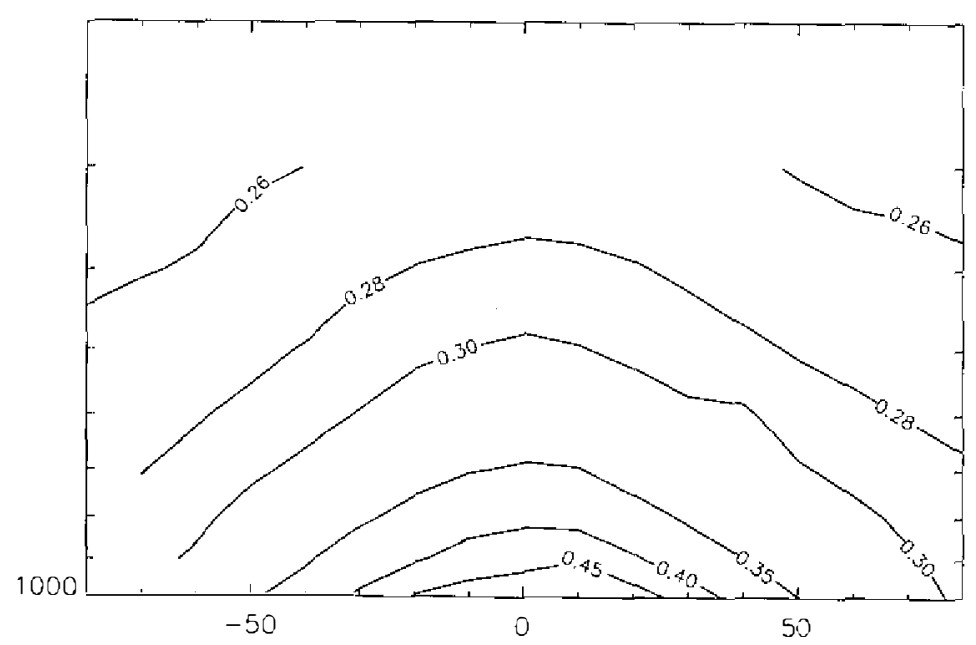

Fig. 17. Predicted RMS error in specific humidity (in $\mathrm{g} / \mathrm{kg}$ ) derived from GPS occultation refractivity observations and temperatures from global weather analyses accurate to $1.5 \mathrm{~K}$ based on annual mean climatology of Peixoto and Oort (1992). (Kursinski and Hajj (1999))

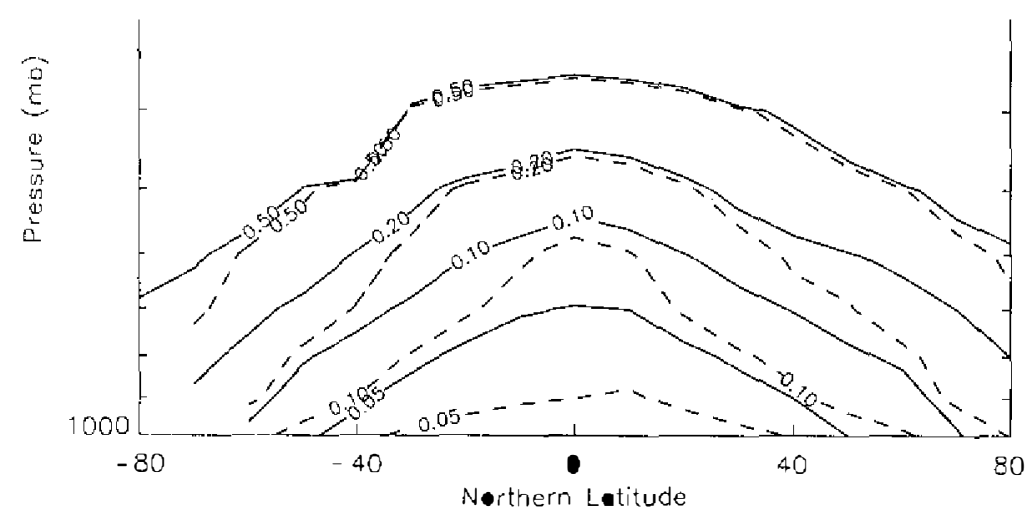

Fig. 18. Estimated fractional RMS error of individual derived water vapor profiles for annual mean conditions. Solid lines contain the temperature and pressure uncertainty contributions only. Dashed lines include the lower troposphere refractivity error as well. (Kursinski et al., 1995)

\section{INITIAL RESULTS}

Acquisition of the first GPS occultation data set commenced with the launch of the prototype GPS occultation mission, GPS/MET, in April, 1995 (Ware et al., 1996) from which temperature, geopotential and water vapor results have been derived. A summary of statistical comparisons is presented below. 


\subsection{Temperature}

Figure 19 shows a statistical comparison between GPS-derived temperatures and the 6 hour global analyses from the European Center for Medium-Range Weather Forecasts (ECMWF) interpolated to the occultation locations for several days in April and May 1995 (Kursinski et al., 1996). The lowest altitude of the data comparison is defined at the point where the temperature reaches $250 \mathrm{~K}$ in the troposphere, which avoids the water vapor ambiguity in interpreting the source of the retrieved refractivity. In the Northern Hemisphere where the analyses are presumed most accurate, mean differences are generally with $0.5 \mathrm{~K}$ and standard deviations are within $1 \mathrm{~K}$. The cause of the vertical structure in the tropical upper troposphere is unclear. The large scatter in the tropical lower stratosphere is due to waves that are resolved in the GPS data but not in the analyses. The Southern Hemisphere profiles were found to separate into two rather distinct groups shown in Fig. 20. While most of the GPS and ECMWF profiles were in close agreement, about one quarter of the profiles indicated the troposphere was too cold and the tropopause was too low in altitude in the analyses. All but one of the erroneous profiles was located in the southeastern Pacific, a region devoid of land and radiosondes.
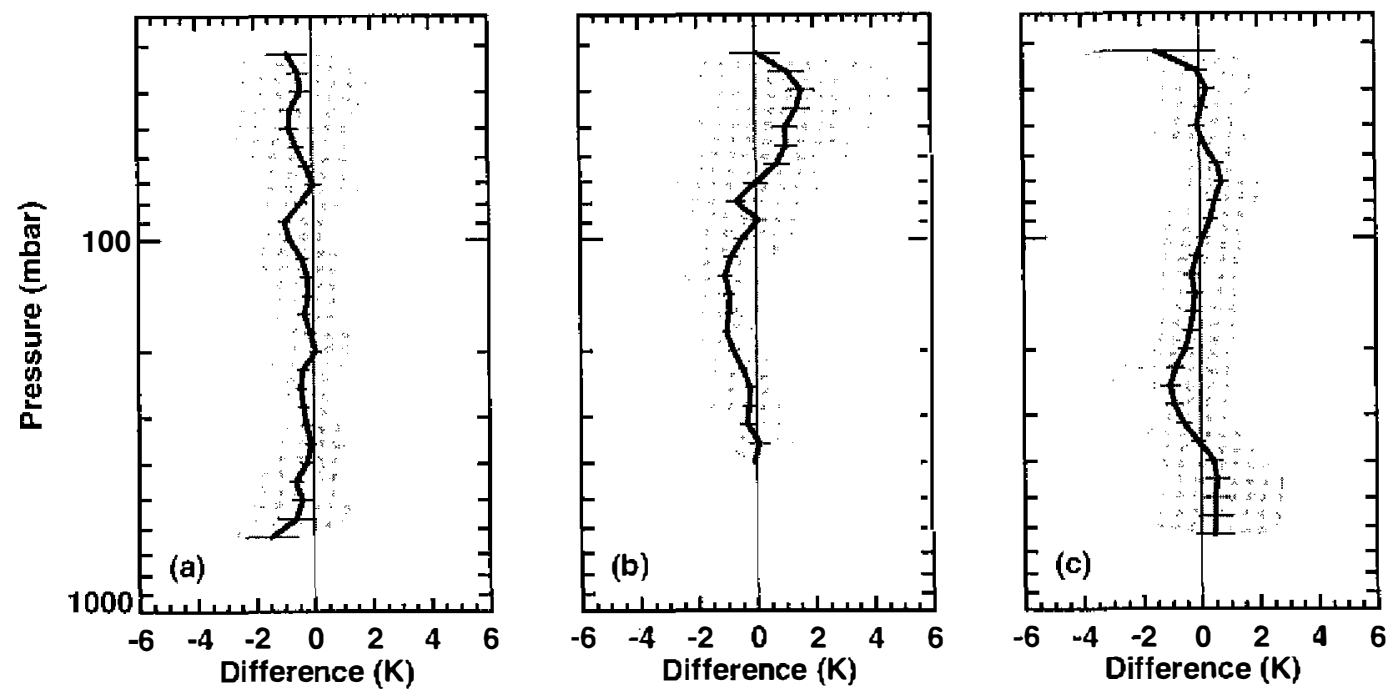

Fig. 19. Comparisons between the ECMWF analysis and temperature profiles retrieved from radio occultations on $4^{\text {th }}$ and $5^{\text {th }}$ May, 1995. The panels plot mean temperature differences (occultation-ECMWF) for (a) 34 profiles in the Northern Hemisphere $\left(>30^{\circ} \mathrm{N}\right)$, (b) 32 profiles in the tropics $\left(30^{\circ} \mathrm{S}\right.$ to $\left.30^{\circ} \mathrm{N}\right)$, and (c) 33 profiles in the Southern Hemisphere $\left(>30^{\circ} \mathrm{S}\right)$. The vertical curve represents mean temperature differences and the horizontal error bars depict the standard error in the mean. The shaded area is defined by the mean temperature differences plus or minus the standard deviation of the temperature differences about the mean. (Kursinski et al., 1996) 

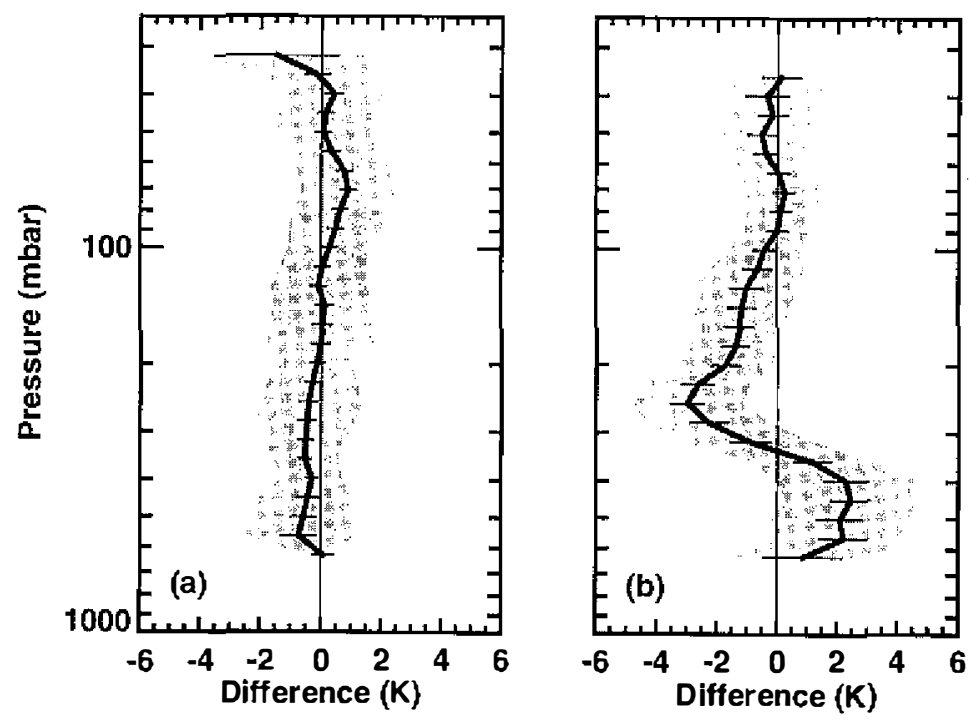

Fig. 20. Statistical comparison of the 33 southern hemisphere profiles from AprilMay 1995 period divided into two groups: (a) 25 profiles which differ little relative to the model and (b) 8 profiles with the largest deviations relative to the model. (Kursinski et al., 1996)

\subsection{Geopotential}

Figure 21 shows a statistical comparison between ECMWF and GPS-derived geopotential for June 21 to July 4, 1995, analogous to Fig. 19. Biases in the mean are 10 to $20 \mathrm{~m}$ in the Northern Hemisphere and almost zero in the tropics. Standard deviations in the Northern Hemisphere and tropics are $\sim 20 \mathrm{~m}$, roughly a factor of 2 larger than the predicted RMS errors in Fig. 16 indicating the prototype GPS/MET system is performing quite well in this regard. Reduced agreement in the Southern Hemisphere is consistent with sparse radiosonde data. The agreement in the lower stratosphere and approximately constant, positive bias in the troposphere imply the analyzed ECMWF surface pressures are too low in the Southern Hemisphere and the ECMWF temperatures are too warm near the tropopause. The low order spherical harmonic fit to the difference between ECMWF and GPS-derived geopotential height of the $300 \mathrm{mb}$ pressure surface in Fig. 22 shows the global structure of the differences. Some of the difference between the Northern and Southern Hemispheres is related to the diurnal cycle because of the orientation of the GPS/MET orbit during the June-July 1995 GPS/MET acquisition period. Systematic differences in behavior are particularly apparent in the southeastern Pacific where the analyses exhibit a systematically cold bias in the troposphere.

\subsection{Water Vapor}

Kursinski and Hajj (1999) derived some initial water vapor profiles from GPS/MET data using the interpolated ECMWF temperature field to isolate the water vapor contribution to 

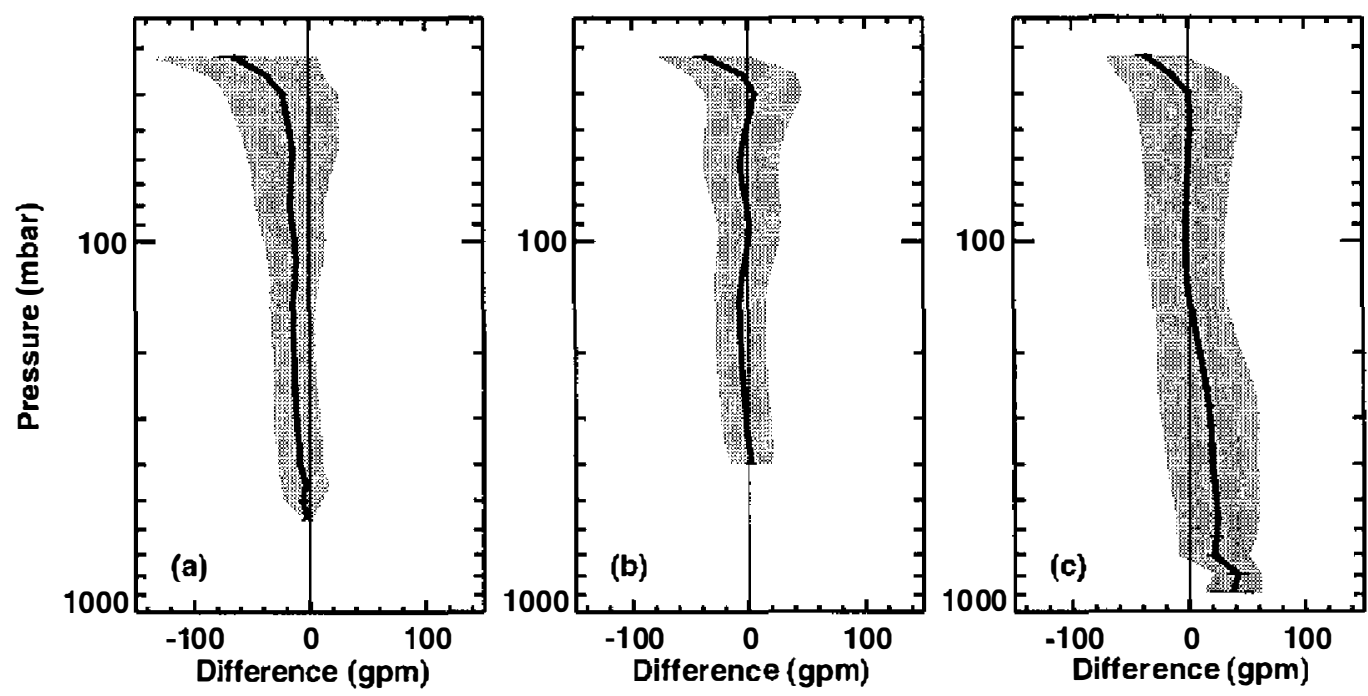

Fig. 21. Statistical comparison of geopotential height derived from GPS/MET data taken during the June-July period of 1995. (Leroy, 1997)
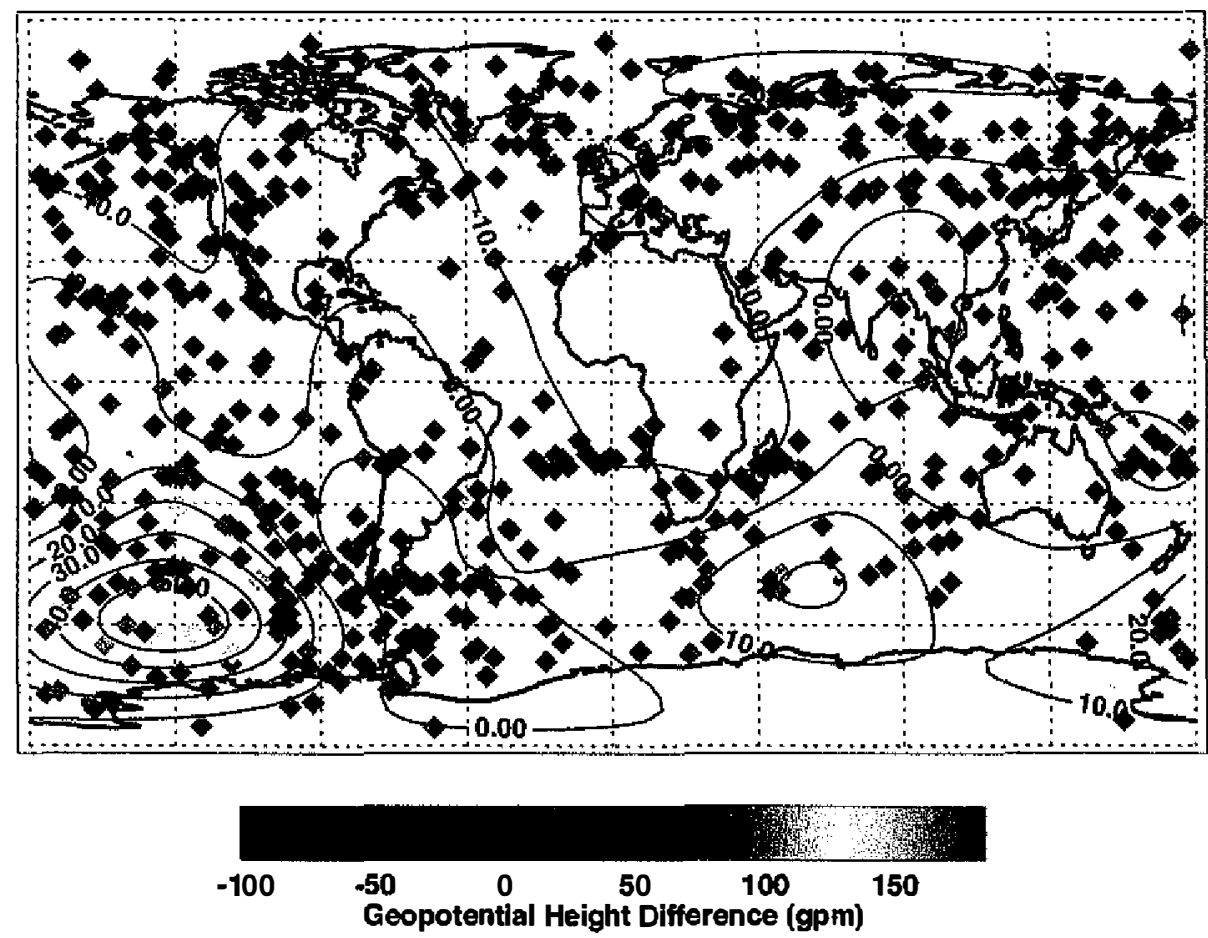

Fig. 22. Geopotential height difference at $300 \mathrm{mb}$ : Occultation - ECMWF June 21 - July 4, 1995. Contours are at $10 \mathrm{gpm}$ intervals. (Leroy, 1997). 
refractivity in (45). Derivation of water vapor begins when the GPS profile temperature reaches $230 \mathrm{~K}$ in the troposphere. Figure 23 shows the zonal mean specific humidity derived from the June 21 to July 4, 1995, span of GPS/MET data consisting of 800 occultations. Major features include the large summer-winter hemisphere contrast, peak humidities near the inter-tropical convergence zone (ITCZ) between $5^{\circ} \mathrm{N}$ and $10^{\circ} \mathrm{N}$, the dry descending branch of the Hadley circulation between $10^{\circ} \mathrm{S}$ and $20^{\circ} \mathrm{S}$ and high mean specific humidities near $30^{\circ} \mathrm{N}$ above $7 \mathrm{~km}$ associated with the southeast Asian monsoon. Also of note is the fact that many profiles extend to within $1 \mathrm{~km}$ of the surface indicating the ability of GPS to probe the lower troposphere.

Figure 24 shows the mean of the differences between the occultation-derived and ECMWF specific humidity fields. Agreement is $\sim 0.1 \mathrm{~g} / \mathrm{kg}$ in the upper regions. The GPS results suggest the atmosphere below the $500 \mathrm{mb}$ level is somewhat drier in general than the ECMWF humidity field except near $2 \mathrm{~km}$ altitude between the ITCZ and monsoon latitudes. ECMWF analyses are significantly more moist near the tradewind inversion between 0 and $30 \mathrm{~S}$. Examination of individual profiles reveals that the ECMWF analyses are smoothing out the vertical structure of the tradewind inversion and extending boundary layer air too high in altitude. The biases between ECMWF and occultation humidity fields may also indicate the tropical Hadley circulation is too weak in the analyses.

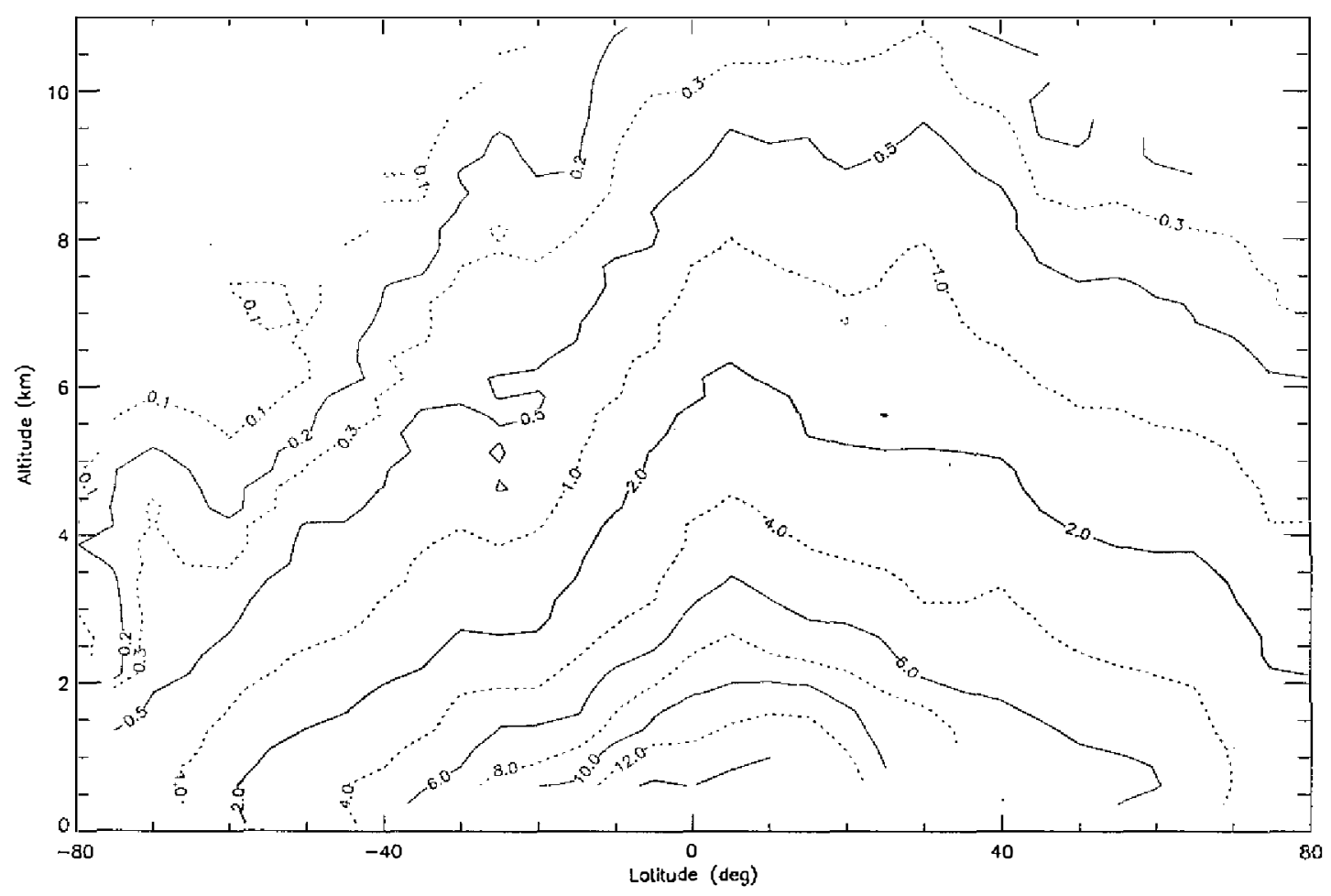

Fig. 23. Zonal mean specific humidity (in $\mathrm{g} / \mathrm{kg}$ ) derived from 800 GPS occultations acquired from June 21 to July 4, 1995 and ECMWF and NCEP temperature data. (Kursinski and Hajj, 1999). 


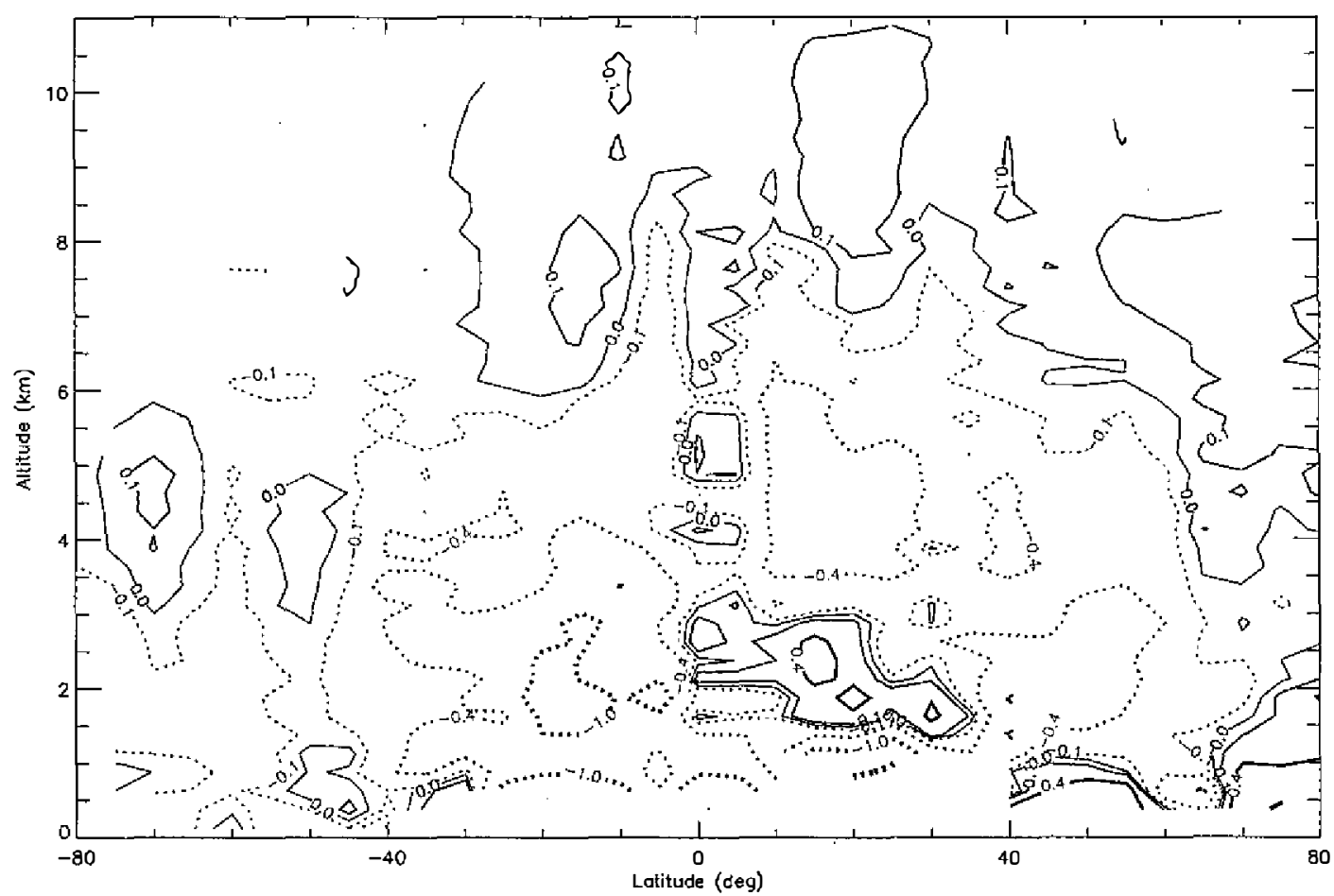

Fig. 24. Zonal mean specific humidity differences, GPS minus ECMWF, from June 21 to July 4,1995 . Contours are at $0, \pm 0.1, \pm 0.4, \pm 1, \pm 2 \mathrm{~g} / \mathrm{kg}$. Solid lines are positive, dotted lines are negative. (Kursinski and Hajj, 1999).

\section{SUMMARY AND CONCLUSIONS}

We have presented an overview of the GPS occultaion technique trying to touch upon the key issues and aspects of the basic retrieval technique. We began with a geometrical optics description of the signal propagation, and discussed why the occultation spherical symmetry approximation works well and justified the Abel transform pair in the process. We discussed the diffraction-limited resolution of the LEO observations and the backpropagation concept to reduce the effects of diffraction by backpropagating the signal through the intervening vacuum between the receiver and the atmosphere. We described how critical refraction limits the vertical range of observations and how one can interpolate the refraction results across intervals of critical refraction to recover refractivity within the regions. We discussed multipath in some detail, establishing the conditions under which multipath occurs, regions where multipath may occur and reduction in multipath with the backpropagation concept. We described the source of atmospheric refractivity, the interpretation of $\mathrm{N}$, and a least squares approach to combining the occultation observations with weather analyses to resolve the wet-dry ambiguity in tropospheric refractivity.

We discussed some of the key aspects of processing real data using amplitude information in estimating Doppler in a manner consistent with the diffraction resolution, removing 
clock errors, calibrating the effects of the ionosphere and handling the upper boundary condition of the Abel transform. We finished with a brief summary of predicted accuracy and a statistical comparison of initial GPS/MET results compared against the ECMWF global analyses demonstrating consistency comparable to that expected.

Acknowledgments The research described in this paper was carried out by the Jet Propulsion Laboratory, California Institute of Technology, and the University of Arizona under contract with the National Aeronautics and Space Administration.

\section{REFERENCES}

Ahmad, B., and G. L. Tyler, 1999: Systematic errors in atmospheric profiles obtained from Abelian inversion of radio occultation data: Effects of large scale horizontal gradients. J. Geophys. Res., 104, 3971-3992.

Atkins, P. W., 1983: Molecular Quantum Mechanics, 471 pp., Oxford University Press.

Augstein, E., H. Schmidt, and F. Ostapoff, 1974: The vertical structure of the atmospheric planetary boundary layer in undisturbed trade wind over the Atlantic Ocean. Boundary Layer Meteorol., 6, 129-150.

Bassiri, B., and G. A. Hajj, 1993: Higher-order ionospheric effects on the global positioning system observables and means of modeling them. Manuscr. Geod., 18, 280-289.

Born, M., and E. Wolf, 1980: Principles of Optics, 6th ed., Pergamon, Tarrytown, N. Y..

Chahine, M. T., D. J. McCleese, P. W.Rosenkranz, and D. H. Staelin, 1983: Interaction mechanisms within the atmosphere, in Manual of Remote Sensing, edited by R.N.Colwell, pp. 165-230, Am. Soc. of Photogramm., Falls Church, Va..

Eshleman, V. R., 1975: Jupiter's Atmosphere: Problems and Potential of Radio Occultation. Science, 189, 876-878.

Eyre, J. R., 1994: Assimilation of radio occultation measurements into a numberical weather prediction system. ECMWF Technical Memorandum No. 199, 34 pp.

Fjeldbo, G. F., V. R. Eshleman, and A. J. Kliore, 1971: The neutral atmosphere of Venus as studied with the Mariner V radio occultation experiments. Astron. J., 76, 123-140.

Gorbunov M. E., A. S. Gurvich, L. Bengtsson, 1996: Advanced algorithms of inversion of GPS/MET Satellite data and their application to reconstruction of temperature and humidity, Rep. 211, Max-Planck-Inst. fur Meteorol., Hamburg, F. R Germany, August.

Gorbunov, M. E. and S. V. Sokolovskiy, 1993: Remote Sensing of refractivity from space for global observations of atmospheric parameters. Max-Planck-Institute fur Meteorologie Report, No. 119, Hamburg, Germany, 58 pp.

Hajj, G. A., and E. R. Kursinski, 1991: Analysis of errors in the vertical temperature profiles recovered from GPS occultation observations. Eos Trans. AGU, 72 (44), Fall Meet. Suppl., 372.

Hajj, G. A., E. R. Kursinski, W. I. Bertiger, S. S. Leroy, T. Meehan, L. J. Romans, and J. T. Schofield, 1996: Initial results of GPS-LEO occultation measurements of Earth's atmosphere obtained with the GPS/MET experiment, Proc. IAG Symp. G1, GPS Trends in Precise Terrestrial, Airbome, and Spaceborne Applications, IUGG XXI General Assy., 
Boulder, CO, 2-14 July 1995, Springer-Verlag.

Hajj, G. A., R. Ibanez-Meier, E. R. Kursinski, and L. J. Romans, 1994: Imaging the ionosphere with the Global Positioning System.. Int. J. Imaging Sys. Tech., 5, 174-184.

Hajj, G. A., and L. J. Romans, 1998: Ionospheric electron density profiles obtained with the Global Positioning System: Results from the GPS/MET experiment. Radio Sci., 33, 175-190.

Hajj, G. A., E. R. Kursinski, L. J. Romans, W. I. Bertiger, and S. S. Leroy, 2000: A Technical Description of Atmospheric Sounding by GPS Occultations, submitted to J. Atmospheric and Oceanic Technology.

Hardy, K. R., G. A. Hajj, and E. R. Kursinski, 1994: Accuracies of atmospheric profiles obtained from GPS occultations. Int. J. Satell. Commun., 12, 463-473.

Haugstad, B. B., 1978: Effects of the inhomogeneous background on radiation propagating through turbulent planetary atmospheres. Radio Sci., 13, 435-440.

Healy, S. B. and J. R. Eyre, 2000: Retrieving temperature, water vapour and surface pressure information from refractive index profiles derived by radio occultation: a simulation study. Q. J. Royal Meteorol. Soc. in press.

Hinson, D. P., and J. A. Magalhaes, 1991: Equatorial waves in the stratosphere of Uranus. Icarus, 94, 64-91.

Karayel, E. T., and D.P. Hinson, 1997: Sub-Fresnel-scale vertical resolution in atmospheric profiles from radio occultation. Radio Sci., 32 (2), 411-423.

Kliore, A. J., D. L. Cain, G. Fjeldbo, B. L. Seidel, S. I. Rasool, 1974: Preliminary Results on the Atmospheres of Io and Jupiter from the Pioneer 10 S-band Occultation experiment. Science, 183, 323.

Kliore, A. J., G. Fjeldbo, B. L. Seidel, T. T. Sesplaukis, D. W. Sweetnam, P. M. Woiceshyn, 1975: The Atmosphere of Jupiter from the Pioneer 11 S-band Occultation experiment: Preliminary results. Science, 188, 474.

Kliore, A. J., P. M. Woiceshyn, and W. B. Hubbard, 1976: Temperature of the Atmosphere of Jupiter from Pioneer 10/11 Radio Occultations. Geophys. Res. Lett., 3, 113-116.

Kursinski, E. R., 1997: The GPS radio occultation concept: theoretical performance and initial results. Ph.D. thesis, Calif. Inst. of Technol., Pasadena.

Kursinski E. R., G. A. Hajj, and K.R. Hardy, 1993: Temperature or moisture profiles from radio occultation measurements, Proceedings of the Eighth Symposium on Meteorological Observations and Instrumentation. pp. J153-J158, Amer. Meteorol Soc., Anaheim CA, January 17-22.

Kursinski, E. R., G. A. Hajj, K. R. Hardy, L. J. Romans, and J. T. Schofield, 1995: Observing tropospheric water vapor by radio occultation using the Global Positioning System. Geophys. Res. Lett., 22, 2365-2368.

Kursinski, E. R, G. A. Hajj, et al., 1996: Initial results of radio occultation observations of Earth's atmosphere using the Global Positioning System. Science, 271, 1107-1110.

Kursinski, E. R., G. A. Hajj, K. R. Hardy, J. T. Schofield, and R. Linfield, 1997: Observing Earth's atmosphere with radio occultation measurements. J. Geophys. Res., 102, 2342923465.

Kursinski, E. R., and G. A. Hajj, 1999: An examination of water vapor derived from GPS 
occultation observations during June-July 1995. submitted to J. Geophys. Res..

Leroy, S. S., 1997: Measurement of geopotential heights by GPS radio occultation. J. Geophys. Res., 102, 6971-6986.

Liebe, H. J., 1989: MPM - an atmospheric millimeter-wave propoagation model.Int.J. Infrared Milli, 10, 631-650.

Lindal, G. F., J. R. Lyons, D. N. Sweetnam et al., 1987: The atmosphere of Uranus - Results of radio occultation measurements with Voyager 2.J. Geophys. Res., 92, 14987-15001.

Lindal, G. F., 1992: The Atmosphere of Neptune: An Analysis of Radio Occultation Data Acquired with Voyager. Astronomical J., 103, 967-982.

Marouf, E. A., G. L. Tyler, and P. A. Rosen, 1986: Profiling Saturn's Rings by Radio Occultation. Icarus, 68, 120-166.

Mortensen, M. D., R. S. Linfield, and E. R. Kursinski, 1999: Vertical resolution approaching $100 \mathrm{~m}$ for GPS occultations of the Earth's atmosphere. Radio Sci., 34, 1475-1484.

Owens, J. S., 1967: Optical refractive index of air: Dependence on pressure, temperature and composition. Appl. Opt., 6, 51-58.

Peixoto, J. P., and A. H. Oort, 1992: Physics of Climate, 520 pp., Am. Inst. Phys, New York.

Sovers O.J., and J. S. Border, 1990: Observation model and parameter partials for the JPL geodetic GPS modeling software "GPSOMC", JPL Publication 87-21, Rev. 2, June.

Spilker, J. J., 1980: GPS signal structure and performance characteristics. In Global Positioning System, Institute of Navigation, Washington, D.C., pp. 29-54.

Thayer, G. D., 1974: An improved equation for the radio refractive index of air. Radio Sci., 9 , 803-807.

Vorob'ev, V. V., and T. G. Krasil'nikova, 1994: Estimation of the accuracy of the atmospheric refractive index recovery from Doppler shift measurements at frequencies used in the NAVSTAR system. Phys. Atmos. Ocean, 29, 602-609.

Ware, R., et al., 1996: GPS sounding of the atmosphere from low earth orbit: Preliminary results. Bull. Am. Meteorol. Soc., 77(1), 19-40.

Wu, S. C., W. I. Bertiger, and J. T. Wu, 1990: Minimizing selective availability error on TOPEX GPS measurements, paper presented at the Conference on Astrodynamics, Am. Inst. of Aerosnaut. and Astronaut./Am. Astron. Soc., Portland, Oreg., Aug. 20-22.

Yunck, T. P., G. F. Lindal, and C. H. Liu, 1988: The role of GPS in precise earth observation, paper presented at the IEEE Position, Location and Navigation Symposium, Orlando, Fl., Nov. 29-Dec. 2.

Zou, X., F. Vandenberge, B. Wang, et al., 1999: A ray-tracing operator and its adjoint for the use of GPS/MET refraction angle measurements. J. Geophys. Res., 104, 22301-22318. 